\title{
BOUNDARY VALUE PROBLEMS AND OPTIMAL BOUNDARY CONTROL FOR THE NAVIER-STOKES SYSTEM: THE TWO-DIMENSIONAL CASE*
}

\author{
A. V. FURSIKOV ${ }^{\dagger}$, M. D. GUNZBURGER ${ }^{\ddagger}$, AND L. S. HOU $^{\S}$
}

\begin{abstract}
We study optimal boundary control problems for the two-dimensional Navier-Stokes equations in an unbounded domain. Control is effected through the Dirichlet boundary condition and is sought in a subset of the trace space of velocity fields with minimal regularity satisfying the energy estimates. An objective of interest is the drag functional. We first establish three important results for inhomogeneous boundary value problems for the Navier-Stokes equations; namely, we identify the trace space for the velocity fields possessing finite energy, we prove the existence of a solution for the Navier-Stokes equations with boundary data belonging to the trace space, and we identify the space in which the stress vector (along the boundary) of admissible solutions is well defined. Then, we prove the existence of an optimal solution over the control set. Finally, we justify the use of Lagrange multiplier principles, derive an optimality system of equations in the weak sense from which optimal states and controls may be determined, and prove that the optimality system of equations satisfies in appropriate senses a system of partial differential equations with boundary values.
\end{abstract}

Key words. optimal control, Navier-Stokes equations, boundary value problem, drag minimization

AMS subject classifications. 76D05, 49J20, 49K20, 35K50

PII. S0363012994273374

1. Introduction. Optimal control problems for fluid flows have been a subject of interest to experimenters and designers since at least the time of Prandtl. In more recent times, they have also become of substantial interest to mathematicians and computational scientists. For the steady state Navier-Stokes system, complete and systematic mathematical and numerical analyses of optimal control problems of different types (e.g., having Dirichlet, Neumann, and distributed controls and also finite-dimensional controls) were given in $[15,16,17,18]$. Mathematical treatments of optimal control problems for the time-dependent Navier-Stokes system were given in $[2],[6,7,8,9,10,11,12,13]$, [20], and [24, 25, 26, 27]. In [6], free convection problems with boundary heat flux controls were considered; the existence of optimal solutions was proved and necessary conditions that characterize optimal controls and states were derived. In $[11,12,13]$, the existence of optimal distributed controls was shown, an optimality system of equations was derived, and the question of the uniqueness

\footnotetext{
*Received by the editors August 24, 1994; accepted for publication (in revised form) February 24, 1997.

http://www.siam.org/journals/sicon/36-3/27337.html

${ }^{\dagger}$ Department of Mechanics and Mathematics, Moscow State University, Moscow 119899, Russia (fursikov@dial01.msu.ru). The research of this author was supported by Air Force Office of Scientific Research grant AFOSR-93-1-0280 while he was visiting Virginia Tech, and by Natural Science and Engineering Research Council of Canada grant OGP-0137436 while he was visiting York University.

$¥$ Interdisciplinary Center for Applied Mathematics, Virginia Polytechnic Institute and State University, Blacksburg, VA 24061-0531. Current address: Department of Mathematics, Iowa State University, Ames, IA 50011-2066 (gunzburg@iastate.edu). The research of this author was supported by Air Force Office of Scientific Research grant AFOSR-93-1-0280 and Office of Naval Research grant N00014-91-J-1493.

$\S$ Department of Mathematics and Statistics, York University, North York, ON M3J 1P3, Canada (hou@mathstat.yorku.ca). The research of this author was supported by the Natural Science and Engineering Research Council of Canada grant OGP-0137436.
} 
of optimal solutions was resolved. Distributed controls were also considered in [20]. Various optimal control problems involving both distributed and boundary controls were considered in [2], although detailed proofs were provided only for the case of distributed controls. In $[7,8,9,10]$ and $[24,25,26,27]$ extensive studies of optimal control problems were given for Dirichlet controls in a special case, namely, when the control is of the separation-of-variable type.

In this paper we consider general Dirichlet controls for the time-dependent, twodimensional Navier-Stokes system in the exterior of a bounded domain. Our eventual goal is to derive an optimality system from which optimal controls and states may be determined. A feature of the Dirichlet boundary control problem is as follows: one can derive an optimality system only in spaces of sufficiently smooth functions for which the nonlinear terms of the Navier-Stokes system are subordinate to the linear terms. (In the case of distributed control the situation is different; see [13].) In the two-dimensional case, the space of minimal smoothness possessing this property is the space of functions with "finite energy." Therefore, we first identify the space of boundary values which allows us to obtain finite energy solutions for the Navier-Stokes equations. Then, we prove the existence of an optimal solution in the finite energy space. Note that it would be easier to prove the solvability of an optimal control problem in a certain space of nonsmooth functions, but such a result is useless for the derivation of an optimality system. Finally, we use Lagrange multiplier techniques to derive a boundary value problem that the optimal states and control must satisfy. This boundary value problem is called the optimality system. We rigorously justify the boundary conditions for this system by means of techniques for elliptic boundary value problems in spaces of distributions and a theory, given below, about stress regularity for solutions of the Navier-Stokes equations with nonhomogeneous Dirichlet boundary conditions. In contrast to parabolic boundary value problems, it is here necessary to fulfill the compatibility conditions for boundary and initial values even in the case of nonsmooth solutions.

\section{Formulation of the problem.}

2.1. Derivation of the cost functional. We will formally derive the drag functional for flows surrounding a finite body. We consider the motion of an incompressible fluid in an unbounded domain that is described by the system

$$
\begin{gathered}
\rho \partial_{t} \mathbf{v}-\mu \Delta \mathbf{v}+\rho \mathbf{v} \cdot \nabla \mathbf{v}+\nabla p=\mathbf{0} \quad \text { and } \quad \nabla \cdot \mathbf{v}=0 \quad \text { in }(0, T) \times \Omega, \\
\left.\mathbf{v}\right|_{t=0}=\mathbf{v}_{0} \quad \text { for } \mathbf{x} \in \Omega,\left.\quad \mathbf{v}\right|_{\partial \Omega}=\mathbf{g} \quad \text { for } t \in(0, T),
\end{gathered}
$$

and

$$
\mathbf{v} \rightarrow \mathbf{v}_{\infty} \quad \text { as }|\mathbf{x}| \rightarrow \infty .
$$

Here, $\partial_{t}=\partial / \partial t, \Omega$ is the region exterior to a bounded body $B \subset \mathbb{R}^{2}$, and $\partial \Omega$ is its boundary. For simplicity we assume $\partial \Omega$ is of class $C^{\infty}$ and is a connected closed curve without self-intersections. Also, the density $\rho$ is a constant and $\mathbf{v}_{\infty}$ is a constant vector; the exact nature of the behavior at infinity will be discussed later. Later on we will add a condition on $p$ so that, for given $\mathbf{v}_{0}$, $\mathbf{g}$, and $\mathbf{v}_{\infty}$, the problem (2.1)-(2.3) has a unique solution. When $\mathbf{g}=\mathbf{0},(2.1)-(2.3)$ is the problem of a fluid moving around the body $B$ with uniform velocity $\mathbf{v}_{\infty}$ at infinity.

Denote by $\partial \Omega_{\epsilon}$ a smooth closed curve in a neighborhood of $\partial \Omega$, surrounding $\partial \Omega$ and lying inside $\Omega ; \Omega_{\epsilon}$ is the part of $\Omega$ bounded by $\partial \Omega_{\epsilon}$ and containing the point at 
infinity. Let $\mathcal{T}=-p \mathcal{I}+2 \mu \mathcal{D}$ be the stress tensor; here, $\mathcal{D}=\mathcal{D}(\mathbf{v})=\frac{1}{2}\left(\nabla \mathbf{v}+\nabla \mathbf{v}^{T}\right)$ is the rate of deformation tensor for the flow. Then, for $\mathbf{x} \in \partial \Omega_{\epsilon},(\mathcal{T} \mathbf{n})(t, \mathbf{x})$ is the force at a point $\mathbf{x}$ on $\partial \Omega_{\epsilon}$ which acts on the fluid in $\Omega_{\epsilon}$ at the time $t$; here, $\mathbf{n}$ denotes the unit normal to the curve $\partial \Omega_{\epsilon}$ which is outer with respect to $\Omega_{\epsilon}$. Thus,

$$
\int_{0}^{T} d t \int_{\partial \Omega_{\epsilon}}\left(\mathbf{v}-\mathbf{v}_{\infty}\right) \cdot(\mathcal{T} \mathbf{n}) d s
$$

is the work needed to overcome the drag exerted on the "body" $B_{\epsilon}=\mathbb{R}^{2} \backslash \Omega_{\epsilon}$ over the time interval $(0, T)$. After passing to the limit as $\epsilon \rightarrow 0$, we obtain the work needed to overcome the drag exerted on the given body $B=\mathbb{R}^{2} \backslash \Omega$ :

$$
\mathcal{W}=\int_{0}^{T} d t \int_{\partial \Omega}\left(\mathbf{v}-\mathbf{v}_{\infty}\right) \cdot(\mathcal{T} \mathbf{n}) d s .
$$

Using the definitions of $\mathcal{T}$ and $D$, and taking into account that $\mathbf{v}_{\infty}$ is a constant vector, we have that

$$
\begin{aligned}
\mathcal{W} & =\int_{0}^{T} d t \int_{\partial \Omega}\left(\mathbf{v}-\mathbf{v}_{\infty}\right) \cdot\left\{-p \mathbf{n}+\mu\left(\nabla \mathbf{v}+\nabla \mathbf{v}^{T}\right) \mathbf{n}\right\} d s \\
& =\int_{0}^{T} d t \int_{\partial \Omega}\left(\mathbf{v}-\mathbf{v}_{\infty}\right) \cdot\left\{-p \mathbf{n}+\mu\left(\nabla\left(\mathbf{v}-\mathbf{v}_{\infty}\right)+\nabla\left(\mathbf{v}-\mathbf{v}_{\infty}\right)^{T}\right) \mathbf{n}\right\} d s .
\end{aligned}
$$

Upon setting $\mathbf{w}=\mathbf{v}-\mathbf{v}_{\infty}$,

$$
\mathcal{W}=\int_{0}^{T} \int_{\partial \Omega} \mathbf{w} \cdot\{-p \mathbf{n}+2 \mu \mathcal{D}(\mathbf{w}) \mathbf{n}\} d s d t
$$

Let $\Omega_{R}=\Omega \cap\left\{\mathbf{x} \in \mathbb{R}^{2}:|\mathbf{x}|<R\right\}$ and $\Gamma_{R}=\partial \Omega_{R} \backslash \partial \Omega$ for sufficiently large $R$ such that the circle of radius $R$ centered at the origin contains $\Omega$. Using Green's formula we obtain

$$
\begin{array}{rl}
\int_{\Omega_{R}} & \mathbf{w} \cdot(\nabla \cdot \mathcal{D}(\mathbf{w})) d \mathbf{x} \\
\quad= & \int_{\partial \Omega} \mathbf{w} \cdot \mathcal{D}(\mathbf{w}) \mathbf{n} d s+\int_{\Gamma_{R}} \mathbf{w} \cdot \mathcal{D}(\mathbf{w}) \mathbf{n} d s-\int_{\Omega_{R}} \mathcal{D}(\mathbf{w}): \nabla \mathbf{w} d \mathbf{x},
\end{array}
$$

where $\partial_{j}=\partial / \partial x_{j}$; i.e., $\partial_{j}$ denotes the partial derivative with respect to the $j$ th coordinate, $\nabla \cdot \mathcal{S}$ for a two-tensor $\mathcal{S}=\left\{S_{i j}\right\}$ is defined as the vector $\left(\partial_{j} S_{1 j}, \partial_{j} S_{2 j}\right)^{T}$, and the colon notation denotes the scalar product operation on two two-tensors; i.e., for two-tensors $\mathcal{T}=\left\{T_{i j}\right\}$ and $\mathcal{S}=\left\{S_{i j}\right\}, \mathcal{T}: \mathcal{S}=T_{i j} S_{i j}$. Also, we have employed the convention that repeated indices imply summation. From (2.1) we have that $\mathbf{w}=\mathbf{v}-\mathbf{v}_{\infty}$ satisfies $\nabla \cdot \mathbf{w}=0$ so that (2.5) and the identity

$$
2 \nabla \cdot \mathcal{D}(\mathbf{w})=\Delta \mathbf{w}+\nabla(\nabla \cdot \mathbf{w})
$$

yield

$$
\int_{\partial \Omega} \mathbf{w} \cdot \mathcal{D}(\mathbf{w}) \mathbf{n} d s=\frac{1}{2} \int_{\Omega_{R}} \mathbf{w} \cdot \Delta \mathbf{w} d \mathbf{x}+\int_{\Omega_{R}} \mathcal{D}(\mathbf{w}): \nabla \mathbf{w} d \mathbf{x}-\int_{\Gamma_{R}} \mathbf{w} \cdot \mathcal{D}(\mathbf{w}) \mathbf{n} d s .
$$

The symmetry of the tensor $\mathcal{D}(\mathbf{w})$ yields

$$
\int_{\Omega_{R}} \mathcal{D}(\mathbf{w}): \nabla \mathbf{w} d \mathbf{x}=\int_{\Omega_{R}} \mathcal{D}(\mathbf{w}): \mathcal{D}(\mathbf{w}) d \mathbf{x}
$$


so that

$$
\int_{\partial \Omega} \mathbf{w} \cdot \mathcal{D}(\mathbf{w}) \mathbf{n} d s=\frac{1}{2} \int_{\Omega_{R}} \mathbf{w} \cdot \Delta \mathbf{w} d \mathbf{x}+\int_{\Omega_{R}} \mathcal{D}(\mathbf{w}): \mathcal{D}(\mathbf{w}) d \mathbf{x}-\int_{\Gamma_{R}} \mathbf{w} \cdot \mathcal{D}(\mathbf{w}) \mathbf{n} d s .
$$

Since $\mathcal{D}(\mathbf{w})=\mathcal{D}(\mathbf{v})$, the substitution of the last equation into (2.4) yields

$$
\begin{gathered}
\mathcal{W}=\int_{0}^{T} \int_{\Omega_{R}} \mathbf{w} \cdot(\mu \Delta \mathbf{w}-\nabla p) d \mathbf{x} d t+2 \mu \int_{0}^{T} \int_{\Omega_{R}} \mathcal{D}(\mathbf{v}): \mathcal{D}(\mathbf{v}) d \mathbf{x} d t \\
-2 \mu \int_{0}^{T} \int_{\Gamma_{R}} \mathbf{w} \cdot \mathcal{D}(\mathbf{w}) \mathbf{n} d s d t+\int_{0}^{T} \int_{\Gamma_{R}} p \mathbf{w} \cdot \mathbf{n} d s d t
\end{gathered}
$$

so that by taking the limit $R \rightarrow \infty$ we obtain

$$
\mathcal{W}=\int_{0}^{T} \int_{\Omega} \mathbf{w} \cdot(\mu \Delta \mathbf{w}-\nabla p) d \mathbf{x} d t+2 \mu \int_{0}^{T} \int_{\Omega} \mathcal{D}(\mathbf{v}): \mathcal{D}(\mathbf{v}) d \mathbf{x} d t .
$$

From (2.1) we have that $\mathbf{w}=\mathbf{v}-\mathbf{v}_{\infty}$ satisfies

$$
\rho \partial_{t} \mathbf{w}-\mu \Delta \mathbf{w}+\rho \mathbf{v} \cdot \nabla \mathbf{w}+\nabla p=\mathbf{0} .
$$

Combining (2.6) and the last equation yields

$$
\begin{array}{r}
\mathcal{W}=2 \mu \int_{0}^{T} d t \int_{\Omega} \mathcal{D}(\mathbf{v}): \mathcal{D}(\mathbf{v}) d \mathbf{x}+\frac{\rho}{2} \int_{0}^{T} d t \int_{\Omega} \partial_{t}|\mathbf{w}|^{2} d \mathbf{x} \\
+\rho \int_{0}^{T} d t \int_{\Omega}(\mathbf{v} \cdot \nabla \mathbf{w}) \cdot \mathbf{w} d \mathbf{x} \\
=2 \mu \int_{0}^{T} d t \int_{\Omega} \mathcal{D}(\mathbf{v}): \mathcal{D}(\mathbf{v}) d \mathbf{x}+\frac{\rho}{2} \int_{0}^{T} d t \int_{\partial \Omega}|\mathbf{w}|^{2} \mathbf{v} \cdot \mathbf{n} d s \\
+\frac{\rho}{2} \int_{\Omega}|\mathbf{w}(T, \mathbf{x})|^{2} d \mathbf{x}-\frac{\rho}{2} \int_{\Omega}|\mathbf{w}(0, \mathbf{x})|^{2} d \mathbf{x} .
\end{array}
$$

The integral $\frac{\rho}{2} \int_{\Omega}|\mathbf{w}(t, \mathbf{x})|^{2} d \mathbf{x}$ is the (finite) kinetic energy of the difference flow $\mathbf{w}=$ $\mathbf{v}-\mathbf{v}_{\infty}$. (Note that the kinetic energy of the flow $\frac{\rho}{2} \int_{\Omega}|\mathbf{v}(t, \mathbf{x})|^{2} d \mathbf{x}=\infty$.) We can rewrite $(2.7)$ as the energy equality

$$
\begin{aligned}
& \frac{\rho}{2} \int_{\Omega}|\mathbf{w}(0, \mathbf{x})|^{2} d \mathbf{x}+\int_{0}^{T} d t \int_{\partial \Omega} \mathbf{w} \cdot \mathcal{T} \mathbf{n} d s \\
& \quad=\frac{\rho}{2} \int_{\Omega}|\mathbf{w}(T, \mathbf{x})|^{2} d \mathbf{x}+2 \mu \int_{0}^{T} d t \int_{\Omega} \mathcal{D}(\mathbf{v}): \mathcal{D}(\mathbf{v}) d \mathbf{x}+\frac{\rho}{2} \int_{0}^{T} d t \int_{\partial \Omega}|\mathbf{w}|^{2} \mathbf{v} \cdot \mathbf{n} d s .
\end{aligned}
$$

This relation may be interpreted as follows: the initial kinetic energy of the difference flow plus the work due to drag is equal to the final, i.e., at $t=T$, value of the kinetic energy of the difference flow plus the energy dissipated due to friction plus the work done by the boundary control. Whenever the control is absent, i.e., whenever $\left.\mathbf{v}\right|_{\partial \Omega}=\mathbf{g}=\mathbf{0}$, the third integral on the right-hand side of the last equation vanishes. Since the initial kinetic energy of the difference flow is given, it is quite natural to take the right-hand side of the last equation as the cost functional (for convenience, we introduce a factor of one-half):

$$
\begin{aligned}
\mathcal{J}(\mathbf{w})=\frac{\rho}{4} \int_{\Omega}|\mathbf{w}(T, \mathbf{x})|^{2} d \mathbf{x}+ & \mu \int_{0}^{T} d t \int_{\Omega} \mathcal{D}(\mathbf{v}): \mathcal{D}(\mathbf{v}) d \mathbf{x} \\
& +\frac{\rho}{4} \int_{0}^{T} d t \int_{\partial \Omega}|\mathbf{w}|^{2} \mathbf{v} \cdot \mathbf{n} d s .
\end{aligned}
$$


2.2. Constraints on the control. For both physical and mathematical reasons, the size of the control should be constrained. Physically, one cannot realize controls of arbitrary size. Moreover, the cost of effecting control should be accounted for in the optimization process; e.g., one would not usually want to reduce the drag by a small amount if the cost of doing so is prohibitive. Limits on the size of the control are also needed in order to obtain a mathematically meaningful problem, e.g., to guarantee the existence of an optimal solution in a certain function class. Of course, the physical and mathematical needs for limiting the size of the control are not unrelated.

It is simpler to explain the ideas concerning constraining the size of the control in the steady state context in which we have the governing system

$$
\begin{aligned}
-\mu \Delta \mathbf{v}+\rho \mathbf{v} \cdot \nabla \mathbf{v}+\nabla p=\mathbf{0} \quad \text { and } \quad & \nabla \cdot \mathbf{v}=0 \quad \text { in } \Omega \\
\left.\mathbf{v}\right|_{\partial \Omega}=\mathbf{g}, \quad \text { and } \quad \mathbf{v} \rightarrow \mathbf{v}_{\infty} & \text { as }|\mathbf{x}| \rightarrow \infty
\end{aligned}
$$

and the cost functional

$$
\mathcal{J}_{s}(\mathbf{v})=\int_{\partial \Omega} \mathbf{w} \cdot \mathcal{T} \mathbf{n} d s=\mu \int_{\Omega} \mathcal{D}(\mathbf{v}): \mathcal{D}(\mathbf{v}) d \mathbf{x}+\frac{\rho}{4} \int_{\partial \Omega}|\mathbf{w}|^{2} \mathbf{v} \cdot \mathbf{n} d s,
$$

where $\mathbf{w}=\mathbf{v}-\mathbf{v}_{\infty}$, as noted previously. In all physically interesting situations one would want to minimize the drag functional (2.11). If there are no constraints on the control, i.e., on $\mathbf{v}$ along the boundary $\partial \Omega$, then it is easy to find a trivial control such that $\mathcal{J}_{s}(\mathbf{v})=0$. Indeed, if we take $\left.\mathbf{v}\right|_{\partial \Omega}=\mathbf{v}_{\infty}$, then the solution of (2.9)-(2.10) is given by $\mathbf{v}(\mathbf{x})=\mathbf{v}_{\infty}$ and $\nabla p=\mathbf{0}$, and then, clearly, $\mathcal{J}_{s}(\mathbf{v})=\mathcal{J}_{s}\left(\mathbf{v}_{\infty}\right)=0$. This implies that $\mathcal{J}_{s}(\mathbf{v})$ can possibly be negative, thereby the object occupying the region $B$ is being propelled rather than being dragged, exactly the opposite of what we want to study. Thus, constraining the control is not only natural from the physical point of view of conserving resources, but is necessary for the minimization problem to model properly the desired physical objectives. (Note that in the time-dependent case, we cannot choose $\mathbf{v}=\mathbf{v}_{\infty}$ due to the initial condition of (2.2); however, we still want to limit the size of the control for the same reasons as in the steady state case.)

There are two common ways of constraining the control. The first one is to impose an explicit bound on the control. In the steady state case, we can impose

$$
\int_{\partial \Omega}|\mathbf{v}|^{k} d s \leq M \text { for some } k \geq 3
$$

or

$$
|\mathbf{v}(\mathbf{x})| \leq M \quad \forall \mathbf{x} \in \partial \Omega,
$$

where $M$ is a prescribed positive constant. The constraint (2.12) allows the control to concentrate on small portions of the boundary and is therefore more useful in providing information about the locations where the control is most effective. Such information will be helpful in the study of "local controls," i.e., the application of control at a number of chosen locations on the boundary. (We will study local control problems elsewhere.) For this reason we will not pursue constraints of the type (2.13) any further in this paper. The second way of constraining the control is to add some norm of the control to the cost functional; e.g., instead of (2.11), we consider the functional

$$
\mu \int_{\Omega} \mathcal{D}(\mathbf{v}): \mathcal{D}(\mathbf{v}) d \mathbf{x}+\frac{\rho}{4} \int_{\partial \Omega}|\mathbf{w}|^{2} \mathbf{v} \cdot \mathbf{n} d s+\rho N \int_{\partial \Omega}|\mathbf{v}|^{k} d s
$$


for some $k \geq 3$ and $N>0$. If $k=3$, we will need $N>\frac{1}{4}$. In both ways of constraining the control, one can use different norms to measure the control. The physical problem does not tell us which norm to use, although desirable physical properties, e.g., having no sharp peaks in the control along the boundary, should influence the choice. The choice of norm is also influenced by the need to establish the well-posedness of the problem, e.g., the existence of an optimal solution in some function class, the regularity of the optimal solution, etc. For example, the constraints on the value of $k$ are motivated by the need to have the cost of control, i.e., the last term in (2.14), dominate (in an appropriate sense that will be made clear later in this paper) the second term.

Our interest in this paper is in the time-dependent problem, and we now discuss how we can choose a convenient norm for measuring the control. Our starting point is the requirement that solutions of the Navier-Stokes system have energy estimates that will be needed later in this paper in studying the optimal control problems, and particularly in the derivation of the optimality system of equations. The minimum level of smoothness for the velocity field $\mathbf{v}$ at which the energy estimates are valid is $\mathbf{v}-$ $\mathbf{v}_{\infty} \in L^{2}\left(0, T ; \mathbf{H}^{1}(\Omega)\right)$ and $\partial_{t} \mathbf{v} \in L^{2}\left(0, T ; \mathbf{H}^{-1}(\Omega)\right)$. Note that these inclusions imply a certain behavior at infinity. (The Sobolev space notation used here is established in section 3.1.) The boundary control should belong to a subset of the trace space on $\partial \Omega$ of the space for the vector field $\mathbf{v}$. The norm on the trace space will be shown to be

$$
\begin{aligned}
& \|\mathbf{v} \cdot \mathbf{n}\|_{L^{2}\left(0, T ; H^{1 / 2}(\partial \Omega)\right)}+\|\mathbf{v} \cdot \mathbf{n}\|_{H^{3 / 4}\left(0, T ; H^{-1}(\partial \Omega)\right)} \\
& +\|\mathbf{v} \cdot \boldsymbol{\tau}\|_{L^{2}\left(0, T ; H^{1 / 2}(\partial \Omega)\right)}+\|\mathbf{v} \cdot \boldsymbol{\tau}\|_{H^{1 / 4}\left(0, T ; L^{2}(\partial \Omega)\right)},
\end{aligned}
$$

where $\boldsymbol{\tau}$ denotes the counterclockwise unit tangent vector to $\partial \Omega$. Naturally, the control should be measured in a norm that is not weaker than the norm for the desired trace space. For computational convenience, we will strengthen the fractional time derivative to the first derivative $\partial_{t}$ in the functional. Also, the particular form of the functional (2.8), i.e., the term $\int_{0}^{T} \int_{\Omega} \mathcal{D}(\mathbf{v}): \mathcal{D}(\mathbf{v}) d \mathbf{x} d t$, implies that in order for $\mathbf{v}$ to belong to the desired trace space, it is sufficient to use the norm

$$
\int_{0}^{T} \int_{\partial \Omega}\left|\partial_{t} \mathbf{v}\right|^{2} d s d t
$$

for the controls. Also, as in (2.12), we have to include the constraints connected with the term $\int_{0}^{T} \int_{\partial \Omega}|\mathbf{v}|^{k} d s d t$ for some $k \geq 3$.

Hence, the two approaches of constraining the control in the time-dependent case can now be described as follows. The first approach, i.e., imposing an explicit bound on the control, requires that, for some constant $M>0$,

$$
\int_{0}^{T} \int_{\partial \Omega}|\mathbf{v}|^{k} d s d t+\int_{0}^{T} \int_{\partial \Omega}\left|\partial_{t} \mathbf{v}\right|^{2} d s d t \leq M
$$

where $k \geq 3$. The second approach, i.e., adding a norm of the control to the functional, uses the functional

$$
\begin{aligned}
\mathcal{J}_{N}(\mathbf{v})= & \mu \int_{0}^{T} \int_{\Omega} \mathcal{D}(\mathbf{v}): \mathcal{D}(\mathbf{v}) d \mathbf{x} d t+\frac{\rho}{4} \int_{0}^{T} \int_{\partial \Omega}|\mathbf{w}|^{2} \mathbf{v} \cdot \mathbf{n} d s d t \\
& +\frac{\rho}{4} \int_{\Omega}|\mathbf{w}(T, \mathbf{x})|^{2} d \mathbf{x}+\rho N\left(\int_{0}^{T} \int_{\partial \Omega}|\mathbf{v}|^{k} d s d t+\int_{0}^{T} \int_{\partial \Omega}\left|\partial_{t} \mathbf{v}\right|^{2} d s d t\right),
\end{aligned}
$$

where $\mathbf{w}=\mathbf{v}-\mathbf{v}_{\infty}, k \geq 3$, and $N>0\left(N>\frac{1}{4}\right.$ if $\left.k=3\right)$. 
3. Precise statement of extremal problems. We will use the standard notations for the Lebesgue function space $L^{r}(\Omega)$ and the Sobolev spaces $W^{m, r}(\Omega), H^{m}(\Omega)$, $W^{l, s}(\partial \Omega)$, and $H^{l}(\partial \Omega)$ for real numbers $r, m, l, s$, where $m, l$ are the smoothness indices and $r, s$ are the integrability indices. Also, $H^{m}(\Omega)=W^{m, 2}(\Omega)$ and $H^{l}(\partial \Omega)=W^{l, 2}(\partial \Omega)$. For $m \geq 0$, we introduce the subspaces of the Sobolev spaces $W^{m, r}(\Omega)$ :

$$
W_{0}^{m, r}(\Omega)=\text { the closure of } C_{0}^{\infty}(\Omega) \text { in } W^{m, r}(\Omega)
$$

and the dual spaces

$$
W^{-m, r}(\Omega)=\left(W_{0}^{m, r^{\prime}}(\Omega)\right)^{*}, \quad \text { where } \frac{1}{r}+\frac{1}{r^{\prime}}=1,1<r, r^{\prime}<\infty .
$$

Also, $H_{0}^{m}(\Omega)=W_{0}^{m, 2}(\Omega)$ and $H^{-m}(\Omega)=W^{-m, 2}(\Omega)$. The vector counterparts of these spaces are denoted by $\mathbf{L}^{r}(\Omega), \mathbf{W}^{m, r}(\Omega), \mathbf{H}^{m}(\Omega), \mathbf{W}^{l, s}(\partial \Omega), \mathbf{H}^{l}(\partial \Omega), \mathbf{W}_{0}^{m, r}(\Omega)$, and $\mathbf{H}_{0}^{m}(\Omega)$. For details, see [1] and [14]. We will also use the solenoidal spaces

$$
\mathbf{V}^{m}(\Omega)=\left\{\mathbf{u} \in \mathbf{H}^{m}(\Omega): \nabla \cdot \mathbf{u}=0, \int_{\partial \Omega} \mathbf{u} \cdot \mathbf{n} d s=0\right\} \quad \text { for } m \geq 0
$$

and

$$
\mathbf{V}_{0}^{m}(\Omega)=\text { the closure of } \mathbf{C}_{0}^{\infty}(\Omega) \cap \mathbf{V}^{0}(\Omega) \text { in the } \mathbf{H}^{m}(\Omega) \text {-norm } \quad \text { for } m \geq 0,
$$

where when $m=0, \int_{\partial \Omega} \mathbf{u} \cdot \mathbf{n} d s$ is understood as the $H^{-1 / 2}(\partial \Omega)-H^{1 / 2}(\partial \Omega)$ duality pairing between the function $(\mathbf{u} \cdot \mathbf{n}) \in H^{-1 / 2}(\partial \Omega)$ and the constant scalar function $1 \in H^{1 / 2}(\partial \Omega)$. Note that in the definition of $\mathbf{V}^{m}(\Omega)$ ( $\Omega$ being unbounded), the condition $\int_{\partial \Omega} \mathbf{u} \cdot \mathbf{n} d s=0$ does not follow from $\operatorname{div} \mathbf{u}=0$ unless some additional assumptions are made on $\mathbf{u}$ at $\infty$. Note also that for simplicity, we have assumed $\partial \Omega$ is a connected curve; otherwise, we need to require $\int_{\Gamma_{i}} \mathbf{u} \cdot \mathbf{n} d s=0$ on each connected component $\Gamma_{i}$ of $\partial \Omega$. Identifying $\left(\mathbf{V}^{0}(\Omega)\right)^{*}$ with $\mathbf{V}^{0}(\Omega)$ we introduce the dual spaces

$$
\mathbf{V}^{-m}(\Omega)=\left[\mathbf{V}_{0}^{m}(\Omega)\right]^{*} \quad \text { for } m \geq 1 \text {. }
$$

The norms on $\mathbf{V}^{m}(\Omega)$ and $\mathbf{V}_{0}^{m}(\Omega)$ are chosen to be that of $\mathbf{H}^{m}(\Omega)$. We also introduce the temporal-spatial function space, defined on $Q=\mathbb{R} \times \Omega$,

$$
\mathcal{H}^{(s)}(Q)=\left\{f \in L^{2}\left(\mathbb{R} ; H^{s}(\Omega)\right): \partial_{t} f \in L^{2}\left(\mathbb{R} ; H^{s-2}(\Omega)\right)\right\}
$$

with norm

$$
\|f\|_{\mathcal{H}^{(s)}(Q)}^{2}=\|f\|_{L^{2}\left(\mathbb{R} ; H^{s}(\Omega)\right)}^{2}+\left\|\partial_{t} f\right\|_{L^{2}\left(\mathbb{R} ; H^{s-2}(\Omega)\right)}^{2},
$$

and the corresponding solenoidal function space

$$
\mathcal{V}^{(s)}(Q)=\left\{\mathbf{v} \in L^{2}\left(\mathbb{R} ; \mathbf{V}^{s}(\Omega)\right): \partial_{t} \mathbf{v} \in L^{2}\left(\mathbb{R} ; \mathbf{V}^{s-2}(\Omega)\right)\right\}
$$

with norm

$$
\|\mathbf{v}\|_{\mathcal{V}^{(s)}(Q)}^{2}=\|\mathbf{v}\|_{L^{2}\left(\mathbb{R} ; \mathbf{V}^{s}(\Omega)\right)}^{2}+\left\|\partial_{t} \mathbf{v}\right\|_{L^{2}\left(\mathbb{R} ; \mathbf{V}^{s-2}(\Omega)\right)}^{2}
$$

Analogously, we may define the function spaces $\mathcal{H}^{(s)}\left(Q_{T}\right)$ and $\mathcal{V}^{(s)}\left(Q_{T}\right)$ defined on $Q_{T}=(0, T) \times \Omega$. 
With the help of the spaces defined above, we may define the solution for the Navier-Stokes equations (2.1)-(2.3). We first quote a useful lemma.

LEMMA 3.1. The space $\mathcal{V}^{(1)}\left(Q_{T}\right)$ is continuously imbedded into $C\left([0, T] ; \mathbf{V}^{0}(\Omega)\right)$.

Proof. Solving in $Q_{T}$ the equations $\partial F(t, x) / \partial x_{2}=u_{1}, \partial F(t, x) / \partial x_{1}=-u_{2}$, and $\int_{\Omega} F(t, x) d x=0$ almost everywhere (a.e.) $t \in(0, T)$ for an arbitrary $u=\left(u_{1}, u_{2}\right) \in$ $\mathcal{V}^{(1)}\left(Q_{T}\right)$, we reduce the proof of the lemma to a proof of the continuity of the embedding $\mathcal{H}^{(2)}\left(Q_{T}\right) \subset C\left([0, T] ; H^{1}(\Omega)\right)$. The last assertion is proved in [4] or [21]. (For an alternate proof, see [5] or [28].)

Below, for the sake of simplicity, we set the constant density $\rho=1$ or, more precisely, we introduce nondimensionalized variables so that now $\mu$ is the inverse of the Reynolds number.

DEFINITION 3.2. $\mathbf{v}$ is said to be a solution of (2.1)-(2.3) if $\mathbf{v}=\mathbf{w}+\mathbf{v}_{\infty}$, where $\mathbf{w} \in \mathcal{V}^{(1)}\left(Q_{T}\right)$ satisfies

$$
\begin{aligned}
& \left\langle\partial_{t} \mathbf{w}(t), \mathbf{z}\right\rangle+2 \mu \int_{\Omega} \mathcal{D}(\mathbf{w}(t)): \mathcal{D}(\mathbf{z}) d \mathbf{x}+\int_{\Omega}(\mathbf{w}(t) \cdot \nabla) \mathbf{w}(t) \cdot \mathbf{z} d \mathbf{x} \\
& +\int_{\Omega}\left(\mathbf{v}_{\infty} \cdot \nabla\right) \mathbf{w}(t) \cdot \mathbf{z} d \mathbf{x}=0 \quad \forall \mathbf{z} \in \mathbf{V}_{0}^{1}(\Omega), \text { a.e. } t \in(0, T), \\
& \mathbf{w}=\mathbf{b} \equiv \mathbf{g}-\mathbf{v}_{\infty} \quad \text { in } L^{2}\left(0, T ; \mathbf{H}^{1 / 2}(\partial \Omega)\right),
\end{aligned}
$$

and

$$
\left.\mathbf{w}\right|_{t=0}=\mathbf{w}_{0} \equiv \mathbf{v}_{0}-\mathbf{v}_{\infty} \quad \text { in } \mathbf{V}^{0}(\Omega) .
$$

Note that the initial condition in Definition 3.2 makes sense because of Lemma 3.1. Here and elsewhere in this paper, $\langle\cdot, \cdot\rangle$ denotes the duality pairing between a Banach space and its dual space; the underlying Banach space may vary depending on the context. In particular, $\langle\cdot, \cdot\rangle$ in $(3.1)$ denotes the duality pairing between $\mathbf{V}^{-1}(\Omega)$ and $\mathbf{V}_{0}^{1}(\Omega)$. Also, note that we have used the identity

$$
2 \int_{\Omega} \mathcal{D}(\mathbf{w}): \mathcal{D}(\mathbf{z}) d \mathbf{x}=\int_{\Omega} \nabla \mathbf{w}: \nabla \mathbf{z} d \mathbf{x} \quad \forall \mathbf{w} \in \mathbf{V}^{1}(\Omega), \mathbf{z} \in \mathbf{H}_{0}^{1}(\Omega) .
$$

The extremal problems we study involve the objective of drag minimization. Based on the two ways of constraining the control, we have the two functionals (2.8) or (2.16) so that we state two extremal problems. It is more convenient to use the variable $\mathbf{w}=\mathbf{v}-\mathbf{v}_{\infty}$. Also, we will simply use $\left.\mathbf{w}\right|_{\partial \Omega}$ to denote the Dirichlet control and, thus, we will not introduce a separate notation to denote the control variable and the boundary condition (3.2) will not be explicitly imposed as a constraint. Extremal solutions are sought in the space

$$
\begin{aligned}
Y=\left\{\mathbf{w} \in \mathcal{V}^{(1)}\left(Q_{T}\right)\right. & :\left.\left(\partial_{t} \mathbf{w}\right)\right|_{\partial \Omega} \in L^{2}\left(0, T ; \mathbf{L}^{2}(\partial \Omega)\right), \\
& \left.\int_{\partial \Omega} \partial_{t} \mathbf{w} \cdot \mathbf{n} d s=0,\left.\quad \mathbf{w}\right|_{\partial \Omega} \in \mathbf{L}^{k}((0, T) \times \partial \Omega)\right\}
\end{aligned}
$$

equipped with the norm

$$
\|\mathbf{w}\|_{Y}=\|\mathbf{w}\|_{\mathcal{V}^{(1)}\left(Q_{T}\right)}+\left\|\partial_{t} \mathbf{w}\right\|_{L^{2}\left(0, T ; \mathbf{L}^{2}(\partial \Omega)\right)}+\|\mathbf{w}\|_{\mathbf{L}^{k}((0, T) \times \partial \Omega)},
$$

where $k \geq 3$ and $\mathbf{n}$ is the outward normal on $\partial \Omega$. 
We also introduce the space

$$
\mathbf{W}=\left\{\mathbf{w} \in \mathbf{V}^{0}(\Omega):\left.(\mathbf{w} \cdot \mathbf{n})\right|_{\partial \Omega} \in H^{1 / 4}(\partial \Omega) \cap L^{1+k / 2}(\partial \Omega)\right\} .
$$

Since the trace $\left.\gamma_{n, \partial \Omega}(\mathbf{w}) \equiv(\mathbf{w} \cdot \mathbf{n})\right|_{\partial \Omega}$ is well defined and belongs to $H^{-1 / 2}(\partial \Omega)$ (see $[28])$, the definition of $\mathbf{W}$ makes sense. Note that the restriction operator

$$
\gamma_{0}: Y \rightarrow \mathbf{W}
$$

defined by $\gamma_{0} \mathbf{w}=\left.\mathbf{w}\right|_{t=0}$ is continuous. Indeed, we denote

$$
\begin{aligned}
& Y_{\delta}=\left\{\mathbf{w} \in L^{2}\left(0, T ; \mathbf{H}^{1 / 2}(\partial \Omega)\right) \cap \mathbf{L}^{k}((0, T) \times \partial \Omega):\right. \\
& \left.\partial_{t} \mathbf{w} \in \mathbf{L}^{2}((0, T) \times \partial \Omega), \int_{\partial \Omega} \partial_{t} \mathbf{w} d s d s=0\right\}
\end{aligned}
$$

Then, since the trace operators $\gamma_{n, \partial \Omega}: Y \rightarrow Y_{\delta}$ and $\gamma_{0}: Y_{\delta} \rightarrow H^{1 / 4}(\partial \Omega) \cap L^{1+k / 2}(\partial \Omega)$ are continuous, the restriction $\gamma_{0} \mathbf{w}$ for an arbitrary $\mathbf{w} \in Y$ possesses the property

$$
\gamma_{n, \partial \Omega}\left(\gamma_{0} \mathbf{w}\right)=\gamma_{0}\left(\gamma_{n, \partial \Omega} \mathbf{w}\right) \in H^{1 / 4}(\partial \Omega) \cap L^{1+k / 2}(\partial \Omega)
$$

This proves that the imbedding $\gamma_{0} Y \subset \mathbf{W}$ is continuous. We intend to look for an extremal solution in the space $Y$. Thus, we are compelled to replace the initial condition in Definition 3.2 by

$$
\left.\mathbf{w}\right|_{t=0}=\mathbf{w}_{0} \equiv \mathbf{v}_{0}-\mathbf{v}_{\infty} \in \mathbf{W}
$$

Problem I. Suppose that $\mathbf{w}_{0} \equiv \mathbf{v}_{0}-\mathbf{v}_{\infty} \in \mathbf{W}$. Seek a $\mathbf{w} \in Y$ such that the functional

$$
\begin{aligned}
\mathcal{J}_{N}(\mathbf{w}) & =\mu \int_{0}^{T} \int_{\Omega} \mathcal{D}(\mathbf{w}): \mathcal{D}(\mathbf{w}) d \mathbf{x} d t \\
+ & \frac{1}{4} \int_{0}^{T} \int_{\partial \Omega}|\mathbf{w}|^{2}\left(\mathbf{w}+\mathbf{v}_{\infty}\right) \cdot \mathbf{n} d s d t+\frac{1}{4} \int_{\Omega}|\mathbf{w}(T, \mathbf{x})|^{2} d \mathbf{x} \\
& +N \int_{0}^{T} \int_{\partial \Omega}\left(\left|\mathbf{w}+\mathbf{v}_{\infty}\right|^{k}+\left|\partial_{t} \mathbf{w}\right|^{2}\right) d s d t
\end{aligned}
$$

is minimized subject to the constraints (3.1) and (3.3), where $k \geq 3$ and $N>0$ with $N>\frac{1}{4}$ if $k=3$.

Problem II. Suppose that $\mathbf{w}_{0} \equiv \mathbf{v}_{0}-\mathbf{v}_{\infty} \in \mathbf{W}$. Seek a $\mathbf{w} \in Y$ such that the functional

$$
\begin{array}{r}
\mathcal{J}(\mathbf{w})=\mu \int_{0}^{T} \int_{\Omega} \mathcal{D}(\mathbf{w}): \mathcal{D}(\mathbf{w}) d \mathbf{x} d t+\frac{1}{4} \int_{0}^{T} \int_{\partial \Omega}|\mathbf{w}|^{2}\left(\mathbf{w}+\mathbf{v}_{\infty}\right) \cdot \mathbf{n} d s d t \\
+\frac{1}{4} \int_{\Omega}|\mathbf{w}(T, \mathbf{x})|^{2} d \mathbf{x}
\end{array}
$$

is minimized subject to the constraints $(3.1),(3.3)$, and

$$
\int_{0}^{T} \int_{\partial \Omega}\left(\left|\mathbf{w}+\mathbf{v}_{\infty}\right|^{k}+\left|\partial_{t} \mathbf{w}\right|^{2}\right) d s d t \leq M
$$

where $k \geq 3$ and $M>0$. 
Note that Lemma 3.1 ensures that the functionals (3.4) and (3.5) are well defined on $Y$. We now give definitions for an admissible element and for a solution of Problem I or II.

DeFinition 3.3. An element $\mathbf{w} \in Y$ is called admissible if it satisfies (3.1) and (3.3) in the case of Problem I and satisfies (3.1), (3.3), and (3.6) in the case of Problem II. The set of admissible elements is denoted by $\mathcal{V}_{a d}$.

DEFINITION 3.4. An element $\widehat{\mathbf{w}} \in \mathcal{V}_{a d}$ is called a solution of Problem I if

$$
\mathcal{J}_{N}(\widehat{\mathbf{w}})=\inf _{\mathbf{w} \in \mathcal{V}_{a d}} \mathcal{J}_{N}(\mathbf{w}),
$$

where $\mathcal{J}_{N}$ is defined by (3.4). An element $\widehat{\mathbf{w}} \in \mathcal{V}_{a d}$ is called a solution of Problem II if

$$
\mathcal{J}(\widehat{\mathbf{w}})=\inf _{\mathbf{w} \in \mathcal{V}_{a d}} \mathcal{J}(\mathbf{w}),
$$

where $\mathcal{J}$ is defined by (3.5).

4. An extension theorem, solutions of the Navier-Stokes equations, and the stress vector on the boundary. Our aim is to prove the existence of optimal solutions for Problems I and II and to obtain optimality systems of partial differential equations that optimal solutions must satisfy. To this end, we first prove three results that are of considerable interest in their own right in the study of Dirichlet boundary value problems for the Navier-Stokes equations.

The first result (section 4.1) is the identification of the trace space of $\mathcal{V}^{(1)}\left(Q_{T}\right)=$ $L^{2}\left(0, T ; \mathbf{V}^{1}(\Omega)\right) \cap H^{1}\left(0, T ; \mathbf{V}^{-1}(\Omega)\right)$, i.e., the collection of velocity boundary data that can be extended into functions belonging to $\mathcal{V}^{(1)}\left(Q_{T}\right)$. The second result (section 4.2) is the existence of a solution of the Navier-Stokes equations with boundary values in these trace spaces along with a priori estimates for the solution. The third result (section 4.3) is the identification of the space in which the trace of the stress vector (on the boundary) of admissible solutions is well defined.

4.1. An extension theorem for boundary data. We prove some results concerning the extension of functions from the lateral surface of the time-space cylinder to the entire cylinder, i.e., from $(0, T) \times \partial \Omega$ to $(0, T) \times \Omega$.

We set $Q=\mathbb{R} \times \Omega$ (the infinite time-space cylinder) and $S=\mathbb{R} \times \partial \Omega$ (the lateral surface of the infinite time-space cylinder). The problem we want to consider is to describe the space of vector fields defined on $S$ which can be extended to solenoidal vector fields defined on $Q$ which belong to the space $\mathcal{V}^{(1)}(Q)$, where we recall, from section 3 , the definition

$$
\mathcal{V}^{(s)}(Q)=\left\{\mathbf{v} \in L^{2}\left(\mathbb{R} ; \mathbf{V}^{(s)}(\Omega)\right): \partial_{t} \mathbf{v} \in L^{2}\left(\mathbb{R} ; \mathbf{V}^{(s-2)}(\Omega)\right)\right\} .
$$

Alternatively, the task here is to characterize the trace space of $\mathcal{V}^{(1)}(Q)$. We will see that it is necessary to examine the normal trace and tangential trace separately, as they belong to different function spaces.

We denote by $\boldsymbol{\tau}=\left(\tau_{1}, \tau_{2}\right)^{T}$ and $\mathbf{n}=\left(n_{1}, n_{2}\right)^{T}$ the unit counterclockwise tangent and outward normal vectors, respectively, along $\partial \Omega$. We have the following relations:

$$
\tau_{1}=n_{2} \quad \text { and } \quad \tau_{2}=-n_{1} .
$$

Given a boundary vector field

$$
\mathbf{b}(t, \mathbf{x})=b_{n}(t, \mathbf{x}) \mathbf{n}(\mathbf{x})+b_{\tau}(t, \mathbf{x}) \boldsymbol{\tau}(\mathbf{x}) \quad \text { a.e. }(t, \mathbf{x}) \in S
$$


satisfying

$$
\int_{\partial \Omega} \mathbf{b} \cdot \mathbf{n} d s=0 \quad \text { a.e. } t \in \mathbb{R}
$$

where $b_{n}=\mathbf{b} \cdot \mathbf{n}$ and $b_{\tau}=\mathbf{b} \cdot \boldsymbol{\tau}$, we seek a solenoidal extension $\mathbf{u}=\left(u_{1}, u_{2}\right)^{T} \in \mathcal{V}^{(1)}(Q)$ of the form (see [19])

$$
u_{1}=\partial_{2} F \quad \text { and } \quad u_{2}=-\partial_{1} F,
$$

where $F$ is the streamfunction for $\mathbf{u}$ and $\partial_{i} F=\partial F / \partial x_{i}$. In other words, given a boundary vector field $\mathbf{b}$ satisfying (4.3), we seek an $F$ such that

$$
b_{n}=-\left.(\nabla F \cdot \tau)\right|_{S} \equiv-\left.\partial_{\tau} F\right|_{S}
$$

and

$$
b_{\tau}=\left.\left.(\nabla F \cdot \mathbf{n})\right|_{S} \equiv \partial_{n} F\right|_{S} .
$$

Note that the assumption (4.3) is necessary since we are seeking a solenoidal extension of the boundary data $\mathbf{b}$.

With the assumption (4.3), the relation (4.5) is equivalent to

$$
\left.F\right|_{S}=h \equiv-\int_{\mathbf{x}_{0}}^{\mathbf{x}} b_{n}(t, \mathbf{x}(s)) d s,
$$

where the line integral is taken along $\partial \Omega$ in the counterclockwise direction starting from a fixed point $\mathbf{x}_{0} \in \partial \Omega$. Thus, for each given pair $\left(b_{\tau}, h\right)$ defined on $S$, we want to construct an $F \in \mathcal{H}^{(2)}(Q)$ satisfying (4.6) and (4.7), where

$$
\mathcal{H}^{(s)}(\mathbb{R} \times \Theta)=\left\{u \in L^{2}\left(\mathbb{R} ; H^{s}(\Theta)\right): \partial_{t} u \in L^{2}\left(\mathbb{R} ; H^{s-2}(\Theta)\right)\right\} .
$$

Here $s \in \mathbb{R}, \Theta$ is any spatial domain, and the norm on $\mathcal{H}^{(s)}(\mathbb{R} \times \Theta)$ is defined by

$$
\|F\|_{\mathcal{H}^{(s)}(\mathbb{R} \times \Theta)}^{2}=\|F\|_{L^{2}\left(\mathbb{R} ; H^{s}(\Theta)\right)}^{2}+\left\|\partial_{t} F\right\|_{L^{2}\left(\mathbb{R} ; H^{s-2}(\Theta)\right)}^{2} \quad \forall F \in \mathcal{H}^{(s)}(\mathbb{R} \times \Theta) .
$$

We now prove that such an extension $F$ exists provided that the boundary data $\left(b_{\tau}, h\right)$ belongs to an appropriate function space.

Proposition 4.1. A pair of functions $\left(b_{\tau}, h\right)$ defined on $S$ possess an extension $F \in \mathcal{H}^{(2)}(Q)$ satisfying (4.6), (4.7),

$$
\begin{aligned}
\|F\|_{\mathcal{H}^{(2)}(Q)}^{2} \leq C\{ & \left\|b_{\tau}\right\|_{L^{2}\left(\mathbb{R} ; H^{1 / 2}(\partial \Omega)\right)}^{2}+\left\|b_{\tau}\right\|_{H^{1 / 4}\left(\mathbb{R} ; L^{2}(\partial \Omega)\right)}^{2} \\
& \left.+\|h\|_{L^{2}\left(\mathbb{R} ; H^{3 / 2}(\partial \Omega)\right)}^{2}+\|h\|_{H^{3 / 4}\left(\mathbb{R} ; L^{2}(\partial \Omega)\right)}^{2}\right\},
\end{aligned}
$$

and

$$
F \text { vanishes outside a neighborhood of } S=\mathbb{R} \times \partial \Omega,
$$

where $C$ is a constant independent of $b_{\tau}, h$, and $F$, if and only if

$$
b_{\tau} \in L^{2}\left(\mathbb{R} ; H^{1 / 2}(\partial \Omega)\right) \cap H^{1 / 4}\left(\mathbb{R} ; L^{2}(\partial \Omega)\right)
$$


and

$$
h \in L^{2}\left(\mathbb{R} ; H^{3 / 2}(\partial \Omega)\right) \cap H^{3 / 4}\left(\mathbb{R} ; L^{2}(\partial \Omega)\right) .
$$

Proof. Given $b_{\tau}$ and $h$ satisfying (4.10)-(4.11), we construct an extension $F$ satisfying (4.6)-(4.9); the converse result is easily proved as well. $\partial \Omega$ being of class $C^{\infty}$, we may choose a neighborhood $U$ of $\partial \Omega$ and a coordinate system $\left(x_{1}^{\prime}, x_{2}^{\prime}\right)^{T}$ such that $U=\left\{\mathbf{x}=\left(x_{1}^{\prime}, x_{2}^{\prime}\right)^{T}:\left(x_{1}^{\prime}, 0\right)^{T} \in \partial \Omega, x_{2}^{\prime} \in[0, \epsilon]\right\}$ for some $\epsilon>0$. The space $\mathcal{H}^{(2)}(\mathbb{R} \times U)$ can be rewritten in the form

$$
\begin{aligned}
\mathcal{H}^{(2)}(\mathbb{R} \times U) \\
=\left\{F\left(x_{2}^{\prime}, t, x_{1}^{\prime}\right) \in L^{2}\left(0, \epsilon ; L^{2}\left(\mathbb{R} ; H^{2}(\partial \Omega)\right)\right) \cap L^{2}\left(0, \epsilon ; H^{1}\left(\mathbb{R} ; L^{2}(\partial \Omega)\right)\right):\right. \\
\left.\quad \partial_{x_{2}^{\prime} x_{2}^{\prime}} F \in L^{2}\left(0, \epsilon ; L^{2}\left(\mathbb{R} ; L^{2}(\partial \Omega)\right)\right)\right\} .
\end{aligned}
$$

By virtue of a trace theorem of [21], we have that the mappings $\gamma_{0}:\left.F \mapsto F\right|_{x_{2}^{\prime}=0}$ and $\gamma_{1}:\left.F \mapsto \partial_{x_{2}^{\prime}} F\right|_{x_{2}^{\prime}=0}$ are well defined on $\mathcal{H}^{(2)}(\mathbb{R} \times U)$; furthermore, the mapping

$$
\begin{aligned}
F \mapsto\left(\gamma_{0} F, \gamma_{1} F\right): & \\
\mathcal{H}^{(2)}(\mathbb{R} \times U) \rightarrow & {\left[L^{2}\left(\mathbb{R} ; H^{2}(\partial \Omega)\right) \cap H^{1}\left(\mathbb{R} ; L^{2}(\partial \Omega)\right), L^{2}\left(\mathbb{R} ; L^{2}(\partial \Omega)\right)\right]_{3 / 4} } \\
& \times\left[L^{2}\left(\mathbb{R} ; H^{2}(\partial \Omega)\right) \cap H^{1}\left(\mathbb{R} ; L^{2}(\partial \Omega)\right), L^{2}\left(\mathbb{R} ; L^{2}(\partial \Omega)\right)\right]_{1 / 4}
\end{aligned}
$$

is continuous and surjective. Here we have used the intermediate spaces $[\mathcal{X}, \mathcal{Y}]_{\alpha}$, $\alpha \in[0,1]$, of the Hilbert spaces $\mathcal{X}$ and $\mathcal{Y}$ as defined in [21]. Using the definition of these intermediate spaces (see [21]), we obtain

$$
\begin{aligned}
& {\left[L^{2}\left(\mathbb{R} ; H^{2}(\partial \Omega)\right) \cap H^{1}\left(\mathbb{R} ; L^{2}(\partial \Omega)\right), L^{2}\left(\mathbb{R} ; L^{2}(\partial \Omega)\right)\right]_{3 / 4}} \\
& \quad=L^{2}\left(\mathbb{R} ; H^{3 / 2}(\partial \Omega)\right) \cap H^{3 / 4}\left(\mathbb{R} ; L^{2}(\partial \Omega)\right)
\end{aligned}
$$

and

$$
\begin{aligned}
& {\left[L^{2}\left(\mathbb{R} ; H^{2}(\partial \Omega)\right) \cap H^{1}\left(\mathbb{R} ; L^{2}(\partial \Omega)\right), L^{2}\left(\mathbb{R} ; L^{2}(\partial \Omega)\right)\right]_{1 / 4}} \\
& \quad=L^{2}\left(\mathbb{R} ; H^{1 / 2}(\partial \Omega)\right) \cap H^{1 / 4}\left(\mathbb{R} ; L^{2}(\partial \Omega)\right) .
\end{aligned}
$$

Hence the mapping $F \mapsto\left(\gamma_{0} F, \gamma_{1} F\right)$ is continuous and surjective from $\mathcal{H}^{(2)}(\mathbb{R} \times U)$ to $\left[L^{2}\left(\mathbb{R} ; H^{3 / 2}(\partial \Omega)\right) \cap H^{3 / 4}\left(\mathbb{R} ; L^{2}(\partial \Omega)\right)\right] \times\left[L^{2}\left(\mathbb{R} ; \mathbf{H}^{1 / 2}(\partial \Omega)\right) \cap H^{1 / 4}\left(\mathbb{R} ; \mathbf{L}^{2}(\partial \Omega)\right)\right]$. Finally, we may choose another neighborhood $\widetilde{U}$ of $(0, \varepsilon) \times \partial \Omega$ such that the closure of $U$ is contained in $\widetilde{U}$. Well-known extension results allow us to extend continuously the space $\mathcal{H}^{(2)}(\mathbb{R} \times U)$ into the space $\left\{F \in \mathcal{H}^{(2)}(\mathbb{R} \times \Omega): F\right.$ vanishes outside $\left.\widetilde{U}\right\}$.

We are now in a position to prove the main extension result. We denote the finite time-space cylinder by $Q_{T}=(0, T) \times \Omega$ and its lateral surface by $S_{T}=(0, T) \times \partial \Omega$.

THEOREM 4.2. Assume that $b_{n}$ and $b_{\tau}$ satisfy

$$
\begin{gathered}
\int_{\partial \Omega} b_{n} d s=0 \quad \text { a.e. } t \in[0, T], \\
b_{n} \in L^{2}\left(0, T ; H^{1 / 2}(\partial \Omega)\right) \cap H^{3 / 4}\left(0, T ; H^{-1}(\partial \Omega)\right),
\end{gathered}
$$


and

$$
b_{\tau} \in L^{2}\left(0, T ; H^{1 / 2}(\partial \Omega)\right) \cap H^{1 / 4}\left(0, T ; L^{2}(\partial \Omega)\right) .
$$

Then, there exists a $\mathbf{u} \in \mathcal{V}^{(1)}\left(Q_{T}\right)$ satisfying

$$
\left.\mathbf{u}\right|_{S_{T}}=\mathbf{b} \equiv b_{n} \mathbf{n}+b_{\tau} \boldsymbol{\tau}
$$

and the estimate

$$
\begin{aligned}
\|\mathbf{u}\|_{\mathcal{V}^{(1)}\left(Q_{T}\right)}^{2} \leq C\{ & \left\|b_{n}\right\|_{L^{2}\left(0, T ; H^{1 / 2}(\partial \Omega)\right)}^{2}+\left\|b_{n}\right\|_{H^{3 / 4}\left(0, T ; H^{-1}(\partial \Omega)\right)}^{2} \\
& \left.+\left\|b_{\tau}\right\|_{L^{2}\left(0, T ; H^{1 / 2}(\partial \Omega)\right)}^{2}+\left\|b_{\tau}\right\|_{H^{1 / 4}\left(0, T ; L^{2}(\partial \Omega)\right)}^{2}\right\},
\end{aligned}
$$

where $C$ is a constant independent of $b_{n}$ and $b_{\tau}$, and such that $\mathbf{u}$ vanishes outside a neighborhood of $(0, T) \times \partial \Omega$.

Proof. By definition, the space $H^{r}\left(0, T ; H^{s}(\partial \Omega)\right)$ with fractional indices $r$ and $s$ is the restriction to $(0, T) \times \partial \Omega$ of $H^{r}\left(\mathbb{R} ; H^{s}(\partial \Omega)\right)$. Thus, we may extend the data in time; i.e., there exists a $\widetilde{b}_{n} \in L^{2}\left(\mathbb{R} ; H^{1 / 2}(\partial \Omega)\right) \cap H^{3 / 4}\left(\mathbb{R} ; H^{-1}(\partial \Omega)\right)$ and $\widetilde{b}_{\tau} \in L^{2}\left(\mathbb{R} ; H^{1 / 2}(\partial \Omega)\right) \cap H^{1 / 4}\left(\mathbb{R} ; L^{2}(\partial \Omega)\right)$ such that

$$
\begin{gathered}
\widetilde{b}_{n}=b_{n} \quad \text { and } \quad \widetilde{b}_{\tau}=b_{\tau} \quad \text { on }(0, T) \times \partial \Omega, \\
\left\|\widetilde{b}_{n}\right\|_{L^{2}\left(\mathbb{R} ; H^{1 / 2}(\partial \Omega)\right)}^{2}+\left\|\widetilde{b}_{n}\right\|_{H^{3 / 4}\left(\mathbb{R} ; H^{-1}(\partial \Omega)\right)}^{2} \\
\leq C\left\{\left\|b_{n}\right\|_{L^{2}\left(0, T ; H^{1 / 2}(\partial \Omega)\right)}^{2}+\left\|b_{n}\right\|_{H^{3 / 4}\left(0, T ; H^{-1}(\partial \Omega)\right)}^{2}\right\}
\end{gathered}
$$

and

$$
\begin{aligned}
& \left\|\widetilde{b}_{\tau}\right\|_{L^{2}\left(\mathbb{R} ; H^{1 / 2}(\partial \Omega)\right)}^{2}+\left\|\widetilde{b}_{\tau}\right\|_{H^{1 / 4}\left(\mathbb{R} ; L^{2}(\partial \Omega)\right)}^{2} \\
& \quad \leq C\left\{\left\|b_{\tau}\right\|_{L^{2}\left(0, T ; H^{1 / 2}(\partial \Omega)\right)}^{2}+\left\|b_{\tau}\right\|_{H^{1 / 4}\left(0, T ; L^{2}(\partial \Omega)\right)}^{2}\right\} .
\end{aligned}
$$

Furthermore, we may assume, without loss of generality, that

$$
\int_{\partial \Omega} \widetilde{b}_{n} d s=0 \quad \text { a.e. } t \in \mathbb{R} .
$$

Indeed, we can reset $\widetilde{\widetilde{b}}_{n}=\widetilde{b}_{n}-\left(\int_{\partial \Omega} \widetilde{b}_{n} d s / \int_{\partial \Omega} d s\right)$, if necessary. We define

$$
\widetilde{h}(t, \mathbf{x})=-\int_{\mathbf{x}_{0}}^{\mathbf{x}} \widetilde{b}_{n}(t, \mathbf{x}(s)) d s \quad \forall \mathbf{x} \in \partial \Omega,
$$

where the line integral on the right-hand side is taken counterclockwise along $\partial \Omega$, starting from a given point $\mathbf{x}_{0} \in \partial \Omega$. Evidently, $\widetilde{h} \in L^{2}\left(\mathbb{R} ; H^{3 / 2}(\partial \Omega)\right) \cap$ $H^{3 / 4}\left(\mathbb{R} ; L^{2}(\partial \Omega)\right)$. Then, Proposition 4.1 implies that there exists an $F \in \mathcal{H}^{(2)}(Q)$ which vanishes outside a neighborhood of $\mathbb{R} \times \partial \Omega$ such that

$$
\left.F\right|_{S}=\widetilde{h} \quad \text { and }\left.\quad \partial_{n} F\right|_{S}=\widetilde{b}_{\tau} .
$$

By setting

$$
\mathbf{u}=\operatorname{curl} F=\left(\begin{array}{c}
\partial_{2} F \\
-\partial_{1} F
\end{array}\right)
$$


we see that

$$
\begin{gathered}
\mathbf{u} \in \mathcal{V}^{(1)}(Q), \\
\left.(\mathbf{u} \cdot \mathbf{n})\right|_{S}=\left.\operatorname{curl} F \cdot \mathbf{n}\right|_{S}=-\nabla F \cdot \boldsymbol{\tau}=-\partial_{\tau} F=-\partial_{\tau} \widetilde{h}=\widetilde{b}_{n},
\end{gathered}
$$

and

$$
\left.(\mathbf{u} \cdot \boldsymbol{\tau})\right|_{S}=\left.\operatorname{curl} F \cdot \boldsymbol{\tau}\right|_{S}=\nabla F \cdot \mathbf{n}=\partial_{n} F=\widetilde{b}_{\tau} .
$$

Hence, $\left.\mathbf{u}\right|_{S_{T}}=\widetilde{\mathbf{b}} \equiv \widetilde{b}_{n} \mathbf{n}+\widetilde{b}_{\tau} \boldsymbol{\tau}$; i.e., $\mathbf{u}$ satisfies (4.15). The estimate (4.16) follows from Proposition 4.1.

REMARK. We see from the proofs of Proposition 4.1 and Theorem 4.2 that the restriction operator $\left.\mathbf{u} \mapsto(\mathbf{u} \cdot \mathbf{n})\right|_{\partial \Omega}$ is continuous from $\mathcal{V}^{(1)}\left(Q_{T}\right)$ to $H^{3 / 4}\left(0, T ; H^{-1}(\partial \Omega)\right) \cap$ $L^{2}\left(0, T ; H^{1 / 2}(\partial \Omega)\right)$. Also, the trace operator $\left.b_{n} \mapsto b_{n}\right|_{t=0}$ is continuous from the space $H^{3 / 4}\left(0, T ; H^{-1}(\partial \Omega)\right) \cap L^{2}\left(0, T ; H^{1 / 2}(\partial \Omega)\right)$ to $H^{-1 / 2}(\partial \Omega)$ (see [21]). Hence, the composition of these two operators, i.e., the operator $\left.\mathbf{u} \mapsto\left[\left.(\mathbf{u} \cdot \mathbf{n})\right|_{\partial \Omega}\right]\right|_{t=0}$, is continuous from $\mathcal{V}^{(1)}\left(Q_{T}\right)$ to $H^{-1 / 2}(\partial \Omega)$. On the other hand, the composition of the operators $\left.\mathbf{u} \mapsto \mathbf{u}\right|_{t=0}$ and $\left.\left.\mathbf{u}\right|_{t=0} \mapsto\left(\left.\mathbf{u}\right|_{t=0} \cdot \mathbf{n}\right)\right|_{\partial \Omega}$ is continuous from $\mathcal{V}^{(1)}\left(Q_{T}\right)$ to $H^{-1 / 2}(\partial \Omega)$ (see Lemma 3.1 and [28]). Hence, using the denseness of $\mathbf{C}^{\infty}\left(Q_{T}\right) \cap \mathcal{V}^{(1)}\left(Q_{T}\right)$ in $\mathcal{V}^{(1)}\left(Q_{T}\right)$ we obtain the following compatibility condition for the extension $\mathbf{u}$ of Theorem 4.2:

$$
\left.\left(\left.\mathbf{u}\right|_{t=0} \cdot \mathbf{n}\right)\right|_{\partial \Omega}=\left.\left(\left.(\mathbf{u} \cdot \mathbf{n})\right|_{\partial \Omega}\right)\right|_{t=0} \quad \forall \mathbf{u} \in \mathcal{V}^{(1)}\left(Q_{T}\right) .
$$

4.2. Estimates for the solutions of the Navier-Stokes equations with nonhomogeneous Dirichlet boundary data. We now consider the boundary value problem for the Navier-Stokes equation in the form introduced in Definition 3.2. The boundary data $\mathbf{b}$ is assumed to satisfy the compatibility condition (4.12). Our goal here is, with the help of the extension theorem of section 4.1, to establish the existence of a solution for (3.1)-(3.3) and derive estimates for the solutions in the space of critical smoothness in terms of the data $\mathbf{w}_{0}$ and $\mathbf{b}$.

Let $b_{n}$ and $b_{\tau}$ be the normal and tangential components of the boundary value b. We assume that $b_{n}$ and $b_{\tau}$ satisfy (4.12)-(4.14) and that

$$
\mathbf{w}_{0} \in \mathbf{V}^{0}(\Omega) .
$$

We also assume the compatibility condition

$$
\left.\left(\mathbf{w}_{0} \cdot \mathbf{n}\right)\right|_{\partial \Omega}=\left.b_{n}\right|_{t=0}
$$

(see the remark at the end of section 4.1). We express the solution $\mathbf{w}$ of (3.1)-(3.3) in the form

$$
\mathbf{w}=\mathbf{u}+\boldsymbol{\eta},
$$

where $\mathbf{u} \in \mathcal{V}^{(1)}\left(Q_{T}\right)$ is the vector field constructed in Theorem 4.2 satisfying (4.15) and (4.16). Note that the fact that $\mathbf{u} \in \mathcal{V}^{(1)}(Q)$ implies that $\left.\mathbf{u}\right|_{t=0} \in \mathbf{L}^{2}(\Omega)$; see Lemma 3.1. Substituting $\mathbf{w}=\mathbf{u}+\boldsymbol{\eta}$ into (3.1)-(3.3), we obtain for $\boldsymbol{\eta}$

$$
\begin{aligned}
& \left\langle\partial_{t} \boldsymbol{\eta}(t), \mathbf{z}\right\rangle+\mu \int_{\Omega} \nabla \boldsymbol{\eta}(t): \nabla \mathbf{z} d \mathbf{x}+\int_{\Omega}\left(\left(\boldsymbol{\eta}(t)+\mathbf{u}(t)+\mathbf{v}_{\infty}\right) \cdot \nabla\right) \boldsymbol{\eta}(t) \cdot \mathbf{z} d \mathbf{x} \\
& \quad+\int_{\Omega}(\boldsymbol{\eta}(t) \cdot \nabla) \mathbf{u}(t) \cdot \mathbf{z} d \mathbf{x}=\langle\mathbf{f}(t), \mathbf{z}\rangle \quad \forall \mathbf{z} \in \mathbf{V}_{0}^{1}(\Omega), \text { a.e. } t \in(0, T),
\end{aligned}
$$




$$
\left.\boldsymbol{\eta}\right|_{S_{T}}=\mathbf{0} \quad \text { in } L^{2}\left(0, T ; \mathbf{H}^{1 / 2}(\partial \Omega)\right)
$$

and

$$
\left.\boldsymbol{\eta}\right|_{t=0}=\boldsymbol{\eta}_{0} \equiv \mathbf{w}_{0}-\left.\mathbf{u}\right|_{t=0} \quad \text { in } \mathbf{V}_{0}^{0}(\Omega)
$$

where

$$
\langle\mathbf{f}(t), \mathbf{z}\rangle=-\mu \int_{\Omega} \nabla \mathbf{u}(t): \nabla \mathbf{z} d \mathbf{x}-\left\langle\partial_{t} \mathbf{u}(t), \mathbf{z}\right\rangle-\int_{\Omega}\left[\left(\mathbf{u}(t)+\mathbf{v}_{\infty}\right) \cdot \nabla\right] \mathbf{u}(t) \cdot \mathbf{z} d \mathbf{x} .
$$

Lemma 4.3. Assume that the hypotheses of Theorem 4.2 hold. Let $\mathbf{u}$ be the vector field constructed in Theorem 4.2. Assume also that the compatibility condition (4.18) holds. Then, there exists a unique solution $\boldsymbol{\eta} \in \mathcal{V}^{(1)}\left(Q_{T}\right)$ of system (4.19)-(4.21). Moreover, $\boldsymbol{\eta}$ satisfies the estimate

$$
\begin{aligned}
& \left\|\partial_{t} \boldsymbol{\eta}\right\|_{L^{2}\left(0, T ; \mathbf{V}^{-1}(\Omega)\right)}^{2}+\|\boldsymbol{\eta}\|_{L^{\infty}\left(0, T ; \mathbf{V}^{0}(\Omega)\right)}^{2}+\|\boldsymbol{\eta}\|_{L^{2}\left(0, T ; \mathbf{V}^{1}(\Omega)\right)}^{2} \\
& \quad \leq A\left(\|\mathbf{f}\|_{L^{2}\left(0, T ; \mathbf{V}^{-1}(\Omega)\right)},\|\mathbf{u}\|_{\mathcal{V}^{(1)}\left(Q_{T}\right)},\left\|\boldsymbol{\eta}_{0}\right\|_{\mathbf{V}^{\mathrm{o}}(\Omega)},\left|\mathbf{v}_{\infty}\right|\right)
\end{aligned}
$$

where $A(\cdot, \cdot, \cdot, \cdot)$ is a continuous positive function defined on $\mathbb{R} \times \mathbb{R} \times \mathbb{R} \times \mathbb{R}$ and $A\left(\lambda_{1}, \lambda_{2}, \lambda_{3},\left|\mathbf{v}_{\infty}\right|\right) \rightarrow 0$ as $\boldsymbol{\lambda}=\left(\lambda_{1}, \lambda_{2}, \lambda_{3}\right) \rightarrow(0,0,0)$.

Proof. The existence and uniqueness of the solution $\boldsymbol{\eta} \in \mathcal{V}^{(1)}\left(Q_{T}\right)$ for (4.19)(4.21) can be proved in exactly the same way as that for the two-dimensional NavierStokes equations with homogeneous boundary conditions in exterior domains; see, e.g., [19] or [28]. We only need to prove the estimate. (Note that $\boldsymbol{\eta}_{0} \in \mathbf{V}_{0}^{0}(\Omega)$.)

Letting $\mathbf{z}=\boldsymbol{\eta}(t, \cdot)$ in (4.19) yields

$$
\begin{gathered}
\frac{1}{2} \partial_{t}\|\boldsymbol{\eta}(t, \cdot)\|_{\mathbf{L}^{2}(\Omega)}^{2}+\mu\|\nabla \boldsymbol{\eta}(t, \cdot)\|_{\mathbf{L}^{2}(\Omega)}^{2}=\langle\mathbf{f}(t, \cdot), \boldsymbol{\eta}(t, \cdot)\rangle-\int_{\Omega}(\boldsymbol{\eta} \cdot \nabla) \mathbf{u} \cdot \boldsymbol{\eta} d \mathbf{x} \\
\leq \frac{1}{\mu}\|\mathbf{f}(t, \cdot)\|_{\mathbf{V}^{-1}(\Omega)}^{2}+\frac{\mu}{4}\left(\|\nabla \boldsymbol{\eta}(t, \cdot)\|_{\mathbf{L}^{2}(\Omega)}^{2}+\|\boldsymbol{\eta}(t, \cdot)\|_{\mathbf{L}^{2}(\Omega)}^{2}\right) \\
+\|\nabla \mathbf{u}(t, \cdot)\|_{\mathbf{L}^{2}(\Omega)}\|\boldsymbol{\eta}(t, \cdot)\|_{\mathbf{L}^{4}(\Omega)}^{2} .
\end{gathered}
$$

Applying to the last term the Ladyzhenskaya inequality (see [19, Lemma 1])

$$
\|\eta\|_{L^{4}(\Omega)}^{2} \leq \sqrt{2}\|\eta\|_{L^{2}(\Omega)}\|\nabla \eta\|_{L^{2}(\Omega)} \quad \forall \eta \in H^{1}(\Omega)
$$

and then integrating with respect to $t$, we obtain

$$
\begin{aligned}
& \|\boldsymbol{\eta}(t, \cdot)\|_{\mathbf{L}^{2}(\Omega)}^{2}+\mu \int_{0}^{t}\|\nabla \boldsymbol{\eta}(t, \cdot)\|_{\mathbf{L}^{2}(\Omega)}^{2} d t \leq\left\|\boldsymbol{\eta}_{0}\right\|_{\mathbf{L}^{2}(\Omega)}^{2} \\
& \quad+\frac{2}{\mu} \int_{0}^{t}\|\mathbf{f}(\tau, \cdot)\|_{\mathbf{V}^{-1}(\Omega)}^{2} d \tau+\int_{0}^{t}\left(\frac{\mu}{2}+\frac{4}{\mu}\|\nabla \mathbf{u}(\tau, \cdot)\|_{\mathbf{L}^{2}(\Omega)}^{2}\right)\|\boldsymbol{\eta}(\tau, \cdot)\|_{\mathbf{L}^{2}(\Omega)}^{2} d \tau .
\end{aligned}
$$

Then, the Gronwall inequality yields the estimate

$$
\begin{aligned}
& \|\boldsymbol{\eta}\|_{L^{\infty}\left(0, T ; \mathbf{L}^{2}(\Omega)\right)}^{2}+\|\boldsymbol{\eta}\|_{L^{2}\left(0, T ; \mathbf{H}^{1}(\Omega)\right)}^{2} \\
& \quad \leq A_{1}\left(\|\mathbf{f}\|_{L^{2}\left(0, T ; \mathbf{V}^{-1}(\Omega)\right)},\|\mathbf{u}\|_{\mathbf{L}^{2}\left(0, T ; \mathbf{H}^{1}(\Omega)\right)},\left\|\boldsymbol{\eta}_{0}\right\|_{\mathbf{L}^{2}(\Omega)}\right)
\end{aligned}
$$

where $A_{1}(\cdot, \cdot, \cdot)$ is a continuous positive function defined on $\mathbb{R} \times \mathbb{R} \times \mathbb{R}$. Evidently, this last estimate implies

$$
\begin{aligned}
& \|\boldsymbol{\eta}\|_{L^{\infty}\left(0, T ; \mathbf{V}^{0}(\Omega)\right)}^{2}+\|\boldsymbol{\eta}\|_{L^{2}\left(0, T ; \mathbf{V}^{1}(\Omega)\right)}^{2} \\
& \quad \leq A_{1}\left(\|\mathbf{f}\|_{L^{2}\left(0, T ; \mathbf{V}^{-1}(\Omega)\right)},\|\mathbf{u}\|_{\mathcal{V}^{(1)}\left(Q_{T}\right)},\left\|\boldsymbol{\eta}_{0}\right\|_{\mathbf{V}^{0}(\Omega)}\right) .
\end{aligned}
$$


Now, taking the supremum of (4.19) with respect to $\mathbf{z} \in \mathbf{V}_{0}^{1}(\Omega)$ with $\|\mathbf{z}\|_{\mathbf{V}_{0}^{1}(\Omega)}=1$ and again applying the Ladyzhenskaya inequality we obtain

$$
\begin{aligned}
\left\|\partial_{t} \boldsymbol{\eta}(t, \cdot)\right\|_{\mathbf{V}^{-1}(\Omega)} \leq \mu & \|\boldsymbol{\eta}(t, \cdot)\|_{\mathbf{H}^{1}(\Omega)}+C\|\boldsymbol{\eta}(t, \cdot)\|_{\mathbf{H}^{1}(\Omega)}\|\boldsymbol{\eta}(t, \cdot)\|_{\mathbf{L}^{2}(\Omega)} \\
& +C\|\mathbf{u}(t, \cdot)\|_{\mathbf{H}^{1}(\Omega)}^{1 / 2}\|\mathbf{u}(t, \cdot)\|_{\mathbf{L}^{2}(\Omega)}^{1 / 2}\|\boldsymbol{\eta}(t, \cdot)\|_{\mathbf{H}^{1}(\Omega)}^{1 / 2}\|\boldsymbol{\eta}(t, \cdot)\|_{\mathbf{L}^{2}(\Omega)}^{1 / 2} \\
& +C\left|\mathbf{v}_{\infty}\right|\|\boldsymbol{\eta}(t, \cdot)\|_{\mathbf{L}^{2}(\Omega)}+\|\mathbf{f}(t, \cdot)\|_{\mathbf{V}^{-1}(\Omega)}
\end{aligned}
$$

so that

$$
\begin{aligned}
& \left\|\partial_{t} \boldsymbol{\eta}(t, \cdot)\right\|_{L^{2}\left(0, T ; \mathbf{V}^{-1}(\Omega)\right)}^{2} \\
& \leq C\|\boldsymbol{\eta}(t, \cdot)\|_{L^{2}\left(0, T ; \mathbf{H}^{1}(\Omega)\right)}^{2}+C\|\boldsymbol{\eta}(t, \cdot)\|_{L^{\infty}\left(0, T ; \mathbf{L}^{2}(\Omega)\right)}^{2}\|\boldsymbol{\eta}(t, \cdot)\|_{L^{2}\left(0, T ; \mathbf{H}^{1}(\Omega)\right)}^{2} \\
& \quad+C\|\mathbf{u}(t, \cdot)\|_{L^{\infty}\left(0, T ; \mathbf{L}^{2}(\Omega)\right)}\|\boldsymbol{\eta}(t, \cdot)\|_{L^{\infty}\left(0, T ; \mathbf{L}^{2}(\Omega)\right)} \\
& \quad \cdot\|\mathbf{u}(t, \cdot)\|_{L^{2}\left(0, T ; \mathbf{H}^{1}(\Omega)\right)}\|\boldsymbol{\eta}(t, \cdot)\|_{L^{2}\left(0, T ; \mathbf{H}^{1}(\Omega)\right)} \\
& \quad+C\left|\mathbf{v}_{\infty}\right|^{2}\|\boldsymbol{\eta}(t, \cdot)\|_{L^{2}\left(0, T ; \mathbf{L}^{2}(\Omega)\right)}^{2}+C\|\mathbf{f}(t, \cdot)\|_{L^{2}\left(0, T ; \mathbf{V}^{-1}(\Omega)\right)}^{2}
\end{aligned}
$$

Hence, using (4.23) and Lemma 3.1, we obtain the desired estimate.

Lemma 4.3 and Theorem 4.2 lead to the following result.

THEOREM 4.4. Let $\mathbf{b}$ and $\mathbf{w}_{0}$ satisfy (4.12)-(4.14) and (4.17)-(4.18). Then, there exists a unique solution $\mathbf{w} \in \mathcal{V}^{(1)}\left(Q_{T}\right)$ for the problem (3.1)-(3.3). Moreover, the solution satisfies the estimate

$$
\begin{gathered}
\|\mathbf{w}\|_{\mathcal{V}^{(1)}\left(Q_{T}\right)}^{2} \\
\leq B\left(\left\|\mathbf{w}_{0}\right\|_{\mathbf{L}^{2}(\Omega)},\left\|b_{n}\right\|_{L^{2}\left(0, T ; H^{1 / 2}(\partial \Omega)\right)}+\left\|b_{n}\right\|_{H^{3 / 4}\left(0, T ; H^{-1}(\partial \Omega)\right)},\right. \\
\left.\quad\left\|b_{\tau}\right\|_{L^{2}\left(0, T ; H^{1 / 2}(\partial \Omega)\right)}+\left\|b_{\tau}\right\|_{H^{1 / 4}\left(0, T ; L^{2}(\partial \Omega)\right)},\left|\mathbf{v}_{\infty}\right|\right)
\end{gathered}
$$

where $B(\cdot, \cdot, \cdot, \cdot)$ is a continuous positive function defined on $\mathbb{R} \times \mathbb{R} \times \mathbb{R} \times \mathbb{R}$.

Proof. Let $\mathbf{u} \in \mathcal{V}^{(1)}\left(Q_{T}\right)$ be the extension of the data $\mathbf{b}$ into $Q_{T}$ constructed in Theorem 4.2 and let $\boldsymbol{\eta}$ be the solution of (4.19)-(4.21) with $\mathbf{f}$ defined by (4.22). The existence and uniqueness of such an $\boldsymbol{\eta}$ is guaranteed by Lemma 4.3. Set $\mathbf{w}=\mathbf{u}+\boldsymbol{\eta}$; then $\mathbf{w}$ is clearly the unique solution of (3.1)-(3.3). Thus, it only remains to prove the estimate (4.24).

From (4.22) and the fact (see Theorem 4.2) that $\mathbf{u}$ has bounded support, we have that

$$
\begin{aligned}
&\|\mathbf{f}(t, \cdot)\|_{\mathbf{V}^{-1}(\Omega)} \leq \mu\|\mathbf{u}(t, \cdot)\|_{\mathbf{H}^{1}(\Omega)}+\left\|\partial_{t} \mathbf{u}(t, \cdot)\right\|_{\mathbf{V}^{-1}(\Omega)} \\
&+\|\mathbf{u}(t, \cdot)\|_{\mathbf{L}^{2}(\Omega)}\|\mathbf{u}(t, \cdot)\|_{\mathbf{H}^{1}(\Omega)}+\left|\mathbf{v}_{\infty}\right|\|\mathbf{u}(t, \cdot)\|_{\mathbf{H}^{1}(\Omega)} .
\end{aligned}
$$

Also, from (4.21), we have that

$$
\left\|\boldsymbol{\eta}_{0}\right\|_{\mathbf{L}^{2}(\Omega)} \leq\left\|\mathbf{w}_{0}\right\|_{\mathbf{L}^{2}(\Omega)}+\|\mathbf{u}(0, \cdot)\|_{\mathbf{L}^{2}(\Omega)} .
$$

Hence, (4.24) follows from Theorem 4.2, Lemmas 3.1 and 4.3, (4.25), and (4.26).

REMARK. We stress that the normal and tangential components of the boundary condition for the Navier-Stokes equations have different smoothness. This is a feature that is not exhibited in boundary value problems for general second-order parabolic systems. 
4.3. The stress vector (on the boundary) of admissible solutions. We now show that the stress vector on the boundary $\left.\left(-p I+\mu\left(\nabla \mathbf{w}+(\nabla \mathbf{w})^{T}\right)\right) \cdot \mathbf{n}\right|_{(0, T) \times \partial \Omega}$, where $\mathbf{w} \in Y$ is an admissible solution in the sense of Definition 3.3 and $p$ is an associated pressure field, is well defined in a certain function space. This result will be needed in section 6.4 in order to derive the optimality system in the form of a boundary value problem for a system of partial differential equations. Note that the requirement $\mathbf{w} \in \mathcal{V}_{a d}$ is stronger than $\mathbf{w} \in \mathcal{V}^{(1)}\left(Q_{T}\right)$ merely being a solution of (3.1) and (3.3). (We will actually show that each of $\left.(p \mathbf{n})\right|_{(0, T) \times \partial \Omega},\left.(\nabla \mathbf{w} \cdot \mathbf{n})\right|_{(0, T) \times \partial \Omega}$, and $\left.\left((\nabla \mathbf{w})^{T} \cdot \mathbf{n}\right)\right|_{(0, T) \times \partial \Omega}$ is well defined. $)$

Let $\mathbf{w} \in Y$ be an admissible element; then $\mathbf{w}$ satisfies (3.1) and (3.3). From the definition of $Y$ we see that $\left.\mathbf{w}\right|_{(0, T) \times \partial \Omega}$ is well defined and

$$
\left.\mathbf{w}\right|_{(0, T) \times \partial \Omega} \in H^{1}\left(0, T ; \mathbf{L}^{2}(\partial \Omega)\right) \cap L^{2}\left(0, T ; \mathbf{H}^{1 / 2}(\partial \Omega)\right) \cap \mathbf{L}^{k}((0, T) \times \partial \Omega) .
$$

By de Rham's lemma (see [14] and [28]), there exists a $p \in L^{2}\left(0, T ; L_{\text {loc }}^{2}(\Omega)\right)$ such that $\nabla p \in L^{2}\left(0, T ; \mathbf{H}^{-1}(\Omega)\right)$ and

$$
\partial_{t} \mathbf{w}-\mu \Delta \mathbf{w}+\left[\left(\mathbf{w}+\mathbf{v}_{\infty}\right) \cdot \nabla\right] \mathbf{w}+\nabla p=\mathbf{0}
$$

in the sense of distributions on $Q_{T}$. To study the normal stress on $\partial \Omega$, the behavior of $\mathbf{w}$ and $p$ at infinity is irrelevant and we can restrict our attention to a bounded domain whose boundary contains $\partial \Omega$. To this end, we let $\Theta \subset \Omega$ be a bounded domain with $C^{\infty}$ boundary $\partial \Theta$ such that $\partial \Theta \cap \partial \Omega=\partial \Omega$. We denote by $\gamma$ the restriction operator on $\partial \Theta$. Let $F$ be a streamfunction of $\mathbf{w}$ which can constructed as in section 4.1, satisfying on $\Theta$

$$
w_{1}=\partial_{2} F \quad \text { and } \quad w_{2}=-\partial_{1} F .
$$

Since $\mathbf{w} \in Y\left(Q_{\Theta}\right) \subset \mathcal{V}^{(1)}\left(Q_{\Theta}\right)$, where $Q_{\Theta}=(0, T) \times \Theta$, we have $F \in \mathcal{H}^{(2)}\left(Q_{\Theta}\right)$. The restriction of (4.27) and the divergence-free condition for $\mathbf{w}$ on $Q_{\Theta}$ yields

$$
\partial_{t} \mathbf{w}-\mu \Delta \mathbf{w}+\left[\left(\mathbf{w}+\mathbf{v}_{\infty}\right) \cdot \nabla\right] \mathbf{w}+\nabla p=\mathbf{0}
$$

and

$$
\operatorname{div} \mathbf{w}=0,
$$

where the derivatives are understood in the sense of distributions in $Q_{\Theta}$. Applying the curl operator to (4.29) and taking into account (4.28) we obtain

$$
\partial_{t} \Delta F-\mu \Delta^{2} F=G,
$$

where

$$
G=-\operatorname{curl}\left\{\left[\left(\mathbf{w}+\mathbf{v}_{\infty}\right) \cdot \nabla\right] \mathbf{w}\right\}=-\left(w_{1}+v_{\infty, 1}\right) \Delta w_{2}+\left(w_{2}+v_{\infty, 2}\right) \Delta w_{1} .
$$

Lemma 4.5. Assume $\mathbf{w} \in Y$ is a solution of (3.1) and $G$ is defined by (4.32). Then, $G \in L^{1}\left(0, T ; W^{-1, \alpha}(\Theta)\right)$ for every $\alpha \in(1,2)$.

Proof. Let $\alpha^{\prime}$ and $\beta$ be defined by

$$
\frac{1}{\alpha^{\prime}}+\frac{1}{\alpha}=1 \quad \text { and } \quad \frac{1}{\beta}+\frac{1}{\alpha^{\prime}}=\frac{1}{2} .
$$


Let $\phi \in W_{0}^{1, \alpha^{\prime}}(\Theta)$ be given. By integration by parts and Hölder's inequality, we have that a.e. $t \in(0, T)$,

$$
\begin{aligned}
&\left|\int_{\Theta}\left(w_{1}+v_{\infty, 1}\right) \Delta w_{2} \phi d \mathbf{x}\right| \\
&=\left|\int_{\Theta}\left(\phi \nabla w_{1} \cdot \nabla w_{2}+\left(w_{1}+v_{\infty, 1}\right) \nabla w_{2} \cdot \nabla \phi\right) d \mathbf{x}\right| \\
& \quad \leq\left\|\nabla w_{1}\right\|_{\mathbf{L}^{2}(\Theta)}\left\|\nabla w_{2}\right\|_{\mathbf{L}^{2}(\Theta)}\|\phi\|_{L^{\infty}(\Theta)} \\
& \quad+\left\|w_{1}+v_{\infty, 1}\right\|_{L^{\beta}(\Theta)}\left\|\nabla w_{2}\right\|_{\mathbf{L}^{2}(\Theta)}\|\nabla \phi\|_{\mathbf{L}^{\alpha^{\prime}}(\Theta)}
\end{aligned}
$$

Since $\alpha^{\prime} \in(2, \infty)$ and $\beta \in(2, \infty)$, Sobolev imbedding theorems imply

$$
\|\phi\|_{L^{\infty}(\Theta)} \leq C\|\phi\|_{W^{1, \alpha^{\prime}}(\Theta)} \quad \text { and } \quad\left\|w_{1}+v_{\infty, 1}\right\|_{L^{\beta}(\Theta)} \leq C\left\|w_{1}+v_{\infty, 1}\right\|_{H^{1}(\Theta)}
$$

so that, from (4.33),

$$
\left\|\left(w_{1}+v_{\infty, 1}\right) \Delta w_{2}\right\|_{L^{1}\left(0, T ; W^{-1, \alpha}(\Theta)\right)} \leq C\left(\|\mathbf{w}\|_{\mathcal{V}^{(1)}\left(Q_{\Theta}\right)}^{2}+\left|\mathbf{v}_{\infty}\right|^{2}\right) .
$$

Similarly, we can show

$$
\left\|\left(w_{2}+v_{\infty, 2}\right) \Delta w_{1}\right\|_{L^{1}\left(0, T ; W^{-1, \alpha}(\Theta)\right)} \leq C\left(\|\mathbf{w}\|_{\mathcal{V}^{(1)}\left(Q_{\Theta}\right)}^{2}+\left|\mathbf{v}_{\infty}\right|^{2}\right) .
$$

It follows from the last two inequalities and (4.32) that $G \in L^{1}\left(0, T ; W^{-1, \alpha}(\Theta)\right)$. 口

Since $F \in \mathcal{H}^{(2)}\left(Q_{\Theta}\right)$, we have $\Delta F \in L^{2}\left(0, T ; L^{2}(\Theta)\right)$. From (4.31), (4.32), and Lemma 4.5 , we see that

$$
\Delta\left(\partial_{t} F-\mu \Delta F\right) \in L^{1}\left(0, T ; W^{-1, \alpha}(\Theta)\right) .
$$

We now introduce the space

$$
X_{\alpha}=\left\{f \in L^{2}(\Theta): \Delta f \in W^{-1, \alpha}(\Theta)\right\}
$$

equipped with the norm

$$
\|f\|_{X_{\alpha}}=\|f\|_{L^{2}(\Theta)}+\|\Delta f\|_{W^{-1, \alpha}(\Theta)} \quad \forall f \in X_{\alpha} .
$$

It is easy to verify that $X_{\alpha}$ is a Banach space. We will establish a trace theorem for $X_{\alpha}$. To this end, we first prove two lemmas.

LEMMA 4.6. Every bounded linear functional $L$ on $X_{\alpha}$ has the representation

$$
L f=(f, \phi)+\langle\Delta f, \psi\rangle \quad \forall f \in X_{\alpha},
$$

where $\phi \in L^{2}(\Theta), \psi \in W_{0}^{1, \alpha^{\prime}}(\Theta),(\cdot, \cdot)$ denotes the $L^{2}(\Theta)$-inner product, and $\langle\cdot, \cdot \cdot\rangle$ denotes the duality pairing between $W^{-1, \alpha}(\Theta)$ and $W_{0}^{1, \alpha^{\prime}}(\Theta)$.

Proof. In $L^{2}(\Theta) \times W^{-1, \alpha}(\Theta)$, we consider the subspace $\Pi=\{(f, \Delta f): f \in$ $\left.X_{\alpha}\right\}$. Clearly, $\Pi$ is closed under the Cartesian norm for $L^{2}(\Theta) \times W^{-1, \alpha}(\Theta)$ and the mapping $\pi: f \mapsto(f, \Delta f)$ establishes an isomorphism between $X_{\alpha}$ and $\Pi$. Let an arbitrary bounded linear functional $L$ on $X_{\alpha}$ be given. Then, there exists a unique functional $K$ on $\Pi$ such that $L f=K(f, \Delta f)$. Using the Hahn-Banach theorem we can extend the functional $K$ defined on $\Pi$ into a functional $\widetilde{K}$ defined on the entire space $L^{2}(\Theta) \times W^{-1, \alpha}(\Theta)$ with the functional norm preserved, i.e., with $\|\widetilde{K}\|=\|K\|$. 
Since $L^{2}(\Theta) \times W^{-1, \alpha}(\Theta)$ is reflexive, there exist $\phi \in L^{2}(\Theta)$ and $\psi \in W_{0}^{1, \alpha^{\prime}}(\Theta)$ such that

$$
\widetilde{K}(f, g)=(f, \phi)+\langle g, \psi\rangle \quad \forall(f, g) \in L^{2}(\Theta) \times W^{-1, \alpha}(\Theta),
$$

so that on the subspace $\Pi$,

$$
K(f, \Delta f)=(f, \phi)+\langle\Delta f, \psi\rangle \quad \forall(f, \Delta f) \in \Pi .
$$

The last relation is equivalent to (4.34).

LEMma 4.7. $C^{\infty}(\bar{\Theta})$ is dense in $X_{\alpha}$.

Proof. We need only show that if a bounded linear functional $L$ on $X_{\alpha}$ satisfies $L f=0$ for all $f \in C^{\infty}(\bar{\Theta})$, then $L=0$. We assume that $L$ is a bounded linear functional on $X_{\alpha}$ satisfying $L f=0$ for all $f \in C^{\infty}(\bar{\Theta})$. By Lemma 4.6, there exist $\phi \in L^{2}(\Theta)$ and $\psi \in W_{0}^{1, \alpha^{\prime}}(\Theta)$ such that

$$
L f=(f, \phi)+\langle\Delta f, \psi\rangle=0 \quad \forall f \in C^{\infty}(\bar{\Theta}) .
$$

This implies that, in the sense of distributions,

$$
\Delta \psi=-\phi .
$$

As $\psi \in W_{0}^{1, \alpha^{\prime}}(\Theta)$ and $\phi \in L^{2}(\Theta)$, we deduce from elliptic regularity that $\psi \in$ $W_{0}^{1, \alpha^{\prime}}(\Theta) \cap H^{2}(\Theta)$ and $\Delta \psi=-\phi$ in $L^{2}(\Theta)$, which in turn implies $\partial_{n} \psi \in H^{1 / 2}(\partial \Omega)$. For each $f \in C^{\infty}(\bar{\Theta})$, we are justified in using integration by parts to obtain

$$
\begin{aligned}
0=(f, \phi)+\langle\Delta f, \psi\rangle & =(f, \phi)+(\Delta f, \psi) \\
& =(f, \phi)+(f, \Delta \psi)-\left\langle\partial_{n} \psi, f\right\rangle \\
& =(f, \phi)+(f,-\phi)-\left\langle\partial_{n} \psi, f\right\rangle=-\left\langle\partial_{n} \psi, f\right\rangle
\end{aligned}
$$

so that $\left.\partial_{n} \psi\right|_{\partial \Omega}=0$ and $\psi \in H_{0}^{2}(\Theta)$. Using the denseness of $C_{0}^{\infty}(\Theta)$ in $H_{0}^{2}(\Theta)$ we may choose a sequence $\left\{\psi_{n}\right\} \subset C_{0}^{\infty}(\Theta)$ such that $\psi_{n} \rightarrow \psi$ in $H^{2}(\bar{\Theta})$. Then, for each $f \in X_{\alpha}$ we have

$$
\begin{aligned}
(f, \phi)+\langle\Delta f, \psi\rangle & =(f, \phi)+\lim _{n \rightarrow \infty}\left\langle\Delta f, \psi_{n}\right\rangle \\
& =(f, \phi)+\lim _{n \rightarrow \infty}\left(f, \Delta \psi_{n}\right)=(f, \phi)+(f, \Delta \psi)=(f, \phi)+(f,-\phi)=0 ;
\end{aligned}
$$

i.e., we have shown that

$$
L f=0 \quad \forall f \in X_{\alpha} .
$$

Hence, $L=0$.

In the sequel, we will make use of Besov spaces $B^{s, q}(\partial \Theta)$, where $s$ is the smoothness index and $q$ is the integrability index. For the definition of Besov spaces, see [4] and [29], where the Besov spaces $B^{s, q}(\partial \Theta)$ are denoted by $B_{q, q}^{s}(\partial \Theta)$. One can also consult [1] for the definition of Besov spaces and the relations between Besov spaces and Sobolev spaces. One important feature of Besov spaces is that they coincide with the traces of Sobolev spaces. In particular, we have the following precise result: if we denote by $\gamma$ the mapping $\gamma f=\left.f\right|_{\partial \Theta}$ for functions defined in $\Theta$, then the mapping

$$
\left(\gamma, \gamma \partial_{n}\right): W^{2, \alpha^{\prime}}(\Theta) \rightarrow B^{2-1 / \alpha^{\prime}, \alpha^{\prime}}(\partial \Theta) \times B^{1-1 / \alpha^{\prime}, \alpha^{\prime}}(\partial \Theta)
$$

is continuous and establishes an epimorphism; see [4] and [29]. 
Proposition 4.8. Assume that $1<\alpha<2$. Then, the operator $\gamma$, defined on $C^{\infty}(\bar{\Theta})$ by $\gamma f=\left.f\right|_{\partial \Theta}$, can be extended continuously into the trace operator

$$
\gamma \in \mathcal{L}\left(X_{\alpha} ; B^{-1 / \alpha, \alpha}(\partial \Theta)\right)
$$

Proof. By (4.35), we can choose a continuous linear operator

$$
K: B^{1-1 / \alpha^{\prime}, \alpha^{\prime}}(\partial \Theta) \rightarrow W^{2, \alpha^{\prime}}(\Theta)
$$

such that

$$
\gamma K \phi=0 \quad \text { and } \quad \gamma \partial_{n} K \phi=\phi \quad \forall \phi \in B^{1-1 / \alpha^{\prime}, \alpha^{\prime}}(\partial \Theta) .
$$

Let $f \in X_{\alpha}$. We define a linear functional $Z$ on $B^{1-1 / \alpha^{\prime}, \alpha^{\prime}}(\partial \Theta)$ by

$$
Z \phi=Z_{K}(\phi)=(f, \Delta K \phi)-\langle\Delta f, K \phi\rangle \quad \forall \phi \in B^{1-1 / \alpha^{\prime}, \alpha^{\prime}}(\partial \Theta) .
$$

We claim that $Z$ does not depend on the choice of $K$. Indeed, let $K_{1}$ and $K_{2}$ be two continuous linear operators satisfying (4.37)-(4.38). Then, by (4.38),

$$
\gamma\left(K_{1}-K_{2}\right) \phi=0 \quad \text { and } \quad \gamma \partial_{n}\left(K_{1}-K_{2}\right) \phi=0 \quad \forall \phi \in B^{1-1 / \alpha^{\prime}, \alpha^{\prime}}(\partial \Theta)
$$

so that if $f \in C^{\infty}(\bar{\Theta})$, then integration by parts yields

$$
Z_{K_{1}}(\phi)-Z_{K_{2}}(\phi)=\left(f, \Delta\left(K_{1}-K_{2}\right) \phi\right)-\left\langle\Delta f,\left(K_{1}-K_{2}\right) \phi\right\rangle=0 .
$$

By virtue of Lemma 4.7, this equality is true for an arbitrary $f \in X_{\alpha}$. Hence, we have shown that $Z_{K_{1}}=Z_{K_{2}}$, i.e., that the operator $Z$ is well defined. Evidently, $Z_{K}$ is bounded on $B^{1-1 / \alpha^{\prime}, \alpha^{\prime}}(\partial \Theta)$. Hence, by the Riesz theorem, there exists an element $R f \in B^{-1 / \alpha, \alpha}(\partial \Theta)$ such that

$$
(R f, \phi)=Z(\phi)=(f, \Delta K \phi)-\langle\Delta f, K \phi\rangle \quad \forall \phi \in B^{1-1 / \alpha^{\prime}, \alpha^{\prime}}(\partial \Theta),
$$

where $R$ is the Riesz map. If $f \in C^{\infty}(\bar{\Theta})$, then using Green's formula in the last equation we obtain $R f=\gamma f$. By virtue of Lemma 4.7 and the boundedness of the operator in (4.37), we can extend the operator $\gamma$ continuously into the mapping of (4.36).

We introduce the set

$$
\Upsilon=\{\mathbf{w} \in Y: \mathbf{w} \text { satisfies }(3.1)\}
$$

equipped with the topology generated by the norm of $Y$.

THEOREM 4.9. Let $\mathbf{w} \in Y$ be a solution of (3.1) and $F \in \mathcal{H}^{(2)}\left(Q_{\Theta}\right)$ be defined by (4.28). Let $G \in L^{1}\left(0, T ; W^{-1, \alpha}(\Theta)\right), \alpha \in(1,2)$, be defined by (4.32). Then, $\gamma(\nabla \mathbf{w} \cdot \mathbf{n}) \in L^{1}\left(0, T ; \mathbf{B}^{-1 / \alpha, \alpha}(\partial \Theta)\right)$ and $\gamma\left((\nabla \mathbf{w})^{T} \cdot \mathbf{n}\right) \in L^{1}\left(0, T ; \mathbf{B}^{-1 / \alpha, \alpha}(\partial \Theta)\right)$. Moreover, the mappings $\mathbf{w} \mapsto \gamma(\nabla \mathbf{w} \cdot \mathbf{n})$ and $\mathbf{w} \mapsto \gamma\left((\nabla \mathbf{w})^{T} \cdot \mathbf{n}\right)$ are continuous from the topological space $\Upsilon$ to $L^{1}\left(0, T ; \mathbf{B}^{-1 / \alpha, \alpha}(\partial \Theta)\right)$.

Proof. From the assumptions on $F$ and $G$, we easily deduce that $\partial_{t} F-\mu \Delta F \in$ $L^{2}\left(0, T ; L^{2}(\Theta)\right)$ and $\Delta\left(\partial_{t} F-\mu \Delta F\right) \in L^{1}\left(0, T ; W^{-1, \alpha}(\Theta)\right)$. Hence, Proposition 4.8 implies that for almost every $t \in(0, T)$, the restriction $\gamma\left(\partial_{t} F(t, \cdot)-\mu \Delta F(t, \cdot)\right)$ is well defined and

$$
\gamma\left(\partial_{t} F-\mu \Delta F\right) \in L^{1}\left(0, T ; B^{-1 / \alpha, \alpha}(\partial \Theta)\right),
$$


where $1<\alpha<2$. Since $F \in \mathcal{H}^{(2)}\left(Q_{\Theta}\right)$, we have that $\nabla \partial_{t} F \in H^{1}\left(\Theta ; H^{-1}(0, T)\right)$. Therefore, the restriction $F \mapsto \gamma\left(\partial_{t} \nabla F\right)$ on $(0, T) \times \partial \Theta$ is well defined on the space $H^{1 / 2}\left(\partial \Theta ; H^{-1}(0, T)\right)$. Moreover, the fact that $\mathbf{w}=\left(\partial_{2} F,-\partial_{1} F\right) \in Y$ implies $\gamma \partial_{t} \nabla F \in$ $\mathbf{L}^{2}((0, T) \times \partial \Theta)$. Hence

$$
\gamma \partial_{t} \partial_{n} F \in L^{2}((0, T) \times \partial \Theta) \quad \text { and } \quad \gamma \partial_{t} \partial_{\tau} F \in L^{2}((0, T) \times \partial \Theta) .
$$

Relation (4.40) implies that

$$
\gamma \partial_{t} F \in L^{2}\left(0, T ; H^{1}(\partial \Theta)\right) .
$$

By (4.39) and (4.41), we have that

$$
\gamma \Delta F \in L^{1}\left(0, T ; B^{-1 / \alpha, \alpha}(\partial \Theta)\right)
$$

for $1<\alpha<2$. Since

$$
F \in \mathcal{H}^{(2)}\left(Q_{\Theta}\right) \subset L^{2}\left(0, T ; H^{2}(\Theta)\right)
$$

we see that

$$
\gamma F \in L^{2}\left(0, T ; H^{3 / 2}(\partial \Theta)\right), \quad \gamma \partial_{\tau} F \in L^{2}\left(0, T ; H^{1 / 2}(\partial \Theta)\right)
$$

and

$$
\gamma \partial_{n} F \in L^{2}\left(0, T ; H^{1 / 2}(\partial \Theta)\right) .
$$

We claim that

$$
\gamma \partial_{n \tau} F \in L^{2}\left(0, T ; H^{-1 / 2}(\partial \Theta)\right) \quad \text { and } \quad \gamma \partial_{\tau \tau} F \in L^{2}\left(0, T ; H^{-1 / 2}(\partial \Theta)\right) .
$$

To prove this claim, we proceed as follows. We multiply $F$ by a cut-off function with support in a neighborhood of $\partial \Theta$. We assume without loss of generality that $\Theta$ coincides with the half-plane $\mathbb{R}_{+}^{2}=\left\{\left(x_{1}, x_{2}\right): x_{2} \geq 0\right\}$ and $\partial \Theta$ coincides with $\left\{\left(x_{1}, x_{2}\right): x_{2}=0\right\}$. We set $F_{1}=\partial_{\tau} F$ and $F_{2}=\partial_{\tau \tau} F$. From (4.43), we easily deduce that

$$
F_{2} \in L^{2}\left((0, T) \times \mathbb{R}_{+}^{2}\right), \quad \partial_{\tau \tau} F_{2} \in L^{2}\left((0, T) \times \mathbb{R}_{+} ; H^{-2}(\mathbb{R})\right)
$$

and

$$
\Delta F_{2}=\partial_{\tau \tau}\left(\partial_{n n}+\partial_{\tau \tau}\right) F \in L^{2}\left((0, T) \times \mathbb{R}_{+} ; H^{-2}(\mathbb{R})\right) .
$$

These in turn imply

$$
\partial_{n n} F_{2}=\Delta F_{2}-\partial_{\tau \tau} F_{2} \in L^{2}\left((0, T) \times \mathbb{R}_{+} ; H^{-2}(\mathbb{R})\right) .
$$

Relations (4.47)-(4.49) and the trace theorem [21, Chapter 5, section 3] yield the second relation in our claim (4.46). We can similarly prove the first relation in (4.46).

By denoting the unit normal vector by $\mathbf{n}=\left(n_{1}, n_{2}\right)$ and the unit tangential vector by $\boldsymbol{\tau}=\left(n_{2},-n_{1}\right)$, we obtain that

$$
\partial_{1} F=n_{1} \partial_{n} F+n_{2} \partial_{\tau} F, \quad \partial_{2} F=n_{2} \partial_{n} F-n_{1} \partial_{\tau} F,
$$


and

$$
\Delta F=\partial_{n n} F+\partial_{\tau \tau} F+\rho_{1} \partial_{n} F+\rho_{2} \partial_{\tau} F,
$$

where $\rho_{1}$ and $\rho_{2}$ are smooth functions. Expressing $\partial_{n n} F$ by $\Delta F, \partial_{\tau \tau} F, \partial_{n} F$, and $\partial_{\tau} F$ in (4.51) and taking into account (4.42), (4.44)-(4.46), and the imbedding

$$
L^{2}\left(0, T ; H^{-1 / 2}(\partial \Theta)\right) \subset L^{1}\left(0, T ; B^{-1 / \alpha, \alpha}(\partial \Theta)\right), \quad 1<\alpha<2,
$$

we deduce that $\partial_{n n} F$ possesses a trace on $\partial \Theta$ and that the trace satisfies

$$
\gamma \partial_{n n} F \in L^{1}\left(0, T ; B^{-1 / \alpha, \alpha}(\partial \Theta)\right) .
$$

Equations (4.28) and (4.50) yield

$$
\nabla w_{1} \cdot \mathbf{n}=n_{2} \partial_{n n} F-n_{1} \partial_{n \tau} F+\beta_{1} \partial_{n} F+\beta_{2} \partial_{\tau} F
$$

and

$$
\nabla w_{2} \cdot \mathbf{n}=-n_{1} \partial_{n n} F-n_{2} \partial_{n \tau} F+\delta_{1} \partial_{n} F+\delta_{2} \partial_{\tau} F,
$$

where $\beta_{1}, \beta_{2}, \delta_{1}$, and $\delta_{2}$ are smooth functions. These two relations give us the expression for $\nabla \mathbf{w} \cdot \mathbf{n}$ in terms of $\partial_{n n} F, \partial_{n \tau} F, \partial_{n} F$, and $\partial_{\tau} F$. Similarly, we obtain the expression for $(\nabla \mathbf{w})^{T} \cdot \mathbf{n}$ in terms of $\partial_{n n} F, \partial_{n \tau} F, \partial_{n} F$, and $\partial_{\tau} F$ :

$$
\left(\partial_{1} \mathbf{w}\right) \cdot \mathbf{n}=-n_{2} \partial_{\tau \tau} F-n_{1} \partial_{n \tau} F+b_{1} \partial_{n} F+b_{2} \partial_{\tau} F
$$

and

$$
\left(\partial_{2} \mathbf{w}\right) \cdot \mathbf{n}=n_{1} \partial_{\tau \tau} F-n_{2} \partial_{n \tau} F+d_{1} \partial_{n} F+d_{2} \partial_{\tau} F,
$$

where $b_{1}, b_{2}, d_{1}$, and $d_{2}$ are smooth functions. These relations together with (4.44)(4.46) and (4.52) imply the assertions of the theorem.

Now we prove a trace result for the pressure field $p$ that satisfies (4.27).

THEOREM 4.10. Assume $\mathbf{w}$ satisfies the hypotheses of Theorem 4.9 and let $p$ be a scalar field such that $p \in L^{2}\left(0, T ; L_{\mathrm{loc}}^{2}(\Omega)\right), \nabla p \in L^{2}\left(0, T ; \mathbf{H}^{-1}(\Omega)\right)$, and (4.27) holds. Then $p \in L^{1}\left(0, T ; X_{\alpha}(\Theta)\right)$ and the restriction mapping $\gamma:\left.p \mapsto(p \mathbf{n})\right|_{\partial \Omega}$ belongs to $\mathcal{L}\left(L^{1}\left(0, T ; X_{\alpha}(\Theta)\right), L^{1}\left(0, T ; \mathbf{B}^{-1 / \alpha, \alpha}(\partial \Omega)\right)\right)$, where $1<\alpha<2$.

Proof. Taking the divergence of (4.27) and using the divergence-free condition (4.30) for $\mathbf{w}$, we obtain

$$
\Delta p=E,
$$

where $E=-2\left[\left(\partial_{1} w_{1}\right)^{2}+\left(\partial_{1} w_{2}\right)\left(\partial_{2} w_{1}\right)\right]$. Let $\alpha^{\prime}$ be the reciprocal conjugate of $\alpha$, i.e., $(1 / \alpha)+\left(1 / \alpha^{\prime}\right)=1$. Since $\alpha^{\prime}>2$, the imbedding $W^{1, \alpha^{\prime}}(\Theta) \hookrightarrow C(\bar{\Theta})$ is continuous so that

$$
\begin{aligned}
\int_{0}^{T} \int_{\Theta} E(t, \mathbf{x}) \phi(\mathbf{x}) d \mathbf{x} d t \leq 2 \int_{0}^{T}\|\mathbf{w}\|_{\mathbf{V}^{1}(\Theta)}^{2} d t\|\phi\|_{C(\bar{\Theta})} \\
\quad \leq C\|\mathbf{w}\|_{L^{2}\left(0, T ; \mathbf{V}^{1}(\Theta)\right)}^{2}\|\phi\|_{W^{1, \alpha^{\prime}}(\Theta)} \quad \forall \phi \in W^{1, \alpha^{\prime}}(\Theta) .
\end{aligned}
$$

Hence $\Delta p \in L^{1}\left(0, T ; W^{-1, \alpha}(\Theta)\right)$. Also, $p \in L^{2}\left(0, T ; L^{2}(\Theta)\right)$. Hence we conclude that $p \in L^{1}\left(0, T ; X_{\alpha}\right)$ so that the desired result about the trace of $p$ follows from Proposition 4.8 and the fact that $\partial \Omega$ is of class $C^{\infty}$. 
Combining Theorems 4.9 and 4.10, we obtain the following result for the stress vector on the boundary corresponding to admissible solutions.

COROLlary 4.11. Assume that $\mathbf{w}$ and $p$ satisfy the hypotheses of Theorems 4.9 and 4.10 . Then, the stress vector $\left.\left(-p \mathbf{n}+\mu\left(\nabla \mathbf{w}+(\nabla \mathbf{w})^{T}\right) \cdot \mathbf{n}\right)\right|_{(0, T) \times \partial \Omega}$ on the boundary belongs to $L^{1}\left(0, T ; \mathbf{B}^{-1 / \alpha, \alpha}(\partial \Omega)\right)$.

5. The existence of an optimal solution. In this section we prove the existence of an optimal solution for both Problem I and Problem II. We first establish a useful lemma.

LEMMA 5.1. Let $R>0$ be a constant such that $\partial \Omega \subset\{\mathbf{x}:|\mathbf{x}|<R\}$ and define $\Omega_{R}=\Omega \cap\{\mathbf{x}:|\mathbf{x}|<R\}$. Then there exists a positive constant $C$ depending only on $R$ such that

$$
\|\mathbf{u}\|_{\mathbf{H}^{1}\left(\Omega_{R}\right)} \leq C\left(\int_{\Omega_{R}}|D(\mathbf{u})|^{2} d \mathbf{x}+\int_{\partial \Omega}|\mathbf{u}|^{2} d s\right) \quad \forall \mathbf{u} \in \mathbf{H}^{1}\left(\Omega_{R}\right) .
$$

Proof. Assume the lemma is false; then we may choose a sequence $\left\{\mathbf{u}_{n}\right\} \subset \mathbf{H}^{1}\left(\Omega_{R}\right)$ such that $\left\|\mathbf{u}_{n}\right\|_{\mathbf{H}^{1}\left(\Omega_{R}\right)}=1$ and

$$
1>n\left(\int_{\Omega_{R}}\left|D\left(\mathbf{u}_{n}\right)\right|^{2} d \mathbf{x}+\int_{\partial \Omega}\left|\mathbf{u}_{n}\right|^{2} d s\right)
$$

so that

$$
\int_{\Omega_{R}}\left|D\left(\mathbf{u}_{n}\right)\right|^{2} d \mathbf{x} \rightarrow 0 \quad \text { and } \quad \int_{\partial \Omega}\left|\mathbf{u}_{n}\right|^{2} d s \rightarrow 0 \quad \text { as } n \rightarrow \infty ;
$$

i.e., $D\left(\mathbf{u}_{n}\right) \rightarrow \mathbf{O}$ in $\mathbf{L}^{2}\left(\Omega_{R}\right)$ (where $\mathbf{O}$ is the zero tensor) and $\mathbf{u}_{n} \rightarrow \mathbf{u}$ in $\mathbf{L}^{2}(\partial \Omega)$. The fact that $\left\|\mathbf{u}_{n}\right\|_{\mathbf{H}^{1}\left(\Omega_{R}\right)}=1$ implies that there exists a subsequence (still denoted by $\left\{\mathbf{u}_{n}\right\}$ ) such that as $n \rightarrow \infty$,

$$
\mathbf{u}_{n} \rightarrow \mathbf{u} \text { in } \mathbf{H}^{1}\left(\Omega_{R}\right), \quad \mathbf{u}_{n} \rightarrow \mathbf{u} \text { in } \mathbf{L}^{2}\left(\Omega_{R}\right), \quad \text { and } \quad \mathbf{u}_{n} \rightarrow \mathbf{u} \text { in } \mathbf{L}^{2}(\partial \Omega)
$$

for some $\mathbf{u} \in \mathbf{H}^{1}\left(\Omega_{R}\right)$, which in turn implies $D\left(\mathbf{u}_{n}\right) \rightarrow D(\mathbf{u})$ in $\mathbf{L}^{2}\left(\Omega_{R}\right)$. Hence we have $D(\mathbf{u})=\mathbf{O}$ in $\mathbf{L}^{2}\left(\Omega_{R}\right)$ and $\mathbf{u}=\mathbf{0}$ in $\mathbf{L}^{2}(\partial \Omega)$, i.e., $D(\mathbf{u}) \equiv \mathbf{O}$ in $\Omega_{R}$ and $\mathbf{u} \equiv \mathbf{0}$ on $\partial \Omega$. Hence $\mathbf{u}$ is a rigid-body motion which can be expressed in the form $\mathbf{u}=\mathbf{a}+\mathbf{b} \times \mathbf{x}$ for all $\mathbf{x} \in \Omega_{R}$, where $\mathbf{a}$ and $\mathbf{b}$ are constant vectors (see [22] or [23]). $\mathbf{u}$ being a linear function and $\mathbf{u} \equiv \mathbf{0}$ on $\partial \Omega$ easily leads us to $\mathbf{a}=\mathbf{0}$ and $\mathbf{b}=\mathbf{0}$, i.e., $\mathbf{u} \equiv \mathbf{0}$ in $\Omega_{R}$. On the other hand, we deduce from (5.1) and (5.2) that $D\left(\mathbf{u}_{n}\right) \rightarrow D(\mathbf{u})$ in $\mathbf{L}^{2}\left(\Omega_{R}\right)$ and $\mathbf{u}_{n} \rightarrow \mathbf{u}$ in $\mathbf{L}^{2}\left(\Omega_{R}\right)$. Then, using Korn's second inequality (see, e.g., [22, p. 31]),

$$
\int_{\Omega_{R}}\left(|D(\mathbf{z})|^{2}+|\mathbf{z}|^{2}\right) d \mathbf{x} \geq C\|\mathbf{z}\|_{\mathbf{H}^{1}\left(\Omega_{R}\right)}^{2} \quad \forall \mathbf{z} \in \mathbf{H}^{1}\left(\Omega_{R}\right),
$$

we conclude $\mathbf{u}_{n} \rightarrow \mathbf{u}$ in $\mathbf{H}^{1}\left(\Omega_{R}\right)$ so that $\|\mathbf{u}\|_{\mathbf{H}^{1}\left(\Omega_{R}\right)}=\lim _{n \rightarrow \infty}\left\|\mathbf{u}_{n}\right\|_{\mathbf{H}^{1}\left(\Omega_{R}\right)}=1$, i.e., $\mathbf{u} \neq \mathbf{0}$. This gives a contradiction. Hence the lemma is proved.

As a consequence of Lemma 5.1, we obtain the following.

COROLlaRY 5.2. There exists a constant $C>0$ depending only on $\Omega$ such that

$$
\|\mathbf{u}\|_{\mathbf{H}^{1 / 2}(\partial \Omega)} \leq C\left(\int_{\Omega}|D(\mathbf{u})|^{2} d \mathbf{x}+\int_{\partial \Omega}|\mathbf{u}|^{2} d s\right) \quad \forall \mathbf{u} \in \mathbf{H}^{1}(\Omega) .
$$

Proof. We fix an $R>0$ such that $\partial \Omega \subset\{\mathbf{x}:|\mathbf{x}|<R\}$ ( $R$ is determined, albeit not uniquely, by $\Omega$ ). Then by Lemma 5.1 and the trace theorem for $\mathbf{H}^{1}\left(\Omega_{R}\right)$, there 
exists a constant $C>0$ (depending only on $R$ and $\Omega$ ) such that

$$
\|\mathbf{u}\|_{\mathbf{H}^{1 / 2}(\partial \Omega)} \leq C\left(\int_{\Omega_{R}}|D(\mathbf{u})|^{2} d \mathbf{x}+\int_{\partial \Omega}|\mathbf{u}|^{2} d s\right) \quad \forall \mathbf{u} \in \mathbf{H}^{1}\left(\Omega_{R}\right) .
$$

Thus the desired estimate follows from the last inequality and the fact that $\Omega_{R} \subset \Omega . \quad \square$

THEOREM 5.3. There exists a solution $\mathbf{w} \in Y$ for Problem $\mathbf{I}$; there exists a solution $\mathbf{w} \in Y$ for Problem II.

Proof. The proofs for Problem I and Problem II are essentially the same, and we will only consider Problem I. Theorem 4.4 guarantees that the admissible set $\mathcal{V}_{a d}$ is nonempty; indeed, we choose a boundary data in $\mathbf{C}^{\infty}([0, T] \times \partial \Omega)$, and then by Theorem 4.4, there exists a solution in $\mathcal{V}^{(1)}\left(Q_{T}\right)$ for the Navier-Stokes equations satisfying this chosen smooth boundary data. This solution clearly belongs to $\mathcal{V}_{a d}$; i.e., $\mathcal{V}_{a d}$; is nonempty. It is easy to verify that $\mathcal{J}_{N}(\cdot)$ is bounded from below in $Y$. Thus, we may choose a sequence $\left\{\mathbf{w}_{n}\right\} \subset \mathcal{V}_{a d}$ such that

$$
\begin{gathered}
\lim _{n \rightarrow \infty} \mathcal{J}_{N}\left(\mathbf{w}_{n}\right)=\inf _{\mathbf{w} \in \mathcal{V}_{a d}} \mathcal{J}_{N}(\mathbf{w}), \\
\left\langle\partial_{t} \mathbf{w}_{n}, \mathbf{z}\right\rangle+\mu \int_{\Omega} \nabla \mathbf{w}_{n}: \nabla \mathbf{z} d \mathbf{x}+\int_{\Omega}\left(\left[\mathbf{w}_{n}+\mathbf{v}_{\infty}\right] \cdot \nabla\right) \mathbf{w}_{n} \cdot \mathbf{z} d \mathbf{x} \\
=0 \quad \forall \mathbf{z} \in \mathbf{V}_{0}^{1}(\Omega), \text { a.e. } t \in(0, T),
\end{gathered}
$$

and

$$
\left.\mathbf{w}_{n}\right|_{t=0}=\mathbf{w}_{0} \quad \text { in } \mathbf{V}^{0}(\Omega) .
$$

Using (3.4) and the conditions on $k$ and $N$ given in the definition of Problem I, we obtain that

$$
\mu \int_{0}^{T} \int_{\Omega} \mathcal{D}\left(\mathbf{w}_{n}\right): \mathcal{D}\left(\mathbf{w}_{n}\right) d \mathbf{x} d t+\int_{0}^{T} \int_{\partial \Omega}\left(\left|\partial_{t} \mathbf{w}_{n}\right|^{2}+\left|\mathbf{w}_{n}\right|^{k}\right) d s d t \leq C .
$$

The last inequality and Corollary 5.2 imply that

$$
\left\|\mathbf{w}_{n}\right\|_{L^{k}((0, T) \times \partial \Omega)}+\left\|\mathbf{w}_{n}\right\|_{H^{1}\left(0, T ; \mathbf{L}^{2}(\partial \Omega)\right)}+\left\|\mathbf{w}_{n}\right\|_{L^{2}\left(0, T ; \mathbf{H}^{1 / 2}(\partial \Omega)\right)} \leq C .
$$

Thus, the estimate of Theorem 4.4 with (5.5) gives us the bound

$$
\left\|\partial_{t} \mathbf{w}_{n}\right\|_{L^{2}\left(0, T ; \mathbf{V}^{-1}(\Omega)\right)}+\left\|\mathbf{w}_{n}\right\|_{L^{2}\left(0, T ; \mathbf{V}^{1}(\Omega)\right)} \leq C,
$$

which allows us to choose a weakly convergent subsequence

$$
\mathbf{w}_{n} \rightarrow \widehat{\mathbf{w}} \text { in } L^{2}\left(0, T ; \mathbf{V}^{1}(\Omega)\right)
$$

and

$$
\partial_{t} \mathbf{w}_{n} \rightarrow \partial_{t} \widehat{\mathbf{w}} \quad \text { in } L^{2}\left(0, T ; \mathbf{V}^{-1}(\Omega)\right)
$$

for some $\widehat{\mathbf{w}} \in \mathcal{V}^{(1)}\left(Q_{T}\right)$. For each $R>0$ we let $B_{R}=\left\{\mathbf{x} \in \mathbb{R}^{2}:|\mathbf{x}|<R\right\}$. Since the space

$$
\mathcal{V}_{R}^{(1)}\left(\Omega \cap B_{R}\right) \equiv\left\{\mathbf{u} \in L^{2}\left(0, T ; \mathbf{V}^{1}\left(\Omega \cap B_{R}\right)\right): \partial_{t} \mathbf{u} \in L^{2}\left(0, T ; \mathbf{V}^{-1}\left(\Omega \cap B_{R}\right)\right)\right\}
$$


equipped with the norm $\|\mathbf{u}\|_{\mathcal{V}_{R}^{(1)}\left(\Omega \cap B_{R}\right)}^{2}=\|\mathbf{u}\|_{L^{2}\left(0, T ; \mathbf{V}^{1}\left(\Omega \cap B_{R}\right)\right)}^{2}+\left\|\partial_{t} \mathbf{u}\right\|_{L^{2}\left(0, T ; \mathbf{V}^{-1}\left(\Omega \cap B_{R}\right)\right)}^{2}$ is compactly imbedded into $\mathbf{L}^{2}\left((0, T) \times\left(\Omega \cap B_{R}\right)\right)$, we may use (5.6) and (5.7) to conclude that

$$
\mathbf{w}_{n} \rightarrow \widehat{\mathbf{w}} \quad \text { in } \mathbf{L}^{2}\left((0, T) \times\left(\Omega \cap B_{R}\right)\right) .
$$

For each arbitrarily given $\mathbf{z} \in \mathbf{C}_{0}^{\infty}(\Omega) \cap \mathbf{V}_{0}^{1}(\Omega)$, relations (5.6)-(5.8) allow us to pass to the limit in (5.3) to deduce that

$$
\begin{array}{r}
\left\langle\partial_{t} \widehat{\mathbf{w}}(t), \mathbf{z}\right\rangle+\mu \int_{\Omega} \nabla \widehat{\mathbf{w}}(t): \nabla \mathbf{z} d \mathbf{x}+\int_{\Omega}\left(\left[\widehat{\mathbf{w}}(t)+\mathbf{v}_{\infty}\right] \cdot \nabla\right) \widehat{\mathbf{w}}(t) \cdot \mathbf{z} d \mathbf{x} \\
=0 \quad \text { a.e. } t \in(0, T) .
\end{array}
$$

Then, using the denseness of $\mathbf{C}_{0}^{\infty}(\Omega) \cap \mathbf{V}_{0}^{1}(\Omega)$ in $\mathbf{V}_{0}^{1}(\Omega)$, we obtain

$$
\begin{array}{r}
\left\langle\partial_{t} \widehat{\mathbf{w}}(t), \mathbf{z}\right\rangle+\mu \int_{\Omega} \nabla \widehat{\mathbf{w}}(t): \nabla \mathbf{z} d \mathbf{x}+\int_{\Omega}\left(\left[\widehat{\mathbf{w}}(t)+\mathbf{v}_{\infty}\right] \cdot \nabla\right) \widehat{\mathbf{w}}(t) \cdot \mathbf{z} d \mathbf{x} \\
=0 \quad \forall \mathbf{z} \in \mathbf{V}_{0}^{1}(\Omega), \quad \text { a.e. } t \in(0, T) ;
\end{array}
$$

i.e., $\widehat{\mathbf{w}}$ satisfies the weak form of the Navier-Stokes equations.

Relations (5.6) and trace theorems imply

$$
\mathbf{w}_{n} \rightarrow \widehat{\mathbf{w}} \quad \text { in } L^{2}\left(0, T ; \mathbf{H}^{1 / 2}(\partial \Omega)\right)
$$

so that

$$
\mathbf{w}_{n} \rightarrow \widehat{\mathbf{w}} \quad \text { in } \mathbf{L}^{2}((0, T) \times \partial \Omega) .
$$

The estimate (5.5) implies

$$
\mathbf{w}_{n} \rightarrow \mathbf{h} \quad \text { in } \mathbf{L}^{k}((0, T) \times \partial \Omega) \cap H^{1}\left(0, T ; \mathbf{L}^{2}(\partial \Omega)\right) \cap L^{2}\left(0, T ; \mathbf{H}^{1 / 2}(\partial \Omega)\right)
$$

for some $\mathbf{h} \in \mathbf{L}^{k}((0, T) \times \partial \Omega) \cap H^{1}\left(0, T ; \mathbf{L}^{2}(\partial \Omega)\right) \cap L^{2}\left(0, T ; \mathbf{H}^{1 / 2}(\partial \Omega)\right)$. Hence we deduce that $\mathbf{h}=\widehat{\mathbf{w}}$ on $(0, T) \times \partial \Omega$ so that

$$
\mathbf{w}_{n} \rightarrow \widehat{\mathbf{w}} \quad \text { in } \mathbf{L}^{k}((0, T) \times \partial \Omega) \cap H^{1}\left(0, T ; \mathbf{L}^{2}(\partial \Omega)\right) \cap L^{2}\left(0, T ; \mathbf{H}^{1 / 2}(\partial \Omega)\right) .
$$

Thus, we have shown that $\widehat{\mathbf{w}} \in Y$. The continuous imbedding of $\mathcal{V}^{(1)}\left(Q_{T}\right)$ into $\mathbf{C}\left([0, T] ; \mathbf{V}^{\mathbf{0}}\right)$ (see Lemma 3.1) yields that for each $\tau \in[0, T]$, the trace operator $\left.\mathbf{w} \mapsto \mathbf{w}\right|_{t=\tau}$ is bounded from $\mathcal{V}^{(1)}\left(Q_{T}\right)$ into $\mathbf{V}^{0}(\Omega)$. Hence, using the weak convergence $\mathbf{w}_{n} \rightarrow \widehat{\mathbf{w}}$ in $\mathcal{V}^{(1)}\left(Q_{T}\right)$ and the fact that bounded linear operators preserve weak convergence we obtain

$$
\mathbf{w}_{0}=\left.\left.\mathbf{w}_{n}\right|_{t=0} \rightarrow \widehat{\mathbf{w}}\right|_{t=0} \quad \text { in } \mathbf{V}^{0}(\Omega)
$$

and

$$
\left.\left.\mathbf{w}_{n}\right|_{t=T} \rightarrow \widehat{\mathbf{w}}\right|_{t=T} \quad \text { in } \mathbf{V}^{0}(\Omega) .
$$

Now, we pass to the limit in the functional $\mathcal{J}_{N}$. We first examine the term $\int_{0}^{T} \int_{\partial \Omega}|\mathbf{w}|^{2} \mathbf{w} \cdot \mathbf{n} d s d t$ in the functional. By the compact imbedding result (see [1])

$$
\begin{aligned}
& \mathbf{L}^{k}((0, T) \times \partial \Omega) \cap H^{1}\left(0, T ; \mathbf{L}^{2}(\partial \Omega)\right) \cap L^{2}\left(0, T ; \mathbf{H}^{1 / 2}(\partial \Omega)\right) \\
& \hookrightarrow \mathbf{H}^{1 / 2}((0, T) \times \partial \Omega) \hookrightarrow \hookrightarrow \mathbf{L}^{3}((0, T) \times \partial \Omega),
\end{aligned}
$$


we obtain from (5.10) that

$$
\left.\left.\mathbf{w}_{n}\right|_{\partial \Omega} \rightarrow \widehat{\mathbf{w}}\right|_{\partial \Omega} \quad \text { in } \mathbf{L}^{3}((0, T) \times \partial \Omega)
$$

so that

$$
\lim _{n \rightarrow \infty} \int_{0}^{T} \int_{\partial \Omega}\left|\mathbf{w}_{n}\right|^{2} \mathbf{w}_{n} \cdot \mathbf{n} d s d t=\int_{0}^{T} \int_{\partial \Omega}|\widehat{\mathbf{w}}|^{2} \hat{\mathbf{w}} \cdot \mathbf{n} d s d t
$$

All the remaining terms in the functional $\mathcal{J}_{N}$ are sequentially weakly lower semicontinuous; thus, using the weak convergence results obtained earlier, we have that

$$
\mathcal{J}_{N}(\widehat{\mathbf{w}}) \leq \liminf _{n \rightarrow \infty} \mathcal{J}_{N}\left(\mathbf{w}_{n}\right) .
$$

Hence, we have shown that $\widehat{\mathbf{w}} \in Y$ is indeed a solution to Problem I.

REMARK. The proof of Theorem 5.2 for Problem II can proceed first by substituting $w=w_{n}$ into (3.5) and (3.6) to obtain the estimate (5.4) and then passing to the limit as $n \rightarrow \infty$.

REMARK. Since the optimal solution is sought in the space $Y$ whose boundary values are more regular than the trace of $\mathcal{V}^{(1)}\left(Q_{T}\right)$, we expect the optimal solution to be more regular than merely in $\mathcal{V}^{(1)}\left(Q_{T}\right)$.

REMARK. The result also holds for many other cost functionals such as the $L^{2}$ norm of the vorticity functional used in [2] or the velocity matching functional

$$
\mathcal{K}(\mathbf{w})=\frac{1}{2} \int_{0}^{T} \int_{\Omega}\left|\mathbf{w}-\mathbf{w}_{0}\right|^{2} d \mathbf{x} d t+\frac{1}{2} \int_{0}^{T} \int_{\partial \Omega}\left(\left|\partial_{t} \mathbf{w}\right|^{2}+|\mathbf{w}|^{2}+\left|\nabla_{s} \mathbf{w}\right|^{2}\right) d s d t,
$$

where $\nabla_{s}$ denotes the surface gradient on $\partial \Omega$. Using similar arguments we may, for example, conclude that there exists a $\widehat{\mathbf{w}} \in \mathcal{V}_{a d}$ such that $\mathcal{K}(\widehat{\mathbf{w}})=\inf _{\mathbf{w} \in \mathcal{V}_{a d}} \mathcal{K}(\mathbf{w})$. 口

6. The optimality system. Having proved that an optimal solution $\mathbf{w}$ exists, we now use Lagrange multiplier principles to characterize the optimal solution; i.e., we obtain an optimality system of partial differential equations that the optimal solution w and Lagrange multipliers must satisfy. This optimality system can serve as the basis for computing approximations to optimal solutions numerically.

6.1. Abstract Lagrange multiplier principles. We consider an abstract minimization problem. Let $X_{1}$ and $X_{2}$ be two Banach spaces. Let $f: X_{1} \rightarrow \mathbb{R}$ and $g_{j}: X_{1} \rightarrow \mathbb{R}$ be functionals and $F: X_{1} \rightarrow X_{2}$ be a mapping. We seek a $w \in X_{1}$ such that

$$
f(w)=\inf _{u \in \mathcal{W}_{a d}} f(u)
$$

where

$$
\mathcal{W}_{a d}=\left\{u \in X_{1}: F(u)=0 \text {, and } g_{j}(u) \leq 0 \text { for } j=1, \ldots, m\right\} .
$$

The Lagrange functional for the minimization problem (6.1) is defined by

$$
\mathcal{L}(w, \boldsymbol{\lambda}, q)=\lambda_{0} f(w)+\langle F(w), q\rangle+\sum_{i=1}^{m} \lambda_{j} g_{j}(w)
$$


for all $w \in X_{1}, \boldsymbol{\lambda}=\left(\lambda_{0}, \lambda_{1}, \ldots, \lambda_{m}\right)^{T} \in \mathbb{R}^{m+1}$, and $q \in X_{2}^{*}$. We quote a standard abstract Lagrange principle in the following particular form (see [3]).

THEOREM 6.1. Let $w$ be a solution of (6.1). Assume that the mappings $f, g_{j}$, and $F$ are continuously differentiable and that the image of the operator $F^{\prime}(w): X_{1} \rightarrow X_{2}$ is closed. Then there exists $a q \in X_{2}^{*}$ and $a \boldsymbol{\lambda}=\left(\lambda_{0}, \lambda_{1}, \ldots, \lambda_{m}\right)^{T} \in \mathbb{R}^{m+1}$ such that the pair $(q, \boldsymbol{\lambda}) \neq(0, \mathbf{0})$,

$$
\begin{gathered}
\left\langle\mathcal{L}_{w}(w, \boldsymbol{\lambda}, q), h\right\rangle=0 \quad \forall h \in X_{1}, \\
\lambda_{j} \geq 0, \quad j=0,1, \ldots, m, \quad \text { and } \quad \lambda_{j} g_{j}(w)=0, \quad j=1, \ldots, m,
\end{gathered}
$$

where $\mathcal{L}_{w}(\cdot, \cdot, \cdot)$ denotes the Fréchet derivative of $\mathcal{L}$ with respect to the first argument. Furthermore, if $F^{\prime}(w): X_{1} \rightarrow X_{2}$ is an epimorphism and the constraints $g_{i}(w) \leq 0$ are absent in problem (6.1), then $\lambda_{0} \neq 0$ and $\lambda_{0}$ can be taken as 1 .

6.2. The weak form of an optimality system. Now we apply the abstract Lagrange principle to Problem I and Problem II to obtain an optimality system of equations for each case. We first examine Problem I. We first derive the adjoint equation, in the weak form, for the optimal control problem.

THEOREM 6.2. Assume $\mathbf{w} \in \mathcal{V}^{(1)}\left(Q_{T}\right)$ is a solution for Problem I. Then there exists a $\mathbf{q} \in \mathcal{V}^{(1)}\left(Q_{T}\right) \cap L^{2}\left(0, T ; \mathbf{V}_{0}^{1}(\Omega)\right)$ such that

$$
\begin{aligned}
& 2 \mu \int_{0}^{T} \int_{\Omega} \mathcal{D}(\mathbf{w}): \mathcal{D}(\mathbf{h}) d \mathbf{x} d t+2 \mu \int_{0}^{T} \int_{\Omega} \mathcal{D}(\mathbf{h}): \mathcal{D}(\mathbf{q}) d \mathbf{x} d t \\
& \quad+\int_{0}^{T} \int_{\Omega}\left\{(\mathbf{h} \cdot \nabla) \mathbf{w}+(\mathbf{w} \cdot \nabla) \mathbf{h}+\left(\mathbf{v}_{\infty} \cdot \nabla\right) \mathbf{h}\right\} \cdot \mathbf{q} d \mathbf{x} d t \\
& \quad+\int_{0}^{T}\left\langle\partial_{t} \mathbf{h}(t, \cdot), \mathbf{q}(t, \cdot)\right\rangle d t \\
& \quad+N\left(\int_{0}^{T} \int_{\partial \Omega} 2 \partial_{t} \mathbf{w} \cdot \partial_{t} \mathbf{h} d s d t+k \int_{0}^{T} \int_{\partial \Omega}\left|\mathbf{w}+\mathbf{v}_{\infty}\right|^{k-2}\left(\mathbf{w}+\mathbf{v}_{\infty}\right) \cdot \mathbf{h} d s d t\right) \\
& \quad+\frac{1}{2} \int_{0}^{T} \int_{\partial \Omega}\left\{\left(\mathbf{w}+\mathbf{v}_{\infty}\right) \cdot \mathbf{n}(\mathbf{w} \cdot \mathbf{h})+\frac{1}{2}(\mathbf{h} \cdot \mathbf{n})|\mathbf{w}|^{2}\right\} d s d t \\
& \quad+\frac{1}{2} \int_{\Omega} \mathbf{w}(T, \mathbf{x}) \cdot \mathbf{h}(T, \mathbf{x}) d \mathbf{x}=0 \quad \forall \mathbf{h} \in Y_{0},
\end{aligned}
$$

where $Y_{0} \equiv\left\{\mathbf{y} \in Y:\left.\mathbf{y}\right|_{t=0}=\mathbf{0}\right\}$.

Proof. We use the Lagrange multiplier principle (Theorem 6.1) to prove the desired result. We set $X_{1}=Y_{0}$ and $X_{2}=L^{2}\left(0, T ; \mathbf{V}^{-1}(\Omega)\right)$. We define the mappings $f: X_{1} \rightarrow \mathbb{R}$ and $F: X_{1} \rightarrow X_{2}$ as follows:

$$
f(\mathbf{y})=\mathcal{J}_{N}(\mathbf{w}+\mathbf{y})
$$

and

$$
F(\mathbf{y})=\partial_{t}(\mathbf{w}+\mathbf{y})-\mu P \Delta(\mathbf{w}+\mathbf{y})+P\left[\left(\left(\mathbf{w}+\mathbf{y}+\mathbf{v}_{\infty}\right) \cdot \nabla\right)(\mathbf{w}+\mathbf{y})\right],
$$

where $P: \mathbf{H}^{-1}(\Omega) \rightarrow \mathbf{V}^{-1}(\Omega)$ is the projection operator. Constraints $g_{i} \leq 0$ are absent in Problem I. Then $\mathbf{y}=\mathbf{0}$ is the solution of the corresponding extremal problem and $F^{\prime}(\mathbf{0}): X_{1} \rightarrow X_{2}$ is defined by

$$
\left\langle F^{\prime}(\mathbf{0}), \mathbf{y}\right\rangle=\partial_{t} \mathbf{y}-\mu P \Delta \mathbf{y}+P\left[(\mathbf{y} \cdot \nabla) \mathbf{w}+\left(\left(\mathbf{w}+\mathbf{v}_{\infty}\right) \cdot \nabla\right) \mathbf{y}\right] .
$$


To show that $F^{\prime}(\mathbf{0})$ is an epimorphism, we first observe that this operator is continuous. Next we need to show that for each $\mathbf{f} \in L^{2}\left(0, T ; \mathbf{V}^{-1}(\Omega)\right)$ the system $(6.5)$

$$
\begin{aligned}
& \left\langle\partial_{t} \mathbf{y}(t), \mathbf{z}\right\rangle+\mu \int_{\Omega} \nabla \mathbf{y}(t): \nabla \mathbf{z} d \mathbf{x}+\int_{\Omega}\left(\left(\mathbf{w}(t)+\mathbf{v}_{\infty}\right) \cdot \nabla\right) \mathbf{y}(t) \cdot \mathbf{z} d \mathbf{x} \\
& \quad+\int_{\Omega}(\mathbf{y}(t) \cdot \nabla) \mathbf{w}(t) \cdot \mathbf{z} d \mathbf{x}=\int_{\Omega} \mathbf{f}(t) \cdot \mathbf{z} d \mathbf{x} \quad \forall \mathbf{z} \in \mathbf{V}_{0}^{1}(\Omega), \text { a.e. } t \in(0, T),
\end{aligned}
$$

and

$$
\left.\mathbf{y}\right|_{t=0}=\mathbf{0} \quad \text { in } \mathbf{V}^{0}(\Omega)
$$

has a solution $\mathbf{y} \in Y_{0}$. We supplement this system with the boundary condition

$$
\left.\mathbf{y}\right|_{(0, T) \times \partial \Omega}=\mathbf{0} .
$$

Using the techniques in the proof of Theorem 4.4 we see that (6.5)-(6.7) indeed has a (unique) solution $\mathbf{y} \in \mathcal{V}^{(1)}\left(Q_{T}\right)$. (The situation now is even simpler, as the system (6.5)-(6.7) is linear.) Clearly, $\mathbf{y} \in Y_{0}$. Hence, we have verified all the assumptions in Theorem 6.1 and we conclude that there exists a $\mathbf{q} \in X_{2}^{*}=L^{2}\left(0, T ; \mathbf{V}_{0}^{1}(\Omega)\right)$ such that

$$
\left.\left\langle\mathcal{L}_{\mathbf{y}}(\mathbf{y}, \mathbf{q}), \mathbf{h}\right\rangle\right|_{y=0}=0 \quad \forall \mathbf{h} \in Y_{0},
$$

where the Lagrange functional for Problem I is defined by

$$
\begin{aligned}
\mathcal{L}(\mathbf{y}, \mathbf{q})= & \mu \int_{0}^{T} \int_{\Omega}|\mathcal{D}(\mathbf{w}+\mathbf{y})|^{2} d \mathbf{x} d t+\frac{1}{4} \int_{\Omega}|\mathbf{w}(T, \mathbf{x})+\mathbf{y}(T, \mathbf{x})|^{2} d \mathbf{x} \\
& +\frac{1}{4} \int_{0}^{T} \int_{\partial \Omega}\left(\mathbf{w}+\mathbf{y}+\mathbf{v}_{\infty}\right) \cdot \mathbf{n}|\mathbf{w}+\mathbf{y}|^{2} d s d t \\
& +\int_{0}^{T} \int_{\Omega} \partial_{t}(\mathbf{w}+\mathbf{y}) \cdot \mathbf{q} d \mathbf{x} d t+2 \mu \int_{0}^{T} \int_{\Omega} \mathcal{D}(\mathbf{w}+\mathbf{y}): \mathcal{D}(\mathbf{q}) d \mathbf{x} d t \\
& +\int_{0}^{T} \int_{\Omega}\left\{[(\mathbf{w}+\mathbf{y}) \cdot \nabla](\mathbf{w}+\mathbf{y})+\left(\mathbf{v}_{\infty} \cdot \nabla\right)(\mathbf{w}+\mathbf{y})\right\} \cdot \mathbf{q} d \mathbf{x} d t \\
& +N\left(\int_{0}^{T} \int_{\partial \Omega}\left|\partial_{t} \mathbf{w}+\partial_{t} \mathbf{y}\right|^{2} d s d t+\int_{0}^{T} \int_{\partial \Omega}\left|\mathbf{w}+\mathbf{y}+\mathbf{v}_{\infty}\right|^{k} d s d t\right)
\end{aligned}
$$

for all $\mathbf{y} \in X_{1}$ and $\mathbf{q} \in X_{2}^{*}=L^{2}\left(0, T ; \mathbf{V}_{0}^{1}(\Omega)\right.$ ). (Note that we have chosen $\lambda_{0}=1$ in the definition (6.9); this is justified by Theorem 6.1 and the fact that $F^{\prime}(\mathbf{0})$ is an epimorphism.) Substituting (6.9) into (6.8) we obtain (6.4). By varying $\mathbf{h}$ in $\mathcal{E}=\left\{\mathbf{v} \in \mathbf{C}_{0}^{\infty}((0, T) \times \Omega): \operatorname{div} \mathbf{v}=0\right\} \subset Y_{0}$, we obtain in the sense of distributions defined on solenoidal vector fields:

$$
-\partial_{t} \mathbf{q}-\mu \Delta \mathbf{q}+\mathbf{q} \cdot(\nabla \mathbf{w})^{T}-(\mathbf{w} \cdot \nabla) \mathbf{q}-\left(\mathbf{v}_{\infty} \cdot \nabla\right) \mathbf{q}=\mu \Delta \mathbf{w} \quad \text { in } \mathcal{E}^{\prime},
$$

or equivalently,

$$
-\partial_{t} \mathbf{q}=\mu \Delta \mathbf{q}-\mathbf{q} \cdot(\nabla \mathbf{w})^{T}+(\mathbf{w} \cdot \nabla) \mathbf{q}+\left(\mathbf{v}_{\infty} \cdot \nabla\right) \mathbf{q}+\mu \Delta \mathbf{w} \quad \text { in } \mathcal{E}^{\prime} .
$$

From the fact that $\mathbf{w} \in \mathcal{V}^{(1)}\left(Q_{T}\right)$ and $\mathbf{q} \in L^{2}\left(0, T ; \mathbf{V}_{0}^{1}(\Omega)\right)$, we easily deduce $\partial_{t} \mathbf{q} \in$ $L^{2}\left(0, T ; \mathbf{V}^{-1}(\Omega)\right)$. Hence, we have proved $\mathbf{q} \in \mathcal{V}^{(1)}\left(Q_{T}\right)$. 
6.3. Green's formulae. To interpret the weak optimality system (6.4) as a system of partial differential equations with boundary conditions, we will need some Green's formulae for the optimal solution $\mathbf{w}$, the Lagrange multiplier $\mathbf{q}$, and their associated pressure fields $p$ and $r$, respectively.

We note that if $\mathbf{q}$ is a solution of (6.4) or (6.10), then by De Rham's lemma (see $[14]$ and $[28])$, there exists an $\widetilde{r} \in L^{2}\left(0, T ; L_{\mathrm{loc}}^{2}(\Omega)\right)$ such that $\nabla \widetilde{r} \in L^{2}\left(0, T ; \mathbf{H}^{-1}(\Omega)\right)$ and

$$
-\partial_{t} \mathbf{q}-\mu \Delta \mathbf{q}+\mathbf{q} \cdot(\nabla \mathbf{w})^{T}-(\mathbf{w} \cdot \nabla) \mathbf{q}-\left(\mathbf{v}_{\infty} \cdot \nabla\right) \mathbf{q}+\nabla \widetilde{r}=\mu \Delta \mathbf{w}
$$

in the sense of distributions. Through the change of variable $r=\widetilde{r}+p$, where $p$ satisfies (4.27), we see that $r \in L^{2}\left(0, T ; L_{\mathrm{loc}}^{2}(\Omega)\right), \nabla r \in L^{2}\left(0, T ; \mathbf{H}^{-1}(\Omega)\right)$, and

$$
-\partial_{t} \mathbf{q}-\mu \Delta \mathbf{q}+\mathbf{q} \cdot(\nabla \mathbf{w})^{T}-(\mathbf{w} \cdot \nabla) \mathbf{q}-\left(\mathbf{v}_{\infty} \cdot \nabla\right) \mathbf{q}+\nabla r=\mu \Delta \mathbf{w}-\nabla p
$$

in the sense of distributions. We now prove the trace theorems for $\left((\nabla \mathbf{q})+(\nabla \mathbf{q})^{T}\right) \cdot \mathbf{n}$ and $r$ as was done for $\left((\nabla \mathbf{w})+(\nabla \mathbf{w})^{T}\right) \cdot \mathbf{n}$ and $p$ in section 4.3 ; we will also derive some Green's formulae that are useful in interpreting the weak optimality system as a boundary value problem for a system of partial differential equations.

LEMMA 6.3. Assume $\mathbf{w}$ is a solution for Problem I and let $\mathbf{q} \in \mathcal{V}^{(1)}\left(Q_{T}\right) \cap$ $L^{2}\left(0, T ; \mathbf{V}_{0}^{1}(\Omega)\right)$ be a solution of (6.4). Let $r \in L^{2}\left(0, T ; L_{\text {loc }}^{2}(\Omega)\right)$ satisfy (6.12) and $\nabla r \in L^{2}\left(0, T ; \mathbf{H}^{-1}(\Omega)\right)$. Then

$$
\begin{gathered}
\gamma[(\nabla \mathbf{q}) \cdot \mathbf{n}] \in L^{1}\left(0, T ; \mathbf{B}^{-1 / \alpha, \alpha}(\partial \Omega)\right), \gamma\left[(\nabla \mathbf{q})^{T} \cdot \mathbf{n}\right] \in L^{1}\left(0, T ; \mathbf{B}^{-1 / \alpha, \alpha}(\partial \Omega)\right) \\
\gamma(r \mathbf{n}) \in L^{1}\left(0, T ; \mathbf{B}^{-1 / \alpha, \alpha}(\partial \Omega)\right)
\end{gathered}
$$

and therefore

$$
\gamma\left[\left(-r I+(\nabla \mathbf{q})+(\nabla \mathbf{q})^{T}\right) \cdot \mathbf{n} \in L^{1}\left(0, T ; \mathbf{B}^{-1 / \alpha, \alpha}(\partial \Omega)\right)\right],
$$

where $1<\alpha<2$.

Proof. As in section 4.3, we introduce the bounded subdomain $\Theta \subset \Omega$ such that $\partial \Omega \subset \partial \Theta$. We introduce on $\Theta$ the streamfunction $E$ for $\mathbf{q}=\left(q_{1}, q_{2}\right)$ such that

$$
q_{1}=\partial_{2} E \quad \text { and } \quad q_{2}=-\partial_{1} E .
$$

Then, by applying the curl operator to (6.11), we obtain that

$$
\Delta\left(\partial_{t} E+\mu E+\mu \Delta F\right)=G_{1},
$$

where $F$ is the streamfunction for $\mathbf{w}$ introduced in (4.28) and

$G_{1}=2\left(\partial_{1} w_{1}\right)\left(\partial_{2} q_{1}+\partial_{1} q_{2}\right)+2\left(\partial_{2} q_{2}\right)\left(\partial_{1} w_{2}+\partial_{2} w_{1}\right)+\left(w_{1}+v_{\infty, 1}\right) \Delta q_{2}-\left(w_{2}+v_{\infty, 2}\right) \Delta q_{1}$.

Also, the fact that $\left.\mathbf{q}\right|_{S_{T}}=\mathbf{0}$ allows us to choose $E$ to satisfy $\left.E\right|_{S_{T}}=0$. Analogous to the proof of Lemma 4.5, we obtain

$$
G_{1} \in L^{1}\left(0, T ; W^{-1, \alpha}(\Theta)\right), \quad 1<\alpha<2 .
$$

Since $\mathbf{q} \in \mathcal{V}^{(1)}\left(Q_{T}\right)$ we have $E \in \mathcal{H}^{(2)}\left(Q_{T}\right)$. Thus, (6.16) and (6.17) yield

$$
\partial_{t} E+\mu \Delta E+\mu \Delta F \in L^{1}\left(0, T ; X_{\alpha}\right), \quad 1<\alpha<2 .
$$


By virtue of Proposition 4.8 we obtain

$$
\gamma\left(\partial_{t} E+\mu \Delta E+\mu \Delta F\right) \in L^{1}\left(0, T ; B^{-1 / \alpha, \alpha}(\partial \Omega)\right) .
$$

The fact that $\left.E\right|_{S_{T}}=0$ implies $\left.\gamma \partial_{t} E\right|_{S_{T}}=0$. Recall from (4.42) that $\gamma \Delta F \in$ $L^{1}\left(0, T ; B^{-1 / \alpha, \alpha}(\partial \Omega)\right)$ so that from (6.18),

$$
\gamma \Delta E \in L^{1}\left(0, T ; B^{-1 / \alpha, \alpha}(\partial \Omega)\right), \quad 1<\alpha<2 .
$$

Repeating the arguments in the proof of Theorem 4.9 we obtain (6.13). To prove the trace result for $r$, we proceed in the same way as in the proof of Theorem 4.10 for $p$. Taking the divergence of (6.12) we obtain

$$
-\Delta r=G_{2}+\Delta p
$$

where

$$
G_{2}=\partial_{1} q_{2}\left(\partial_{1} w_{2}-\partial_{2} w_{1}\right)+\partial_{2} q_{1}\left(\partial_{2} w_{1}-\partial_{1} w_{2}\right)+q_{1} \Delta w_{1}+q_{2} \Delta w_{2} .
$$

Analogous to the proof of Lemma 4.5 we have

$$
G_{2} \in L^{1}\left(0, T ; W^{-1, \alpha}(\Theta)\right), \quad 1<\alpha<2 .
$$

Hence, the last three relations and the fact that $r \in L^{2}\left(0, T ; L^{2}(\Theta)\right)$ and $\Delta p \in$ $L^{1}\left(0, T ; W^{-1, \alpha}(\Theta)\right)$ yield

$$
r \in L^{1}\left(0, T ; X_{\alpha}\right)
$$

so that from Proposition 4.8, $\gamma r \in L^{1}\left(0, T ; B^{-1 / \alpha, \alpha}(\partial \Omega)\right)$ for $1<\alpha<2$; i.e., (6.14) holds. Finally, (6.15) follows trivially from (6.13)-(6.14).

We now establish some Green's formulae.

Lemma 6.4. Let $r \in X_{\alpha}(\Theta), 1<\alpha<2$. Then the distribution $\nabla r$ can be extended continuously into the functional defined by

$$
\langle\nabla r, \mathbf{h}\rangle=\langle r \mathbf{n}, \mathbf{h}\rangle_{\partial \Omega}-\int_{\Theta} r \operatorname{div} \mathbf{h} d \mathbf{x}
$$

for every $\mathbf{h} \in \mathbf{C}^{\infty}(\bar{\Theta})$ which vanishes near $(0, T) \times(\partial \Theta \backslash \partial \Omega)$. Here $\langle\nabla r, \cdot\rangle$ denotes the defined functional and $\langle\cdot, \cdot\rangle_{\partial \Omega}$ denotes the duality pairing between $\mathbf{B}^{1 / \alpha, \alpha^{\prime}}(\partial \Theta)$ and $\mathbf{B}^{-1 / \alpha, \alpha}(\partial \Theta)$.

Proof. By Lemma 4.7 we may choose a sequence $\left\{r_{n}\right\} \subset C^{\infty}(\bar{\Theta})$ such that $r_{n} \rightarrow r$ in $X_{\alpha}$. Formula (6.19) holds for $r=r_{n}$ by the classical Stokes theorem. Since the right side of (6.19) with $r=r_{n}$ converges as $n \rightarrow \infty$ to the same expression with $r$, formula (6.19) defines the desired functional for $r \in X_{\alpha}(\Theta)$.

REMARK. On $C^{\infty}(\bar{\Theta})$, the definition of the operator $\nabla$ found in Lemma 6.4 coincides with the classical definition.

LEMma 6.5. Let $\mathbf{w} \in Y$ be a solution of (3.1)-(3.3). Then there exists a sequence of solutions of (3.1), $\left\{\mathbf{w}^{(k)}\right\} \subset Y \cap L^{\infty}\left(0, T ; \mathbf{V}^{2}(\Theta)\right)$, such that $\mathbf{w}^{(k)} \rightarrow \mathbf{w}$ in $Y$.

Proof. Let $U \subset \Theta$ be a (bounded) neighborhood of $\partial \Omega$ such that the extension $\mathbf{u}$ of the boundary data $\mathbf{b}$ constructed in Theorem 4.2 has support in $U$ and we can choose a coordinate system $\left(x_{1}, x_{2}\right)^{T}$ such that $U=\left\{\left(x_{1}, x_{2}\right)^{T} \in \mathbb{R}^{2}: 0<x_{2}<d\right\}$ and $\partial \Omega=\left\{\left(x_{1}, x_{2}\right)^{T} \in \mathbb{R}^{2}: x_{2}=0\right\}$. We consider the sequence $\left\{\mathbf{u}^{(k)}\right\}$ defined by

$$
\mathbf{u}^{(k)}(t, \mathbf{x}) \equiv \int_{0}^{T} \int_{U} k^{3} \phi\left(k(t-s), k\left(x_{1}-y_{1}\right), k\left(x_{2}-y_{2}\right)\right) \mathbf{u}\left(s, y_{1}, y_{2}\right) d y_{1} d y_{2} d s
$$


where $\phi \in C_{0}^{\infty}\left(\mathbb{R}^{3}\right), \int_{\mathbb{R}^{3}} \phi d t d \mathbf{x}=1$, and $\operatorname{supp} \phi \in\left\{\left(t, x_{1}, x_{2}\right)^{T} \in \mathbb{R}^{3}:-1<t<\right.$ $\left.1,-1<x_{1}<1,-1<x_{2}<0\right\}$. Evidently, each $\mathbf{u}^{(k)} \in \mathbf{C}^{\infty}((0, T) \times \Theta)$ and

$$
\left\|\mathbf{u}-\mathbf{u}^{(k)}\right\|_{\mathcal{V}^{(1)}\left(Q_{T}\right)} \rightarrow 0 \quad \text { as } k \rightarrow \infty .
$$

We set

$$
\mathbf{b}^{(k)}=\left.\mathbf{u}^{(k)}\right|_{(0, T) \times \partial \Omega} .
$$

We choose a sequence $\left\{\mathbf{w}_{0}^{(k)}\right\} \subset \mathbf{V}^{2}(\Omega)$ such that

$$
\mathbf{w}_{0}^{(k)} \rightarrow \mathbf{w}_{0} \quad \text { in } \mathbf{V}^{0}(\Omega)
$$

and each $\mathbf{w}_{0}^{(k)}$ satisfies the compatibility condition

$$
\left.\left(\mathbf{w}_{0}^{(k)} \cdot \mathbf{n}\right)\right|_{\partial \Omega}=\left.\left(\mathbf{b}_{0}^{(k)} \cdot \mathbf{n}\right)\right|_{t=0} .
$$

Moreover, we choose $\mathbf{w}_{0}^{(k)}$ such that $\mathbf{w}_{0}^{(k)}-\left.\mathbf{u}^{(k)}\right|_{t=0} \in \mathbf{V}_{0}^{1}(\Omega)$. We then consider (3.1) with the boundary and initial conditions

$$
\left.\mathbf{w}\right|_{(0, T) \times \partial \Omega}=\mathbf{b}^{(k)} \quad \text { and }\left.\quad \mathbf{w}\right|_{t=0}=\mathbf{w}_{0}^{(k)} .
$$

Let $b_{n}^{(k)}$ and $b_{\tau}^{(k)}$ be the normal and tangential components of $\mathbf{b}^{(k)}$, respectively:

$$
\mathbf{b}^{(k)}=b_{n}^{(k)} \mathbf{n}+b_{\tau}^{(k)} \boldsymbol{\tau} .
$$

Clearly, $b_{n}^{(k)}, b_{\tau}^{(k)}$, and $\mathbf{w}_{0}^{(k)}$ satisfy conditions (4.12)-(4.14) and (4.18) of Theorem 4.4. Hence, by Theorem 4.4, there exists a solution $\mathbf{w}^{(k)} \in Y$ for (3.1) and (6.23). We now show that $\mathbf{w}^{(k)} \rightarrow \mathbf{w}$ in $Y$. We obviously have

$$
\left.\left(\mathbf{w}^{(k)}-\mathbf{w}\right)\right|_{(0, T) \times \partial \Omega}=\mathbf{b}^{(k)}-\mathbf{b} \rightarrow \mathbf{0}
$$

in $H^{1}\left(0, T ; \mathbf{L}^{2}(\partial \Omega)\right) \cap L^{2}\left(0, T ; \mathbf{H}^{1 / 2}(\partial \Omega)\right) \cap \mathbf{L}^{k}((0, T) \times \partial \Omega)$. Thus we only need to show $\mathbf{w}^{(k)} \rightarrow \mathbf{w}$ in $\mathcal{V}^{(1)}\left(Q_{T}\right)$. We rewrite $\mathbf{w}-\mathbf{w}^{(k)}$ in the form

$$
\mathbf{w}-\mathbf{w}^{(k)}=\left(\mathbf{u}-\mathbf{u}^{(k)}\right)+\boldsymbol{\eta}^{(k)},
$$

where $\boldsymbol{\eta}^{(k)}$ satisfies (4.20), (4.21) with $\boldsymbol{\eta}_{0}^{(k)}=\left(\mathbf{w}_{0}-\mathbf{w}_{0}^{(k)}\right)-\left.\left(\mathbf{u}-\mathbf{u}^{(k)}\right)\right|_{t=0}$, and the following analog of (4.19):

$$
\begin{aligned}
& \left\langle\partial_{t} \boldsymbol{\eta}^{(k)}(t), \mathbf{z}\right\rangle+\mu \int_{\Omega} \nabla \boldsymbol{\eta}^{(k)}(t): \nabla \mathbf{z} d \mathbf{x}+\int_{\Omega}\left(\boldsymbol{\eta}^{(k)}(t) \cdot \nabla\right) \mathbf{w}(t) \cdot \mathbf{z} d \mathbf{x} \\
& +\int_{\Omega}\left(\left(\mathbf{w}^{(k)}(t)+\mathbf{v}_{\infty}\right) \cdot \nabla\right) \boldsymbol{\eta}^{(k)}(t) \cdot \mathbf{z} d \mathbf{x}=\left\langle\mathbf{f}^{(k)}(t), \mathbf{z}\right\rangle \quad \forall \mathbf{z} \in \mathbf{V}^{1}(\Omega), \text { a.e. } t \in(0, T),
\end{aligned}
$$

where $\mathbf{f}^{(k)}$ is defined by the following analog of (4.22):

$$
\begin{aligned}
\left\langle\mathbf{f}^{(k)}(t), \mathbf{z}\right\rangle=- & \mu \int_{\Omega} \nabla\left(\mathbf{u}(t)-\mathbf{u}^{(k)}(t)\right): \nabla \mathbf{z} d \mathbf{x}-\left\langle\partial_{t}\left(\mathbf{u}(t)-\mathbf{u}^{(k)}(t)\right), \mathbf{z}\right\rangle \\
& -\int_{\Omega}\left(\left(\mathbf{u}(t)-\mathbf{u}^{(k)}(t)\right) \cdot \nabla\right) \mathbf{w}(t) \cdot \mathbf{z} d \mathbf{x} \\
& -\int_{\Omega}\left(\left(\mathbf{w}^{(k)}(t)+\mathbf{v}_{\infty}\right) \cdot \nabla\right)\left(\mathbf{u}(t)-\mathbf{u}^{(k)}(t)\right) \cdot \mathbf{z} d \mathbf{x} .
\end{aligned}
$$


We can estimate $\boldsymbol{\eta}^{(k)}$ and $\mathbf{f}^{(k)}$ as in the proof of Lemma 4.3 and (4.25) to obtain

$$
\begin{aligned}
& \left\|\boldsymbol{\eta}^{(k)}\right\|_{\mathcal{V}^{(1)}\left(Q_{T}\right)} \\
& \leq A\left(\left\|\mathbf{f}^{(k)}\right\|_{L^{2}\left(0, T ; \mathbf{V}^{-1}(\Omega)\right)},\left\|\mathbf{u}-\mathbf{u}^{(k)}\right\|_{\mathcal{V}^{(1)}\left(Q_{T}\right)},\left\|\boldsymbol{\eta}_{0}^{(k)}\right\|_{\mathbf{V}^{0}(\Omega)},\|\mathbf{w}\|_{\mathcal{V}^{(1)}\left(Q_{T}\right)},\left|\mathbf{v}_{\infty}\right|\right)
\end{aligned}
$$

and

$$
\begin{aligned}
\left\|\mathbf{f}^{(k)}\right\|_{L^{2}\left(0, T ; \mathbf{V}^{-1}(\Omega)\right)} \leq C\left(\left\|\mathbf{u}-\mathbf{u}^{(k)}\right\|_{\mathcal{V}^{(1)}\left(Q_{T}\right)}+\left\|\mathbf{u}-\mathbf{u}^{(k)}\right\|_{\mathcal{V}^{(1)}\left(Q_{T}\right)}\|\mathbf{w}\|_{\mathcal{V}^{(1)}\left(Q_{T}\right)}\right. \\
\left.+\left\|\mathbf{u}-\mathbf{u}^{(k)}\right\|_{\mathcal{V}^{(1)}\left(Q_{T}\right)}\left(\left\|\mathbf{w}^{(k)}\right\|_{\mathcal{V}^{(1)}\left(Q_{T}\right)}+\left|\mathbf{v}_{\infty}\right|\right)\right)
\end{aligned}
$$

where $A(\cdot)$ is a continuous positive function on $\mathbb{R}^{5}$ and $A\left(\lambda_{1}, \lambda_{2}, \lambda_{3}, \lambda_{4}, \lambda_{5}\right) \rightarrow 0$ as $\left(\lambda_{1}, \lambda_{2}, \lambda_{3}\right) \rightarrow(0,0,0)$ for fixed $\lambda_{4}, \lambda_{5}$. Taking into account $(6.21)-(6.22)$ and the boundedness of $\left\|\mathbf{w}^{(k)}\right\|_{\mathcal{V}^{(1)}\left(Q_{T}\right)}$ (which follows from Theorem 4.4), we obtain

$$
\left\|\boldsymbol{\eta}^{(k)}\right\|_{\mathcal{V}^{(1)}\left(Q_{T}\right)} \rightarrow 0 \quad \text { as } k \rightarrow \infty .
$$

Relations (6.21) and (6.25) imply the convergence $\mathbf{w}^{(k)} \rightarrow \mathbf{w}$ in $\mathcal{V}^{(1)}\left(Q_{T}\right)$. Finally, we prove that $\mathbf{w}^{(k)} \in L^{\infty}\left(0, T ; \mathbf{V}^{2}(\Theta)\right)$. To this end, we write $\mathbf{w}^{(k)}$ in the form

$$
\mathbf{w}^{(k)}=\mathbf{u}^{(k)}+\boldsymbol{\xi}^{(k)} .
$$

Then $\boldsymbol{\xi}^{(k)}$ is the solution of problem (4.19)-(4.21) with $\mathbf{u}$ replaced by $\mathbf{u}^{(k)}$ and $\mathbf{f}$ defined by (4.22), wherein $\mathbf{f}$ is replaced by $\mathbf{f}^{(k)}$ and $\mathbf{u}$ is replaced by $\mathbf{u}^{(k)}$. Note that by (6.20) the inclusion $\mathbf{u}^{(k)} \in \mathbf{C}^{\infty}(\bar{\Theta})$ holds. By (4.22) we have

$$
\mathbf{f}^{(k)}=P\left(\mu \Delta \mathbf{u}^{(k)}-\partial_{t} \mathbf{u}^{(k)}+\left[\left(\mathbf{u}^{(k)}+\mathbf{v}_{\infty}\right) \cdot \nabla\right] \mathbf{u}^{(k)}\right),
$$

where $P$ is the orthogonal projection from $\mathbf{L}^{2}(\Theta)$ onto $\mathbf{V}_{0}^{0}(\Theta)$. Since $\mathbf{u}^{(k)} \in \mathbf{C}^{\infty}(\bar{\Theta})$, we have $\mathbf{f}^{(k)} \in L^{\infty}\left(0, T ; \mathbf{V}_{0}^{0}(\Theta)\right), \partial_{t} \mathbf{f}^{(k)} \in L^{2}\left(0, T ; \widehat{\mathbf{V}}^{-1}(\Theta)\right)$, and $\left.\mathbf{f}^{(k)}\right|_{t=0} \in \mathbf{V}_{0}^{0}(\Theta)$, where $\widehat{\mathbf{V}}^{-1}$ is the completion of $\mathbf{V}_{0}^{0}$ under the norm $\sup _{\|\phi\|_{\mathbf{V}_{0}^{1}=1}} \int_{\Theta} f \cdot \phi d \mathbf{x}$. By (6.26) and by properties of $\left.\left(\mathbf{w}^{(k)}-\mathbf{u}^{(k)}\right)\right|_{t=0}$ we have that $\left.\boldsymbol{\xi}^{(k)}\right|_{t=0} \in \mathbf{V}^{2}(\Omega) \cap \mathbf{V}_{0}^{1}(\Omega)$. Hence, by a result in [28, pp. 299-302], we have $\boldsymbol{\xi}^{(k)} \in L^{\infty}\left(0, T ; \mathbf{V}^{2}(\Theta)\right)$ so that $\mathbf{w}^{(k)} \in$ $L^{\infty}\left(0, T ; \mathbf{V}^{2}(\Theta)\right)$.

We may prove a similar result for the solution $\mathbf{q}$ for the adjoint equation (6.12). We consider the boundary value problem

$(6.28)-\partial_{t} \mathbf{q}^{(k)}-\mu \Delta \mathbf{q}^{(k)}+\mathbf{q}^{(k)} \cdot\left(\nabla \mathbf{w}^{(k)}\right)^{T}-\left[\left(\mathbf{w}^{(k)}+\mathbf{v}_{\infty}\right) \cdot \nabla\right] \mathbf{q}^{(k)}+\nabla \widetilde{r}^{(k)}=\mu \Delta \mathbf{w}^{(k)}$,

$$
\operatorname{div} \mathbf{q}^{(k)}=0,
$$

and

$$
\left.\mathbf{q}^{(k)}\right|_{t=T}=\mathbf{q}_{0}^{(k)} \in \mathbf{V}_{0}^{0}(\Theta),\left.\quad \mathbf{q}^{(k)}\right|_{(0, T) \times \partial \Omega}=\mathbf{0} .
$$

The existence and uniqueness of the solution $\mathbf{q}^{(k)} \in \mathcal{V}^{(1)}\left(Q_{T}\right)$ for (6.28)-(6.30) can be shown by the standard techniques (see [19], [28]).

LEMma 6.6. Let $\mathbf{q} \in \mathcal{V}^{(1)}\left(Q_{T}\right) \cap L^{2}\left(0, T ; \mathbf{V}_{0}^{1}(\Omega)\right)$ and $\widetilde{r} \in L^{2}\left(0, T ; L_{\mathrm{loc}}^{2}(\Omega)\right)$ be the solution of (6.11), $\mathbf{w}^{(k)}$ be the solution of (3.1) and (6.22), and $\mathbf{q}^{(k)}$ be the solution 
of (6.28)-(6.30), where $\mathbf{q}_{0}^{(k)} \in \mathbf{V}_{0}^{1}(\Omega)$ and $\left\|\mathbf{q}_{0}^{(k)}-\mathbf{q}(T, \cdot)\right\|_{\mathbf{V}_{0}^{0}(\Omega)} \rightarrow 0$ as $k \rightarrow \infty$. Then for every $k, \mathbf{q}^{(k)} \in L^{2}\left(0, T ; \mathbf{V}^{2}(\Theta)\right)$ and $\left\|\mathbf{q}^{(k)}-\mathbf{q}\right\|_{\mathcal{V}^{(1)}\left(Q_{T}\right)} \rightarrow 0$ as $k \rightarrow \infty$.

Proof. By Lemma 6.5, $\left\|\mathbf{w}^{(k)}-\mathbf{w}\right\|_{\mathcal{V}^{(1)}\left(Q_{T}\right)} \rightarrow 0$ as $k \rightarrow \infty$. Subtracting (6.11) from (6.28) and doing estimation as in Lemma 4.3 we obtain

$$
\left\|\mathbf{q}^{(k)}-\mathbf{q}\right\|_{\mathcal{V}^{(1)}\left(Q_{T}\right)} \rightarrow 0 \quad \text { as } k \rightarrow \infty .
$$

By Lemma $6.5, \mathbf{w}^{(k)} \in \mathcal{V}^{(1)}\left(Q_{T}\right) \cap L^{\infty}\left(0, T ; \mathbf{V}^{2}(\Theta)\right)$. Also, $\mathbf{q}^{(k)} \in \mathcal{V}^{(1)}\left(Q_{T}\right)$. Thus we have that

$$
\mathbf{g} \equiv \mu \Delta \mathbf{w}^{(k)}-\mathbf{q}^{(k)} \cdot\left(\nabla \mathbf{w}^{(k)}\right)^{T}-\left[\left(\mathbf{w}^{(k)}+\mathbf{v}_{\infty}\right) \cdot \nabla\right] \mathbf{q}^{(k)} \in \mathbf{L}^{2}((0, T) \times \Theta) .
$$

We rewrite (6.28) in the form

$$
-\partial_{t} \mathbf{q}^{(k)}-\mu \Delta \mathbf{q}^{(k)}+\nabla \widetilde{r}^{(k)}=\mathbf{g} .
$$

Applying to this last equation the regularity result for the Stokes equations (see [19]) we obtain $\mathbf{q}^{(k)} \in L^{2}\left(0, T ; \mathbf{V}^{2}(\Theta)\right)$.

LEMMA 6.7. Let $\mathbf{w} \in Y$ be a solution of problem (3.1)-(3.3) and $\mathbf{q} \in \mathcal{V}^{(1)}\left(Q_{T}\right) \cap$ $L^{2}\left(0, T ; \mathbf{V}_{0}^{1}(\Omega)\right)$ be a solution of the adjoint equation (6.11). Then the distribution $\Delta \mathbf{w}$ defined on $\mathbf{C}_{0}^{\infty}(\Omega) \cap \mathbf{V}^{0}(\Omega)$ can be extended continuously into a functional defined by

$$
\int_{\Theta} \Delta \mathbf{w} \cdot \mathbf{h} d \mathbf{x}=\int_{\partial \Omega}\left((\nabla \mathbf{w})+(\nabla \mathbf{w})^{T}\right) \mathbf{n} \cdot \mathbf{h} d s-2 \int_{\Theta} \mathcal{D}(\mathbf{w}): \mathcal{D}(\mathbf{h}) d \mathbf{x}
$$

for every $\mathbf{h} \in \mathbf{C}^{\infty}(\bar{\Theta})$ which vanishes near $(0, T) \times(\partial \Theta \backslash \partial \Omega)$. Furthermore, the time-dependent version of (6.31) also holds; i.e.,

$$
\begin{aligned}
\int_{0}^{T} & \int_{\Theta} \Delta \mathbf{w} \cdot \mathbf{h} d \mathbf{x} d t \\
\quad & \int_{0}^{T} \int_{\partial \Omega}\left((\nabla \mathbf{w})+(\nabla \mathbf{w})^{T}\right) \mathbf{n} \cdot \mathbf{h} d s d t-2 \int_{0}^{T} \int_{\Theta} \mathcal{D}(\mathbf{w}): \mathcal{D}(\mathbf{h}) d \mathbf{x} d t
\end{aligned}
$$

for every $\mathbf{h} \in \mathbf{C}^{\infty}((0, T) \times \bar{\Theta})$ which vanishes near $(0, T) \times(\partial \Theta \backslash \partial \Omega)$. Formulae (6.31) and (6.32) also hold if $\mathbf{w}$ is replaced by $\mathbf{q}$.

Proof. If $\mathbf{w}=\mathbf{w}^{(k)} \in L^{\infty}\left(0, T ; \mathbf{V}^{2}(\Theta)\right)$, (6.32) is the well-known Green's formula (see (2.5) and the ensuing formulae). We substitute into (6.32) the solution $\mathbf{w}^{(k)}$ for the problem (3.1) and (6.23) as constructed in Lemma 6.5. By this lemma we have $\mathbf{w}^{(k)} \rightarrow \mathbf{w}$ in $\mathcal{V}^{(1)}\left(Q_{T}\right)$, and therefore,

$$
\int_{0}^{T} \int_{\Theta} \mathcal{D}\left(\mathbf{w}^{(k)}\right): \mathcal{D}(\mathbf{h}) d \mathbf{x} d t \rightarrow \int_{0}^{T} \int_{\Theta} \mathcal{D}(\mathbf{w}): \mathcal{D}(\mathbf{h}) d \mathbf{x} d t
$$

as $k \rightarrow \infty$. Theorem 4.9 yields that the operator $\mathbf{w} \mapsto \gamma\left((\nabla \mathbf{w})+(\nabla \mathbf{w})^{T}\right) \mathbf{n}$ is continuous from the set $\{\mathbf{w} \in Y: \mathbf{w}$ satisfies $(3.1)\}$ to the space $L^{1}\left(0, T ; \mathbf{B}^{-1 / \alpha, \alpha}(\partial \Omega)\right)$, $1<\alpha<2$. Hence,

$$
\int_{0}^{T} \int_{\partial \Omega}\left(\left(\nabla \mathbf{w}^{(k)}\right)+\left(\nabla \mathbf{w}^{(k)}\right)^{T}\right) \mathbf{n} \cdot \mathbf{h} d s d t \rightarrow \int_{0}^{T} \int_{\partial \Omega}\left((\nabla \mathbf{w})+(\nabla \mathbf{w})^{T}\right) \mathbf{n} \cdot \mathbf{h} d s d t
$$

as $k \rightarrow \infty$. Substituting $\mathbf{w}=\mathbf{w}^{(k)}$ into (6.32) and passing to the limit as $k \rightarrow \infty$ in the right-hand side of this formula we obtain the desired extension of the distribution $\Delta \mathbf{w}$ which is defined by (6.32). The steady state formula (6.31) can be similarly proved. The case of the distribution $\Delta \mathbf{q}$ can be proved analogously. 
6.4. The optimality system in the form of a boundary value problem for a system of partial differential equations. We now interpret the optimality system (3.1), (3.3), and (6.4) as a system of partial differential equations with boundary conditions on the entire boundary of the cylinder $Q_{T}=(0, T) \times \Omega$.

We first recall from section 3 that

$$
\mathbf{V}^{0}(\Omega)=\left\{\mathbf{v} \in \mathbf{L}^{2}(\Omega): \operatorname{div} \mathbf{v}=0\right\}
$$

and

$$
\mathbf{V}_{0}^{0}(\Omega)=\text { the closure of } \mathbf{C}_{0}^{\infty}(\Omega) \cap \mathbf{V}^{0}(\Omega) \text { in the } \mathbf{L}^{2}(\Omega) \text {-norm . }
$$

We have the well-known Weyl decomposition (see [14] and [19])

$$
\mathbf{L}^{2}(\Omega)=\mathbf{V}_{0}^{0}(\Omega) \oplus\left(\nabla H^{1}(\Omega)\right),
$$

where $\nabla H^{1}(\Omega)=\left\{\nabla g: g \in H^{1}(\Omega)\right\}$. We claim that

$$
\mathbf{V}^{0}(\Omega)=\mathbf{V}_{0}^{0}(\Omega) \oplus\left(\nabla H_{\pi}\right),
$$

where $\nabla H_{\pi}=\left\{\nabla g: g \in H^{1}(\Omega), \Delta g=0\right\}$. Indeed, since for each $\mathbf{w} \in \mathbf{V}^{0}(\Omega)$ we have $\mathbf{w}=\mathbf{w}_{\sigma}+\nabla w_{\pi}$ from the Weyl decomposition with $\mathbf{w}_{\sigma} \in \mathbf{V}_{0}^{0}(\Omega)$ and $w_{\pi} \in H^{1}(\Omega)$, we obtain by taking the divergence of $\mathbf{w}$ that $\Delta w_{\pi}=\operatorname{div} \mathbf{w}-\operatorname{div} \mathbf{w}_{\sigma}=0$.

We are now prepared to interpret the optimality system in the weak form as a system of partial differential equations with boundary conditions on the entire boundary of the cylinder $Q_{T}=(0, T) \times \Omega$.

THEOREM 6.8. Assume $\mathbf{w} \in Y$ is a solution for Problem I and $\mathbf{q} \in \mathcal{V}^{(1)}\left(Q_{T}\right)$ is as defined in Theorem 6.2. Then there exist a $p \in L^{2}\left(0, T ; L_{\mathrm{loc}}^{2}(\Omega)\right)$ and an $r \in$ $L^{2}\left(0, T ; L_{\mathrm{loc}}^{2}(\Omega)\right)$ such that the quadruplet $(\mathbf{w}, p, \mathbf{q}, r)$ satisfies the partial differential equations (in the sense of distributions)

$$
\begin{gathered}
\partial_{t} \mathbf{w}-\mu \Delta \mathbf{w}+\left(\left(\mathbf{w}+\mathbf{v}_{\infty}\right) \cdot \nabla\right) \mathbf{w}+\nabla p=\mathbf{0} \quad \text { in }(0, T) \times \Omega, \\
\nabla \cdot \mathbf{w}=0 \quad \text { in }(0, T) \times \Omega, \\
-\partial_{t} \mathbf{q}-\mu \Delta \mathbf{q}+\mathbf{q} \cdot(\nabla \mathbf{w})^{T}-(\mathbf{w} \cdot \nabla) \mathbf{q}-\left(\mathbf{v}_{\infty} \cdot \nabla\right) \mathbf{q}+\nabla r=\mu \Delta \mathbf{w}-\nabla p,
\end{gathered}
$$

and

$$
\nabla \cdot \mathbf{q}=0 \quad \text { in }(0, T) \times \Omega,
$$

the initial and terminal conditions

$$
\mathbf{w}(0, \cdot)=\mathbf{w}_{0}(\cdot) \quad \text { in } \mathbf{V}^{0}(\Omega)
$$

and

$$
\mathbf{q}(T, \cdot)+\frac{1}{2} \mathbf{w}_{\sigma}(T, \cdot)=0 \quad \text { in } \mathbf{V}_{0}^{0}(\Omega),
$$

and the (lateral) boundary condition

$$
\left.\mathbf{q}\right|_{S_{T}}=\mathbf{0},
$$


where $\mathbf{w}_{\sigma}(T, \cdot)$ is the projection of $\mathbf{w}(T, \cdot)$ onto $\mathbf{V}_{0}^{0}(\Omega)$. Moreover,

$$
\begin{gathered}
\partial_{t t}(\gamma \mathbf{w}) \in L^{1}\left(0, T ; \mathbf{B}^{-1 / \alpha, \alpha}(\partial \Omega)\right), \\
\gamma\left[\left((\nabla \mathbf{w})+(\nabla \mathbf{w})^{T}\right) \cdot \mathbf{n}\right] \in L^{1}\left(0, T ; \mathbf{B}^{-1 / \alpha, \alpha}(\partial \Omega)\right), \\
\gamma p \in L^{1}\left(0, T ; B^{-1 / \alpha, \alpha}(\partial \Omega)\right), \quad \int_{\partial \Omega} p d s=0, \\
\gamma\left[\left((\nabla \mathbf{q})+(\nabla \mathbf{q})^{T}\right) \cdot \mathbf{n}\right] \in L^{1}\left(0, T ; \mathbf{B}^{-1 / \alpha, \alpha}(\partial \Omega)\right),
\end{gathered}
$$

and

$$
\gamma r \in L^{1}\left(0, T ; B^{-1 / \alpha, \alpha}(\partial \Omega)\right), \quad \int_{\partial \Omega} r d s=0
$$

where $1<\alpha<2$ and the following boundary conditions hold:

$$
2 N \partial_{t t}^{2}(\gamma \mathbf{w})-\mathcal{A}(\mathbf{w})-\mathcal{T}(\mathbf{w}, p) \mathbf{n}-\mathcal{T}(\mathbf{q}, r) \mathbf{n}=\eta(t) \mathbf{n}
$$

where

$$
\begin{gathered}
\mathcal{T}(\mathbf{w}, p)=-p I+2 \mu \mathcal{D}(\mathbf{w}) \quad \text { and } \quad \mathcal{T}(\mathbf{q}, r)=-r I+2 \mu \mathcal{D}(\mathbf{q}) \\
\mathcal{A}(\mathbf{w})=\gamma\left(k N\left|\mathbf{w}+\mathbf{v}_{\infty}\right|^{k-2}\left(\mathbf{w}+\mathbf{v}_{\infty}\right)+\frac{1}{2}\left(\left(\mathbf{w}+\mathbf{v}_{\infty}\right) \cdot \mathbf{n}\right) \mathbf{w}+\frac{|\mathbf{w}|^{2}}{4} \mathbf{n}\right)
\end{gathered}
$$

and

$$
\eta(t)=-\int_{\partial \Omega} \mathcal{A}(\mathbf{w}) \cdot \mathbf{n} d s / \int_{\partial \Omega} d s .
$$

Furthermore, the following compatibility conditions hold:

$$
\begin{gathered}
{\left.[(\gamma \mathbf{w}) \cdot \mathbf{n}]\right|_{t=0}=\left(\gamma \mathbf{w}_{0}\right) \cdot \mathbf{n},} \\
\left.\left(\partial_{t} \gamma \mathbf{w}\right) \cdot \boldsymbol{\tau}\right|_{t=T}=0 \quad \text { and }\left.\quad\left(\frac{1}{2} \gamma w_{\pi}+2 N \partial_{t} \gamma \mathbf{w} \cdot \mathbf{n}\right)\right|_{t=T}=0,
\end{gathered}
$$

where $\boldsymbol{\tau}$ is the unit tangential along $\partial \Omega$ and $w_{\pi}(t, \cdot)$ is the primitive function of the projection of $\mathbf{w}(t, \cdot)$ onto $\nabla H_{\pi}$ determined by the condition

$$
\int_{\partial \Omega} w_{\pi}(t, \cdot) d s=0
$$

Proof. $\mathbf{w} \in \mathcal{V}^{(1)}\left(Q_{T}\right)$ as a solution of Problem I satisfies (3.1) and (6.37). By the De Rham lemma, or recall from (4.27), there exists a $p \in L^{2}\left(0, T ; L_{\text {loc }}^{2}(\Omega)\right)$ such that (6.33) holds. Relation (6.49) follows from the inclusion $\mathbf{w} \in Y$ and the remark at the end of section 4.1. For the Lagrange multiplier $\mathbf{q} \in \mathcal{V}^{(1)}\left(Q_{T}\right) \cap L^{2}\left(0, T ; \mathbf{V}_{0}^{1}\right)$ we recall from (6.12) that there exists an $r \in L^{2}\left(0, T ; L_{\text {loc }}^{2}(\Omega)\right)$ such that (6.35) holds. (6.34) and (6.36) simply follow from the fact that $\mathbf{w} \in \mathcal{V}^{(1)}\left(Q_{T}\right)$ and $\mathbf{q} \in \mathcal{V}^{(1)}\left(Q_{T}\right) ;(6.39)$ 
follows from the fact that $\mathbf{q} \in L^{2}\left(0, T ; \mathbf{V}_{0}^{1}(\Omega)\right)$. From Theorem 4.10 and Lemma 6.3 we see that the traces of $p$ and $r$ live in the space $L^{1}\left(0, T ; B^{-1 / \alpha, \alpha}(\partial \Omega)\right)$ for $1<\alpha<2$. Also, note that in (6.33) and (6.35), we can add an arbitrary constant to $p$ and $r$ so that, in particular, we can choose $p$ and $r$ satisfying $\int_{\partial \Omega} p d s=0$ and $\int_{\partial \Omega} r d s=0$, respectively, where the integrals are understood as the duality pairings $\langle p, 1\rangle$ and $\langle r, 1\rangle$, respectively. This eliminates the arbitrary constant from $p$ and $r$ and also will facilitate some later discussion. Hence (6.42) and (6.44) are verified. Relations (6.41)-(6.44) follow from Theorems 4.9 and 4.10 and Lemma 6.3.

We now examine (6.45). By taking $\mathbf{h} \in \mathbf{C}^{\infty}$ in (6.4) with $\operatorname{div} \mathbf{h}=0,\left.\mathbf{h}\right|_{t=0}=\mathbf{0}$, $\left.\mathbf{h}\right|_{t=T}=\mathbf{0}$ and integrating by parts (which is justified by Lemma 6.7), we obtain

$$
\begin{aligned}
& \int_{0}^{T} \int_{\Omega}\left(-\partial_{t} \mathbf{q}-\mu \Delta \mathbf{q}+\mathbf{q} \cdot(\nabla \mathbf{w})^{T}-\left[\left(\mathbf{w}+\mathbf{v}_{\infty}\right) \cdot \nabla\right] \mathbf{q}-\mu \Delta \mathbf{w}\right) \cdot \mathbf{h} d \mathbf{x} d t \\
& \quad+\int_{0}^{T} \int_{\partial \Omega}\left(2 N \partial_{t} \mathbf{w} \cdot \partial_{t} \mathbf{h}+(\mathcal{A}(\mathbf{w})+2 \mu \mathcal{D}(\mathbf{w}) \mathbf{n}+2 \mu \mathcal{D}(\mathbf{q}) \mathbf{n}) \cdot \mathbf{h}\right) d s d t=0
\end{aligned}
$$

where $\mathcal{A}(\mathbf{w})$ is defined by (6.47). Also, the integrals are understood as duality pairings if necessary. Equations (6.35) and (6.52) yield

$$
\begin{aligned}
- & \int_{0}^{T} \int_{\Omega}(\nabla r+\nabla p) \cdot \mathbf{h} d \mathbf{x} d t \\
& +\int_{0}^{T} \int_{\partial \Omega}\left(2 N \partial_{t} \mathbf{w} \cdot \partial_{t} \mathbf{h}+(\mathcal{A}(\mathbf{w})+2 \mu \mathcal{D}(\mathbf{w}) \mathbf{n}+2 \mu \mathcal{D}(\mathbf{q}) \mathbf{n}) \cdot \mathbf{h}\right) d s d t=0 .
\end{aligned}
$$

Using Lemma 6.4 and the last equation, we obtain

$$
\int_{0}^{T} \int_{\partial \Omega}\left(2 N \partial_{t} \mathbf{w} \cdot \partial_{t} \mathbf{h}+(\mathcal{A}(\mathbf{w})+\mathcal{T}(p, \mathbf{w}) \mathbf{n}+\mathcal{T}(r, \mathbf{q}) \mathbf{n}) \cdot \mathbf{h}\right) d s d t=0,
$$

where $\mathcal{T}$ is the stress tensor defined by (6.46). Since $1<\alpha<2$, we have the continuous imbeddings $B^{1 / \alpha, \alpha^{\prime}}(\partial \Omega) \hookrightarrow L^{\infty}(\partial \Omega)$, where $\alpha^{\prime}=\alpha /(\alpha-1)$ so that $L^{1}(\partial \Omega) \hookrightarrow B^{-1 / \alpha, \alpha}(\partial \Omega)$. Hence,

$\gamma\left(2 k N\left|\mathbf{w}+\mathbf{v}_{\infty}\right|^{k-2}\left(\mathbf{w}+\mathbf{v}_{\infty}\right)+\frac{1}{2}\left(\left(\mathbf{w}+\mathbf{v}_{\infty}\right) \cdot \mathbf{n}\right) \mathbf{w}+\frac{|\mathbf{w}|^{2}}{4} \mathbf{n}\right) \in \mathbf{L}^{1}(\partial \Omega) \hookrightarrow \mathbf{B}^{-1 / \alpha, \alpha}(\partial \Omega)$.

Using Theorems 4.9 and 4.10, Lemma 6.3, and the last relation, we see that

$$
\mathcal{A}(\mathbf{w})+\mathcal{T}(p, \mathbf{w}) \mathbf{n}+\mathcal{T}(r, \mathbf{q}) \mathbf{n} \in L^{1}\left(0, T ; \mathbf{B}^{-1 / \alpha, \alpha}(\partial \Omega)\right) .
$$

Since $\mathbf{h}$ and $\partial_{t} \mathbf{w}$ are solenoidal (from the definition of the spaces $\mathcal{V}^{(1)}\left(Q_{T}\right)$ and $Y$ ), we have

$$
\int_{\partial \Omega} \mathbf{h} \cdot \mathbf{n} d s=0 \quad \text { and } \quad \int_{\partial \Omega} \partial_{t} \mathbf{w} \cdot \mathbf{n} d s=0 .
$$

Thus, from (6.54)-(6.55), we deduce (6.40) and

$$
\int_{0}^{T} \int_{\partial \Omega}\left(2 N \partial_{t t} \mathbf{w}-\mathcal{A}(\mathbf{w})-\mathcal{T}(p, \mathbf{w}) \mathbf{n}-\mathcal{T}(r, \mathbf{q}) \mathbf{n}\right) \cdot \mathbf{h} d s d t=0 .
$$

Hence, (6.45) follows from (6.55) and (6.56) with $\eta(t)$ defined by

$$
\eta(t)=\int_{\partial \Omega} \mathbf{n} \cdot\left(2 N \partial_{t t} \mathbf{w}-\mathcal{A}(\mathbf{w})-\mathcal{T}(p, \mathbf{w}) \mathbf{n}-\mathcal{T}(r, \mathbf{q}) \mathbf{n}\right) d s / \int_{\partial \Omega} d s .
$$


Note that for every $\phi(t) \in C_{0}^{\infty}(0, T)$,

$$
\int_{0}^{T} \int_{\partial \Omega} \partial_{t t} \mathbf{w} \cdot \mathbf{n} d s \phi(t) d t=\int_{0}^{T} \int_{\partial \Omega} \mathbf{w} \cdot \mathbf{n} d s \partial_{t t} \phi(t) d t=0
$$

as $\int_{\partial \Omega} \mathbf{w} \cdot \mathbf{n} d s=0$ for every divergence-free function $\mathbf{w}$. Thus, for almost all $t \in$ $(0, T)$,

$$
\int_{\partial \Omega} \partial_{t t} \mathbf{w} \cdot \mathbf{n} d s=0
$$

Taking into account (6.42) we obtain for each $\phi(t) \in C_{0}^{\infty}(0, T)$,

$$
\begin{aligned}
& \int_{0}^{T} \int_{\partial \Omega} \mathbf{n} \cdot \mathcal{T}(p, \mathbf{w}) \mathbf{n} d s \phi(t) d t \\
& =\int_{0}^{T} \int_{\partial \Omega}\left(\mathbf{n} \cdot \mu\left((\nabla \mathbf{w})+(\nabla \mathbf{w})^{T}\right) \mathbf{n}-p\right) d s \phi(t) d t \\
& =\int_{0}^{T} \int_{\partial \Omega}\left(\mathbf{n} \cdot \mu\left((\nabla \mathbf{w})+(\nabla \mathbf{w})^{T}\right) \mathbf{n}\right) d s \phi(t) d t
\end{aligned}
$$

Let $\epsilon>0$ be given. For each $s \in \partial \Omega$, we consider the normal $\widetilde{\mathbf{n}}(s)$ to $\partial \Omega$ which is directed into $\Omega$. We choose the point $K(\epsilon, s)$ along $\widetilde{\mathbf{n}}(s)$ such that the distance between $K(\epsilon, s)$ and $\partial \Omega$ equals $\epsilon$. If $\epsilon$ is sufficiently small, then the set $\{K(\epsilon, s): s \in \partial \Omega\}$ is a $C^{\infty}$-manifold which we denote by $\partial \Omega_{\epsilon}$. Since $\mathbf{w} \in \mathbf{V}^{0}(\Omega)$,

$$
\int_{\partial \Omega} \mathbf{w} \cdot \widetilde{\mathbf{n}} d s=0 \quad \text { and } \quad \int_{\partial \Omega_{\epsilon}} \mathbf{w} \cdot \widetilde{\mathbf{n}}_{\epsilon} d s=0
$$

where $\widetilde{\mathbf{n}}_{\epsilon}$ is the outward normal to $\partial \Omega_{\epsilon}$. Hence,

$$
\begin{aligned}
& \int_{0}^{T} \int_{\partial \Omega}\left(\mathbf{n} \cdot\left((\nabla \mathbf{w})+(\nabla \mathbf{w})^{T}\right) \mathbf{n}\right) d s \phi(t) d t \\
& \quad=2 \int_{0}^{T} \int_{\partial \Omega} \partial_{n} \mathbf{w} \cdot \mathbf{n} d s \phi(t) d t \\
& \quad=\lim _{\epsilon \rightarrow 0} \frac{1}{\epsilon} \int_{0}^{T}\left(\int_{\partial \Omega} \mathbf{w} \cdot \mathbf{n} d s-\int_{\partial \Omega_{\epsilon}} \mathbf{w} \cdot \widetilde{\mathbf{n}}_{\epsilon} d s\right) \phi(t) d t=0 .
\end{aligned}
$$

Thus, (6.59) and (6.60) yield

$$
\int_{\partial \Omega} \mathbf{n} \cdot \mathcal{T}(p, \mathbf{w}) \mathbf{n} d s=0 \quad \text { for almost every } t \in(0, T) .
$$

Similarly, we have

$$
\int_{\partial \Omega} \mathbf{n} \cdot \mathcal{T}(r, \mathbf{q}) \mathbf{n} d s=0 \quad \text { for almost every } t \in(0, T) .
$$

From relations (6.58) and (6.61)-(6.62) we conclude that the function $\eta(t)$ defined in (6.57) equals the function defined in (6.48). 
Now we choose $\mathbf{h} \in \mathbf{C}^{\infty}\left(Q_{T}\right)$ in (6.4) with $\operatorname{div} \mathbf{h}=0$ and $\left.\mathbf{h}\right|_{t=0}=\mathbf{0}$. Integration by parts (which again is justified by Lemmas 6.4 and 6.7 ) yields

$$
\begin{aligned}
& \int_{0}^{T} \int_{\Omega}\left(-\partial_{t} \mathbf{q}-\mu \Delta \mathbf{q}+\mathbf{q} \cdot(\nabla \mathbf{w})^{T}-\left[\left(\mathbf{w}+\mathbf{v}_{\infty}\right) \cdot\right] \mathbf{q}-\mu \Delta \mathbf{w}\right) \cdot \mathbf{h} d \mathbf{x} d t \\
& +\int_{0}^{T} \int_{\partial \Omega}\left(-2 N \partial_{t t} \mathbf{w} \cdot \mathbf{h}+(\mathcal{A}(\mathbf{w})+2 \mu \mathcal{D}(\mathbf{w}) \mathbf{n}+2 \mu \mathcal{D}(\mathbf{q}) \mathbf{n}) \cdot \mathbf{h}\right) d s d t \\
& +\int_{\Omega}\left[\mathbf{q}(T, \mathbf{x})+\frac{1}{2} \mathbf{w}(T, \mathbf{x})\right] \cdot \mathbf{h}(T, \mathbf{x}) d \mathbf{x}+\int_{\partial \Omega} 2 N \partial_{t} \mathbf{w}(T, \mathbf{x}) \cdot \mathbf{h}(T, \mathbf{x}) d s=0 .
\end{aligned}
$$

Note that (6.53) and (6.56) hold for the present $\mathbf{h}$ so that using (6.35), (6.53), (6.56), and (6.63), we are led to

$$
\int_{\Omega}\left[\mathbf{q}(T, \mathbf{x})+\frac{1}{2} \mathbf{w}(T, \mathbf{x})\right] \cdot \mathbf{h}(T, \mathbf{x}) d \mathbf{x}+\int_{\partial \Omega} 2 N \partial_{t} \mathbf{w}(T, \mathbf{x}) \cdot \mathbf{h}(T, \mathbf{x}) d s=0 .
$$

Using the fact that $\mathbf{q}(T, \cdot) \in \mathbf{V}_{0}^{0}(\Omega)$ and $\mathbf{w}(T, \cdot) \in \mathbf{V}^{0}(\Omega)$ we obtain (6.38). Substituting (6.38) into (6.64) we obtain by integration by parts that

$$
\int_{\partial \Omega}\left(\frac{1}{2} w_{\pi}(T, \mathbf{x}) \mathbf{n}(\mathbf{x}) \cdot \mathbf{h}(T, \mathbf{x})+2 N \partial_{t} \mathbf{w}(T, \mathbf{x}) \cdot \mathbf{h}(T, \mathbf{x})\right) d s=0,
$$

which implies (6.50) with (6.51).

6.5. The case of Problem II. We derive now the optimality system for Problem II.

THEOREM 6.9. Assume that $\mathbf{v}_{0} \equiv \mathbf{w}_{0}+\mathbf{v}_{\infty} \in \mathbf{V}_{0}^{1}(\Omega)$ and $\mathbf{w} \in Y$ is a solution of Problem II. Then there exists a triplet $(\mathbf{q}, r, \lambda) \in \mathcal{V}^{(1)}\left(Q_{T}\right) \times L_{\mathrm{loc}}^{2}\left(Q_{T}\right) \times \mathbb{R}_{+}$and $p \in L_{\mathrm{loc}}^{2}\left(Q_{T}\right)$ such that $(\mathbf{q}, r, \lambda) \neq(\mathbf{0}, 0,0)$ and the collection $(\mathbf{w}, \mathbf{q}, p, r, \lambda)$ satisfies (6.33)-(6.44) and the boundary conditions

$$
2 \lambda \partial_{t t}^{2} \gamma \mathbf{w}-\widetilde{\mathcal{A}}(\mathbf{w})-\mathcal{T}(\mathbf{w}, p) \mathbf{n}-\mathcal{T}(\mathbf{q}, r) \mathbf{n}=\widetilde{\eta}(t) \mathbf{n},
$$

where $\mathcal{T}(\mathbf{w}, p)$ and $\mathcal{T}(\mathbf{q}, r)$ are defined by $(6.46)$,

$$
\widetilde{\mathcal{A}}(\mathbf{w})=\gamma\left(k \lambda\left|\mathbf{w}+\mathbf{v}_{\infty}\right|^{k-2}\left(\mathbf{w}+\mathbf{v}_{\infty}\right)+\frac{1}{2}\left(\left(\mathbf{w}+\mathbf{v}_{\infty}\right) \cdot \mathbf{n}\right) \mathbf{w}+\frac{|\mathbf{w}|^{2}}{4} \mathbf{n}\right),
$$

and

$$
\widetilde{\eta}(t)=-\int_{\partial \Omega} \widetilde{\mathcal{A}}(\mathbf{w}) \cdot \mathbf{n} d s / \int_{\partial \Omega} d s .
$$

Moreover, the following compatibility conditions hold:

$$
\begin{gathered}
\left.(\gamma \mathbf{w}) \cdot \mathbf{n}\right|_{t=0}=\left(\gamma \mathbf{w}_{0}\right) \cdot \mathbf{n}, \\
\left.\lambda\left[\left(\partial_{t} \gamma \mathbf{w}\right) \cdot \boldsymbol{\tau}\right]\right|_{t=T}=0, \quad \text { and }\left.\quad\left(\frac{1}{2} \gamma w_{\pi}+2 \lambda \partial_{t} \gamma \mathbf{w} \cdot \mathbf{n}\right)\right|_{t=T}=0,
\end{gathered}
$$

where $\boldsymbol{\tau}$ is the unit tangential along $\partial \Omega$ and $w_{\pi}(t, \cdot)$ is the primitive function of the projection of $\mathbf{w}(t, \cdot)$ onto $\nabla H_{\pi}$ determined by the condition (6.51). Furthermore, the conditions of nonnegativeness and complementary slackness are valid; i.e.,

$$
\lambda \geq 0
$$


and

$$
\lambda\left(\int_{0}^{T} \int_{\partial \Omega}\left(\left|\mathbf{w}+\mathbf{v}_{\infty}\right|^{k}+\left|\partial_{t} \mathbf{w}\right|^{2}\right) d s d t-M\right)=0 .
$$

Proof. Let $\mathbf{w} \in Y$ be a solution of Problem II. We fit Problem II into the framework of Theorem 6.1. We set $X_{1}=Y_{0}$ and $X_{2}=L^{2}\left(0, T ; \mathbf{V}^{-1}(\Omega)\right)$. The Lagrange functional for Problem II is defined by

$$
\begin{aligned}
\mathcal{L}(\mathbf{y}, \mathbf{q})= & \lambda_{0}\left(\mu \int_{0}^{T} \int_{\Omega}|\mathcal{D}(\mathbf{w}+\mathbf{y})|^{2} d \mathbf{x} d t+\frac{1}{4} \int_{\Omega}|\mathbf{w}(T, \mathbf{x})+\mathbf{y}(T, \mathbf{x})|^{2} d \mathbf{x}\right. \\
& \left.+\frac{1}{4} \int_{0}^{T} \int_{\partial \Omega}|\mathbf{w}+\mathbf{y}|^{2}\left(\mathbf{w}+\mathbf{y}+\mathbf{v}_{\infty}\right) \cdot \mathbf{n} d s d t\right) \\
& +\lambda \int_{0}^{T} \int_{\partial \Omega}\left(\left|\mathbf{w}+\mathbf{y}+\mathbf{v}_{\infty}\right|^{k}+\left|\partial_{t} \mathbf{w}+\partial_{t} \mathbf{y}\right|^{2}-M\right) d s d t \\
& +\int_{0}^{T} \int_{\Omega}\left(\partial_{t} \mathbf{w}+\partial_{t} \mathbf{y}\right) \cdot \mathbf{q} d \mathbf{x} d t+2 \mu \int_{0}^{T} \int_{\Omega} \mathcal{D}(\mathbf{w}+\mathbf{y}): \mathcal{D}(\mathbf{q}) d \mathbf{x} d t \\
& +\int_{0}^{T} \int_{\Omega}\left\{[(\mathbf{w}+\mathbf{y}) \cdot \nabla](\mathbf{w}+\mathbf{y})+\left(\mathbf{v}_{\infty} \cdot \nabla\right)(\mathbf{w}+\mathbf{y})\right\} \cdot \mathbf{q} d \mathbf{x} d t
\end{aligned}
$$

for all $\mathbf{y} \in X_{1}$ and $\mathbf{q} \in X_{2}^{*}=L^{2}\left(0, T ; \mathbf{V}_{0}^{1}(\Omega)\right)$. Note that (6.72) differs from (6.9) in that $\lambda_{0}$ has to be included in the Lagrangian functional and $\lambda_{0}$ can be zero. We define the functionals

$$
f(\mathbf{y})=\mathcal{J}(\mathbf{w}+\mathbf{y})
$$

and

$$
g_{1}(\mathbf{y})=\int_{0}^{T} \int_{\partial \Omega}\left(\left|\mathbf{w}+\mathbf{y}+\mathbf{v}_{\infty}\right|^{k}+\left|\partial_{t} \mathbf{w}+\partial_{t} \mathbf{y}\right|^{2}\right) d s d t-M
$$

see (3.5)-(3.6). We define the mapping $F: X_{1} \rightarrow X_{2}$ as in the proof of Theorem 6.2. Analogous to the proof of Theorem 6.2, we can verify that $F^{\prime}(\mathbf{0})$ is an epimorphism, and therefore the image of $F^{\prime}(\mathbf{0})$ is closed. Hence, we have verified all the assumptions in Theorem 6.1 and we conclude that there exist a $\mathbf{q} \in X_{2}^{*}=L^{2}\left(0, T ; \mathbf{V}_{0}^{1}(\Omega)\right)$ and a $\left(\lambda_{0}, \lambda\right) \in \mathbb{R}^{2}$ such that $\left(\mathbf{q}, \lambda_{0}, \lambda\right) \neq(\mathbf{0}, 0,0)$

$$
\begin{gathered}
\left.\left\langle\mathcal{L}_{\mathbf{y}}(\mathbf{y}, \mathbf{q}), \mathbf{h}\right\rangle\right|_{\mathbf{y}=0}=0 \quad \forall \mathbf{h} \in Y, \\
\lambda_{0} \geq 0, \quad \lambda \geq 0,
\end{gathered}
$$

and

$$
\lambda\left(\int_{0}^{T} \int_{\partial \Omega}\left(\left|\partial_{t} \mathbf{w}\right|^{2}+\left|\mathbf{w}+\mathbf{v}_{\infty}\right|^{k}\right) d s d t-M\right)=0 .
$$

As in Theorems 6.2 and 6.8, we derive from (6.73) relations (6.33)-(6.34), (6.36)(6.37), (6.39)-(6.44),

$$
-\partial_{t} \mathbf{q}-\mu \Delta \mathbf{q}+\mathbf{q} \cdot(\nabla \mathbf{w})^{T}-(\mathbf{w} \cdot \nabla) \mathbf{q}-\left(\mathbf{v}_{\infty} \cdot \nabla\right) \mathbf{q}+\nabla r=\lambda_{0}(\mu \Delta \mathbf{w}-\nabla p)
$$


and

$$
\mathbf{q}(T, \cdot)+\frac{\lambda_{0}}{2} \mathbf{w}_{\sigma}(T, \cdot)=0 \quad \text { in } \mathbf{V}_{0}^{0}(\Omega)
$$

where $\mathbf{w}_{\sigma}(T, \cdot)$ is the projection of $\mathbf{w}(T, \cdot)$ onto $\mathbf{V}_{0}^{0}(\Omega)$. Moreover, we derive the following boundary condition:

$$
2 \lambda \partial_{t t}^{2} \gamma \mathbf{w}-\widetilde{\mathcal{A}}(\mathbf{w})-\mathcal{T}(\mathbf{w}, p) \mathbf{n}-\mathcal{T}(\mathbf{q}, r) \mathbf{n}=\widetilde{\eta}(t) \mathbf{n},
$$

where

$$
\widetilde{\mathcal{A}}(\mathbf{w})=\gamma\left\{k \lambda\left|\mathbf{w}+\mathbf{v}_{\infty}\right|^{k-2}\left(\mathbf{w}+\mathbf{v}_{\infty}\right)+\lambda_{0}\left(\frac{1}{2}\left(\left(\mathbf{w}+\mathbf{v}_{\infty}\right) \cdot \mathbf{n}\right) \mathbf{w}+\frac{|\mathbf{w}|^{2}}{4} \mathbf{n}\right)\right\}
$$

and

$$
\widetilde{\eta}(t)=-\int_{\partial \Omega} \widetilde{\mathcal{A}}(\mathbf{w}) \cdot \mathbf{n} d s / \int_{\partial \Omega} d s
$$

Furthermore, the following compatibility conditions hold:

$$
\begin{gathered}
{\left.[(\gamma \mathbf{w}) \cdot \mathbf{n}]\right|_{t=0}=\left(\gamma \mathbf{w}_{0}\right) \cdot \mathbf{n},} \\
\left.\left(\frac{1}{2} \lambda_{0} \gamma w_{\pi}+2 \lambda \partial_{t} \gamma \mathbf{w} \cdot \mathbf{n}\right)\right|_{t=T}=0,\left.\quad\left(\lambda \partial_{t} \gamma \mathbf{w}\right) \cdot \boldsymbol{\tau}\right|_{t=T}=0
\end{gathered}
$$

where $w_{\pi}$ is the primitive for the projection $\nabla w_{\sigma}$ of $\mathbf{w}$ onto $\nabla H_{\pi}$ determined by (6.51).

To complete the proof, it remains to show that $\lambda_{0} \neq 0$ so that we can choose $\lambda_{0}=1$. Assume $\lambda_{0}=0$. Then (6.76), (6.77), (6.36), and (6.39) yield $\mathbf{q} \equiv \mathbf{0}$ by standard techniques of energy estimates and the Gronwall inequality. Also, equation (6.76) and the condition $\int_{\partial \Omega} r d s=0$ imply $r=0$. We note that $\lambda \neq 0$ because $\left(\mathbf{q}, \lambda_{0}, \lambda, r\right)=(\mathbf{0}, 0, \lambda, 0) \neq(\mathbf{0}, 0,0,0)$. By $(6.70), \lambda>0$. Then, by virtue of $(6.71), \mathbf{w}$ is also a solution of the following modified minimization problem: seek a $\mathbf{w} \in Y$ such that the functional (3.5) is minimized subject to the equality constraints (3.1), (3.3), and

$$
\int_{0}^{T} \int_{\partial \Omega}\left(\left|\mathbf{w}+\mathbf{v}_{\infty}\right|^{k}+\left|\partial_{t} \mathbf{w}\right|^{2}\right) d s d t=M
$$

We now show that this minimization problem satisfies the conditions of Theorem 6.1. Indeed, we set $X_{1}=Y_{0}$ and $X_{2}=L^{2}\left(0, T ; \mathbf{V}^{-1}(\Omega)\right) \times \mathbb{R}$. We define the mapping $f$ by $f(\mathbf{y})=\mathcal{J}(\mathbf{w}+\mathbf{y})$, where $\mathcal{J}$ is the functional (3.5) and define

$$
\widetilde{F}(\mathbf{y})=\left(\begin{array}{c}
\partial_{t}(\mathbf{w}+\mathbf{y})-\mu P \Delta(\mathbf{w}+\mathbf{y})+P\left[\left(\left(\mathbf{w}+\mathbf{y}+\mathbf{v}_{\infty}\right) \cdot \nabla\right)(\mathbf{w}+\mathbf{y})\right] \\
\int_{0}^{T} \int_{\partial \Omega}\left(\left|\mathbf{w}+\mathbf{y}+\mathbf{v}_{\infty}\right|^{k}+\left|\partial_{t} \mathbf{w}+\partial_{t} \mathbf{y}\right|^{2}\right) d s d t-M
\end{array}\right)
$$

where $P: \mathbf{H}^{-1}(\Omega) \rightarrow \mathbf{V}^{-1}(\Omega)$ is the orthogonal projection. Then $\widetilde{F}^{\prime}(\mathbf{0}): X_{1} \rightarrow X_{2}$ is defined by

$$
\left\langle\widetilde{F}^{\prime}(\mathbf{0}), \mathbf{y}\right\rangle=\left(\begin{array}{c}
\partial_{t} \mathbf{y}-\mu P \Delta \mathbf{y}+P(\mathbf{y} \cdot \nabla) \mathbf{w}+P\left(\left(\mathbf{w}+\mathbf{v}_{\infty}\right) \cdot \nabla\right) \mathbf{y} \\
\int_{0}^{T} \int_{\partial \Omega}\left(k\left|\mathbf{w}+\mathbf{v}_{\infty}\right|^{k-2}\left(\mathbf{w}+\mathbf{v}_{\infty}\right) \cdot \mathbf{y}+2 \partial_{t} \mathbf{w} \cdot \partial_{t} \mathbf{y}\right) d s d t
\end{array}\right) .
$$


To show that $\widetilde{F}^{\prime}(\mathbf{0})$ is an epimorphism, we first observe that this operator is continuous. Next we need to show that for each $\mathbf{f} \in L^{2}\left(0, T ; \mathbf{V}^{-1}(\Omega)\right)$ and $\zeta \in \mathbb{R}$, the system

$$
\begin{gathered}
\left\langle\partial_{t} \mathbf{y}(t), \mathbf{z}\right\rangle+\mu \int_{\Omega} \nabla \mathbf{y}(t): \nabla \mathbf{z} d \mathbf{x}+\int_{\Omega}\left(\left(\mathbf{w}(t)+\mathbf{v}_{\infty}\right) \cdot \nabla\right) \mathbf{y}(t) \cdot \mathbf{z} d \mathbf{x} \\
+\int_{\Omega}(\mathbf{y}(t) \cdot \nabla) \mathbf{w}(t) \cdot \mathbf{z} d \mathbf{x}=\int_{\Omega} \mathbf{f}(t) \cdot \mathbf{z} d \mathbf{x} \quad \forall \mathbf{z} \in \mathbf{V}_{0}^{1}(\Omega), \text { a.e. } t \in(0, T), \\
\left.\mathbf{y}\right|_{t=0}=\mathbf{0} \quad \text { in } \mathbf{V}^{0}(\Omega),
\end{gathered}
$$

and

$$
\int_{0}^{T} \int_{\partial \Omega}\left(k\left|\mathbf{w}+\mathbf{v}_{\infty}\right|^{k-2}\left(\mathbf{w}+\mathbf{v}_{\infty}\right) \cdot \mathbf{y}+2 \partial_{t} \mathbf{w} \cdot \partial_{t} \mathbf{y}\right) d s d t=\zeta
$$

has a solution $\mathbf{y} \in Y_{0}$.

To this end we first look for a $\mathbf{y} \in \gamma_{S_{T}} Y_{0}$ satisfying (6.83). $\left(\gamma_{S_{T}} Y_{0}\right.$ is the space of functions belonging to $Y_{0}$ restricted to $S_{T}$.) It suffices to show that there exists a $\mathbf{y} \in \gamma_{S_{T}} Y_{0}$ for which the left side of (6.83) is not zero, for then we can obtain (6.83) by multiplying $\mathbf{y}$ by a suitable constant. Suppose that for every $\mathbf{y} \in \gamma_{S_{T}} Y_{0}$ the equality

$$
\int_{0}^{T} \int_{\partial \Omega}\left(k\left|\mathbf{w}+\mathbf{v}_{\infty}\right|^{k-2}\left(\mathbf{w}+\mathbf{v}_{\infty}\right) \cdot \mathbf{y}+2 \partial_{t}\left(\mathbf{w}+\mathbf{v}_{\infty}\right) \cdot \partial_{t} \mathbf{y}\right) d s d t=0
$$

holds. This equality and (6.79) with $\lambda_{0}=0$ imply that $\mathbf{w}+\mathbf{v}_{\infty}$ satisfies the relations

$$
-2 \partial_{t t}\left(\mathbf{w}+\mathbf{v}_{\infty}\right)+k\left|\mathbf{w}+\mathbf{v}_{\infty}\right|^{k-2}\left(\mathbf{w}+\mathbf{v}_{\infty}\right)=\mathbf{0} \quad \text { on }(0, T) \times \partial \Omega
$$

and

$$
\left.\partial_{t}\left(\mathbf{w}+\mathbf{v}_{\infty}\right)\right|_{t=T}=\mathbf{0} \quad \text { on } \partial \Omega
$$

Note also that

$$
\left.\left(\mathbf{w}+\mathbf{v}_{\infty}\right)\right|_{t=0, \mathbf{x} \in \partial \Omega}=\left.\mathbf{v}_{0}\right|_{\partial \Omega} \equiv \mathbf{0}
$$

We multiply $(6.84)$ by $\partial_{t}\left(\mathbf{w}+\mathbf{v}_{\infty}\right)$ and obtain

$$
-\partial_{t}\left|\partial_{t}\left(\mathbf{w}+\mathbf{v}_{\infty}\right)\right|^{2}+\partial_{t}\left|\mathbf{w}+\mathbf{v}_{\infty}\right|^{k}=0 \quad \text { on }(0, T) \times \partial \Omega,
$$

which together with (6.85) implies

$$
-\left|\partial_{t}\left(\mathbf{w}+\mathbf{v}_{\infty}\right)\right|^{2}+\left|\mathbf{w}+\mathbf{v}_{\infty}\right|^{k}=\left|\mathbf{w}(T, \cdot)+\mathbf{v}_{\infty}\right|^{k} \quad \text { on }(0, T) \times \partial \Omega
$$

This equality taken at $t=0$ yields

$$
-\left|\partial_{t}\left(\mathbf{w}(0, \mathbf{x})+\mathbf{v}_{\infty}\right)\right|^{2}=\left|\mathbf{w}(T, \mathbf{x})+\mathbf{v}_{\infty}\right|^{k} \quad \text { on } \partial \Omega
$$

which implies

$$
\left|\partial_{t}\left(\mathbf{w}(0, \mathbf{x})+\mathbf{v}_{\infty}\right)\right|^{2}=0 \quad \text { and } \quad\left|\mathbf{w}(T, \mathbf{x})+\mathbf{v}_{\infty}\right|^{k}=0 \quad \text { on } \partial \Omega
$$


or we rewrite the last relation as

$$
\mathbf{w}(T, \mathbf{x})+\mathbf{v}_{\infty}=\mathbf{0} \quad \text { on }(0, T) \times \partial \Omega .
$$

We deduce from the differential equation (6.84) and boundary conditions (6.85) and (6.87) that $\left(\mathbf{w}+\mathbf{v}_{\infty}\right)=\mathbf{0}$ on $(0, T) \times \partial \Omega$. This contradicts (6.80). Therefore, there exists a $\mathbf{z} \in \gamma_{S_{T}} Y_{0}$ satisfying (6.83), where $\mathbf{y}$ is replaced by $\mathbf{z}$.

We supplement the system (6.81)-(6.82) with the boundary condition

$$
\left.\mathbf{y}\right|_{(0, T) \times \partial \Omega}=\mathbf{z} .
$$

Using the techniques in the proof of Theorem 4.4 we see that (6.81)-(6.82) and (6.88) indeed has a (unique) solution $\mathbf{y} \in \mathcal{V}^{(1)}\left(Q_{T}\right)$ (the situation now is even simpler, as the system (6.81)-(6.82) is linear). Note that substituting $\mathbf{z}$ from (6.88) into (6.83) in place of $\mathbf{y}$ makes $(6.83)$ valid. Clearly, $\mathbf{y} \in Y_{0}$. Hence we have proved that $\widetilde{F}^{\prime}(0)$ is an epimorphism, so that we have verified all the assumptions in Theorem 6.1. By virtue of Theorem 6.1, every Lagrange multiplier triplet $\left(\widetilde{\mathbf{q}}, \widetilde{\lambda}_{0}, \widetilde{\lambda}\right)$ such that $(6.73)$ holds where $\mathcal{L}$ is defined by $(6.72)$ satisfies $\widetilde{\lambda}_{0} \neq 0$; in particular, $\left(\mathbf{q}, \lambda_{0}, \lambda\right)$ is such a triplet, and therefore $\lambda_{0} \neq 0$. This contradicts the assumption $\lambda_{0}=0$. Hence $\lambda_{0} \neq 0$.

REMARK. Note that, since we have not employed a separate variable for the control, the boundary condition (3.2) does not appear in the optimality systems of sections 6.3-6.5. In fact, in order to satisfy (3.2) one merely has to choose, once $\mathbf{w}$ is determined from the above optimality system, a control $\mathbf{g}$ such that $\mathbf{g}=\left.\mathbf{w}\right|_{\partial \Omega}+\mathbf{v}_{\infty}$ for $t \in(0, T)$.

REMARK. The complexity of the optimality systems makes it nontrivial to study the regularity of solutions for these systems. The regularity of solutions will be studied elsewhere.

\section{REFERENCES}

[1] R. Adams, Sobolev Spaces, Academic Press, New York, 1975.

[2] F. Abergel and R. Temam, On some control problems in fluid mechanics, Theoret. Comput. Fluid Dynamics, 1 (1990) pp. 303-325.

[3] V. Alekseev, V. Tikhomirov, And S. Fomin, Optimal Control, Consultants Bureau, New York, 1987.

[4] O. Besov, V. IL'in, And S. Nikol'skiI, Integral Representations of Functions and Imbedding Theorems, Winston, Washington, D.C., 1979.

[5] P. Constantin And C. Foias, Navier-Stokes Equations, Univ. of Chicago Press, Chicago, IL, 1988.

[6] C. Cuverlier, Optimal control of a system governed by the Navier-Stokes equations coupled with the heat equation, in New Developments in Differential Equations, W. Eckhaus, ed., North-Holland, Amsterdam, 1976, pp. 81-98.

[7] H. Fattorini And S. SRitharan, Existence of optimal controls for viscous flow problems, Proc. Roy. Soc. London Ser. A, 439 (1992), pp. 81-102.

[8] H. Fattorini and S. SRitharan, Necessary and sufficient conditions for optimal controls in viscous flow problems, Proc. Roy. Soc. London Ser. A, 124 (1994), pp. 211-251.

[9] H. Fattorini and S. SRitharan, Optimal chattering control for viscous flows, Nonlinear Anal., 25 (1995), pp. 763-797.

[10] H. FAtTORIni AND S. SRIthaRAn, Optimal control theory for viscous flow problems, to appear.

[11] A. FuRsikov, On some control problems and results concerning the unique solvability of a mixed boundary value problem for the three-dimensional Navier-Stokes and Euler systems, Soviet Math. Dokl., 21 (1980), pp. 889-893.

[12] A. Fursikov, Control problems and theorems concerning the unique solvability of a mixed boundary value problem for the three-dimensional Navier-Stokes and Euler equations, Math USSR Sb., 43 (1982), pp. 281-307. 
[13] A. Fursikov, Properties of solutions of some extremal problems connected with the NavierStokes system, Math USSR Sb., 46 (1983), pp. 323-351.

[14] V. Girault And P.-A. Raviart, Finite Element Methods for Navier-Stokes Equations, Springer-Verlag, Berlin, 1986.

[15] M. Gunzburger, L. Hou, And T. Svobodny, Numerical approximation of an optimal control problem associated with the Navier-Stokes equations, Appl. Math. Lett., 2 (1989), pp. 2931.

[16] M. Gunzburger, L. Hou, And T. Svobodny, Analysis and finite element approximation of optimal control problems for the stationary Navier-Stokes equations with Dirichlet controls, Modél. Math. Anal. Numér., 25 (1991), pp. 711-748.

[17] M. Gunzburger, L. Hou, And T. Svobodny, Analysis and finite element approximation of optimal control problems for the stationary Navier-Stokes equations with distributed and Neumann controls, Math. Comp., 57 (1991), pp. 123-151.

[18] M. Gunzburger, L. Hou, And T. Svobodny, Boundary velocity control of incompressible flow with an application to viscous drag reduction, SIAM J. Control Optim., 30 (1992), pp. $167-181$

[19] O. Ladyzhenskaya, The Mathematical Theory of Viscous Incompressible Flow, Gordon and Breach, New York, 1963.

[20] J.-L. Lions, Control of Distributed Singular systems, Bordas, Paris, 1985.

[21] J.-L. Lions And E. Magenes, Non-Homogeneous Boundary Value Problems and Applications I, Springer-Verlag, Berlin, 1972.

[22] P. Panagiotopoulos, Inequality Problems in Mechanics and Applications, Birkhäuser, Boston, 1985.

[23] J. Serrin, Mathematical principles of classical fluid mechanics, in Handbuch der Physik, VIII/1, S. Flügge and C. Truesdell, eds., 1959, pp. 1-125.

[24] S. Sritharan, An optimal control problem in exterior hydrodynamics, in Distributed Parameter Control Systems, New Trends and Applications, G. Chen, B. Lee, W. Littman, and L. Markus, eds., Marcel Dekker, New York, 1991, pp. 385-417.

[25] S. SRItharan, Dynamic programming of the Navier-Stokes equations, System Control Lett., 16 (1991), pp. 299-307.

[26] S. SRitharan, An optimal control problem in exterior hydrodynamics, Roy. Soc. Edinburgh Proc. A, 121 (1992), pp. 5-32.

[27] S. Sritharan, On Hamilton-Jacobi equation in infinite dimensions, in Nonlinear Problems in Aviation and Aerospace, S. Sivasundaram, ed., Embry-Riddle University Press, Daytona Beach, FL, 1994, pp. 631-638.

[28] R. Temam, Navier-Stokes Equations, North-Holland, Amsterdam, 1979.

[29] M. Triebel, Interpolation Theory, Function Spaces, Differential Operators, Veb Deutscher Verlag der Wissenschaften, Berlin, 1978; also, North-Holland, Amsterdam, New York, 1978 . 